\title{
SIMULATED GROUND-WATER FLOW, NAVAL AIR WARFARE CENTER, WEST TRENTON, NEW JERSEY
}

Water-Resources Investigations Report 02-4019

Prepared in cooperation with the U.S. DEPARTMENT OF THE NAVY

\section{₹USGS}




\section{Errata}

Cases where the stated model input parameters are not those actually used in the model to obtain the results stated in the report:

Page 17, table 2, column entitled "Horizontal hydraulic conductivity used in model":

- For the Lockatong Formation, rocks 26-50 ft below land surface: $9.4 \mathrm{ft} / \mathrm{d}$ (not $11.3 \mathrm{ft} / \mathrm{d}$ ) was used in the model.

- For the Lockatong Formation, rocks 51-100 ft below land surface: $6.9 \mathrm{ft} / \mathrm{d}$ (not $4.0 \mathrm{ft} / \mathrm{d}$ ) was used in the model.

Page 19, last paragraph in "Vertical Hydraulic Conductivity" section, last sentence should read:

- The best model calibration was achieved when the vertical hydraulic conductivity of the bed of the Delaware River was set at $0.005 \mathrm{ft} / \mathrm{d}$, the vertical conductivity of the bed of all other streams was set at $0.05 \mathrm{ft} / \mathrm{d}$, the thickness of the riverbed was set at $1 \mathrm{ft}$, and the thickness of the beds of the tributaries was set at $0.1 \mathrm{ft}$.

Cases where the stated model output was typed incorrectly:

- Page 24, Table 4, the simulated water level at well $36 \mathrm{~S}$ is $\mathbf{1 5 2 . 1 0} \mathbf{f t}$, (not 151.10 f).

- Page 36, Table 10:

- In Simulation 2 (recovery-well network 2), a pathline originating at well 49BR discharges to the west branch of Gold Run (not to well $48 \mathrm{BR}$ ).

o In Simulation 1 (with no pumpage at the NAWC), a pathline originating at well 51BR discharges to the main stem of Gold Run (not to the Delaware River). 


\section{SIMULATED GROUND-WATER FLOW, NAVAL AIR WARFARE CENTER, WEST TRENTON, NEW JERSEY}

By Jean C. Lewis-Brown and Donald E. Rice

U.S. GEOLOGICAL SURVEY

Water-Resources Investigations Report 02-4019

Prepared in cooperation with the

U.S. DEPARTMENT OF THE NAVY

West Trenton, New Jersey

2002

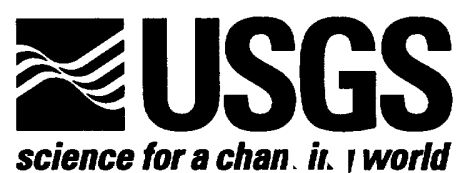




\title{
U.S. DEPARTMENT OF THE INTERIOR \\ GALE A. NORTON, Secretary
}

\author{
U.S. GEOLOGICAL SURVEY \\ Charles G. Groat, Director
}

The use of firm, trade, and brand names in the report is for identification purposes only and does not constitute endorsement by the U.S. Government.

For additional information write to:

District Chief

U.S. Geological Survey Mountain View Office Park 810 Bear Tavern Road West Trenton, NJ 08628
Copies of this report can be purchased from:

U.S. Geological Survey Branch of Information Services Box 25286

Denver, CO 80225-0286 


\section{CONTENTS}

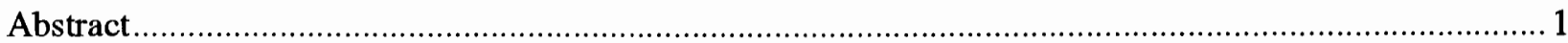

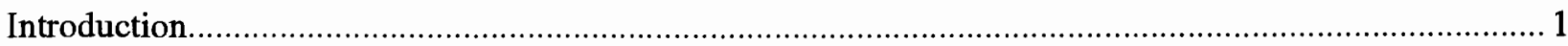

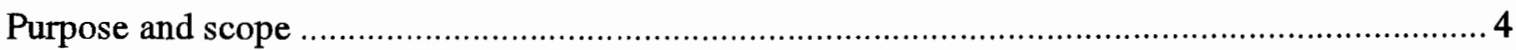

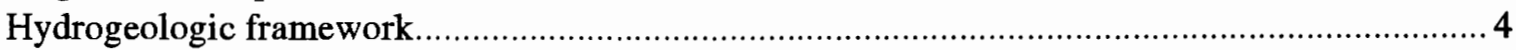

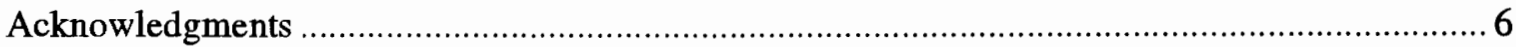

Description of ground-water-flow model ……………

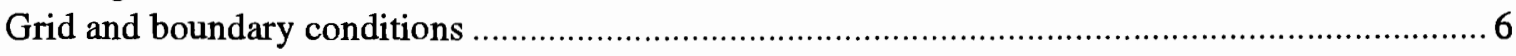

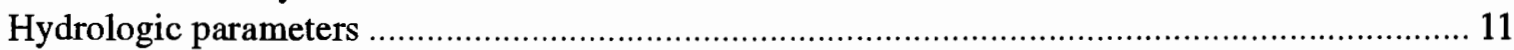

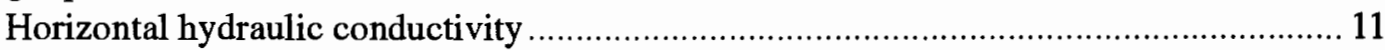

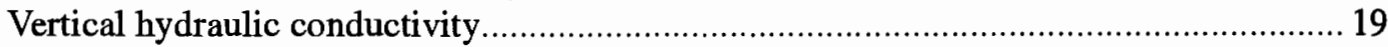

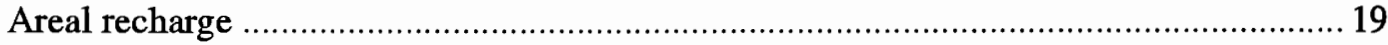

Specific yield and storage coefficient....................................................................... 19

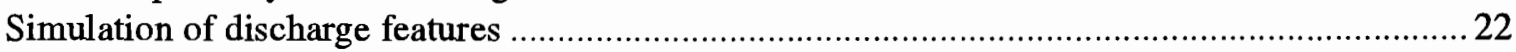

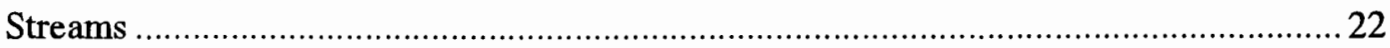

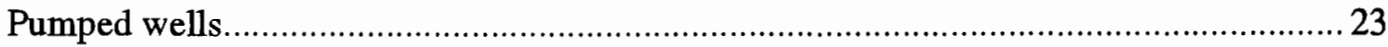

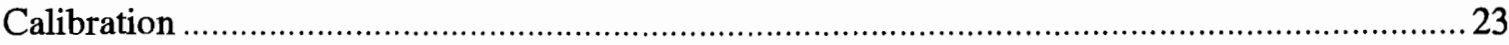

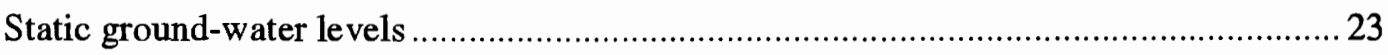

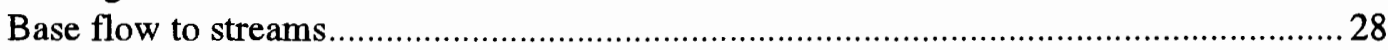

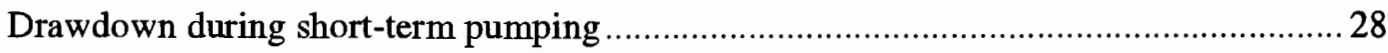

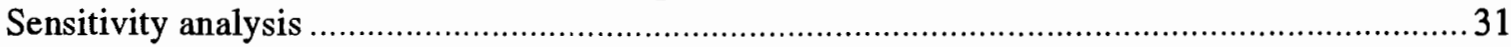

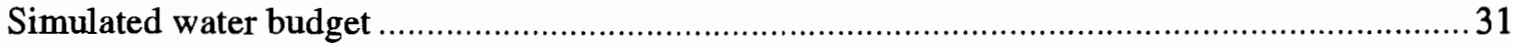

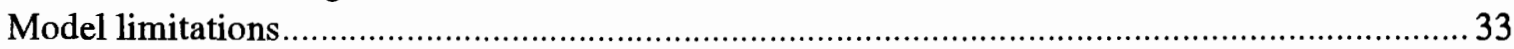

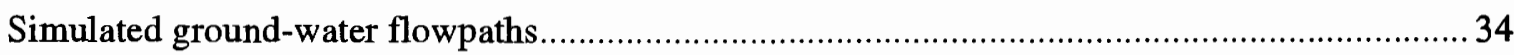

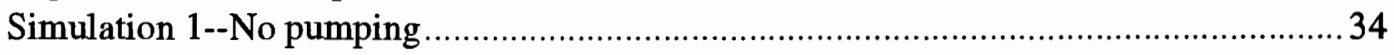

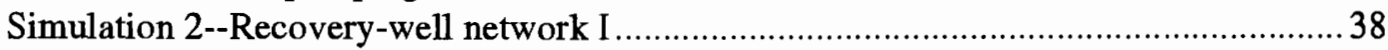

Simulation 3--Recovery-well network II.................................................................... 38

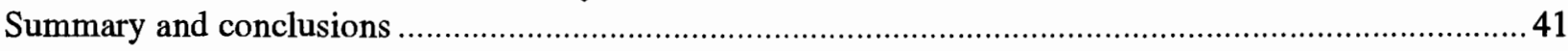

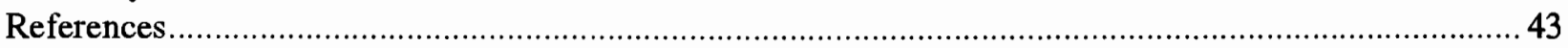

\section{ILLUSTRATIONS}

Figure 1. Map showing location of the Naval Air Warfare Center and vicinity, Ewing Township, New Jersey, and extent of model grid.............................................................................

2. Map showing well locations and line of section A-A' at the Naval Air Warfare Center, West

Trenton, New Jersey .........................................................................................

3 Geologic section A-A' showing bedding units and fault zone at the Naval Air Warfare Center, West Trenton, New Jersey ………………………......................................5

4 Map showing finite-difference grid for the ground-water-flow model, Naval Air Warfare Center, West Trenton, New Jersey, and vicinity....................................................... 7

5 Map showing part of the ground-water-flow model grid showing cells used to simulate Gold Run and the fault in the area of the Naval Air Warfare Center, West Trenton, New Jersey.

6. Section showing vertical discretization of ground-water-flow model along geologic section A-A' at the Naval Air Warfare Center, West Trenton, New Jersey. 


\section{ILLUSTRATIONS--Continued}

Page

Figure 7. Graphs showing sensitivity of simulated ground-water levels, base flow, drawdown, and capture of contaminated water by recovery wells to variations in the values of motel parameters, Naval Air Warfare Center, West Trenton, New Jersey

8. Map showing simulated discharge locations of ground water at and near contaminated wells at the Naval Air Warfare Center, West Trenton, New Jersey (no recovery wells simulated as pumping) .37

9. Schematic section showing generalized ground-water-flow path 39

10. Map showing simulated discharge locations of ground water at and near contaminated wells at the Naval Air Warfare Center, West Trenton, New Jersey (Recovery-Well Network

I or II in operation)

\section{TABLES}

Table 1. Horizontal hydraulic conductivity at, and well construction data for, wells at and near the Naval Air Warfare Center, West Trenton, New Jersey

2. Horizontal hydraulic conductivity as a function of depth below land surface, Naval Air

Warfare Center, West Trenton, New Jersey 17

3. Base flow and ground-water runoff in streams at or near the Naval Air Warfare Center, West Trenton, New Jersey....

4. Measured and simulated water levels in wells at and near the Naval Air Warfare Center, West

Trenton, New Jersey, May 18 and 26, 2000

5. Measured and simulated base flow to streams at and near the Naval Air Warfare Center, West

Trenton, New Jersey .28

6. Measured and simulated drawdown in wells at the Naval Air Warfare Center, West Trenton, New Jersey, after well 15BR had been pumped at 25 gallons per minute for 72 hour: .... 29

7. Measured and simulated drawdown in wells at the Naval Air Warfare Center, West Trenton, New Jersey, after well BRP1 had been pumped at 12.5 gallons per minute for 48 ho'urs. 30

8. Measured and simulated drawdown in wells at the Naval Air Warfare Center, West Trenton, New Jersey, after well 5BR had been pumped at 25 gallons per minute for 42 hours..... 30

9. Simulated water budget at and near the Naval Air Warfare Center, West Trenton, New Jersey

10. Simulated discharge points of ground water at and near contaminated wells at the Naval Air Warfare Center, West Trenton, New Jersey. 


\section{CONVERSION FACTORS, AND VERTICAL DATUM}

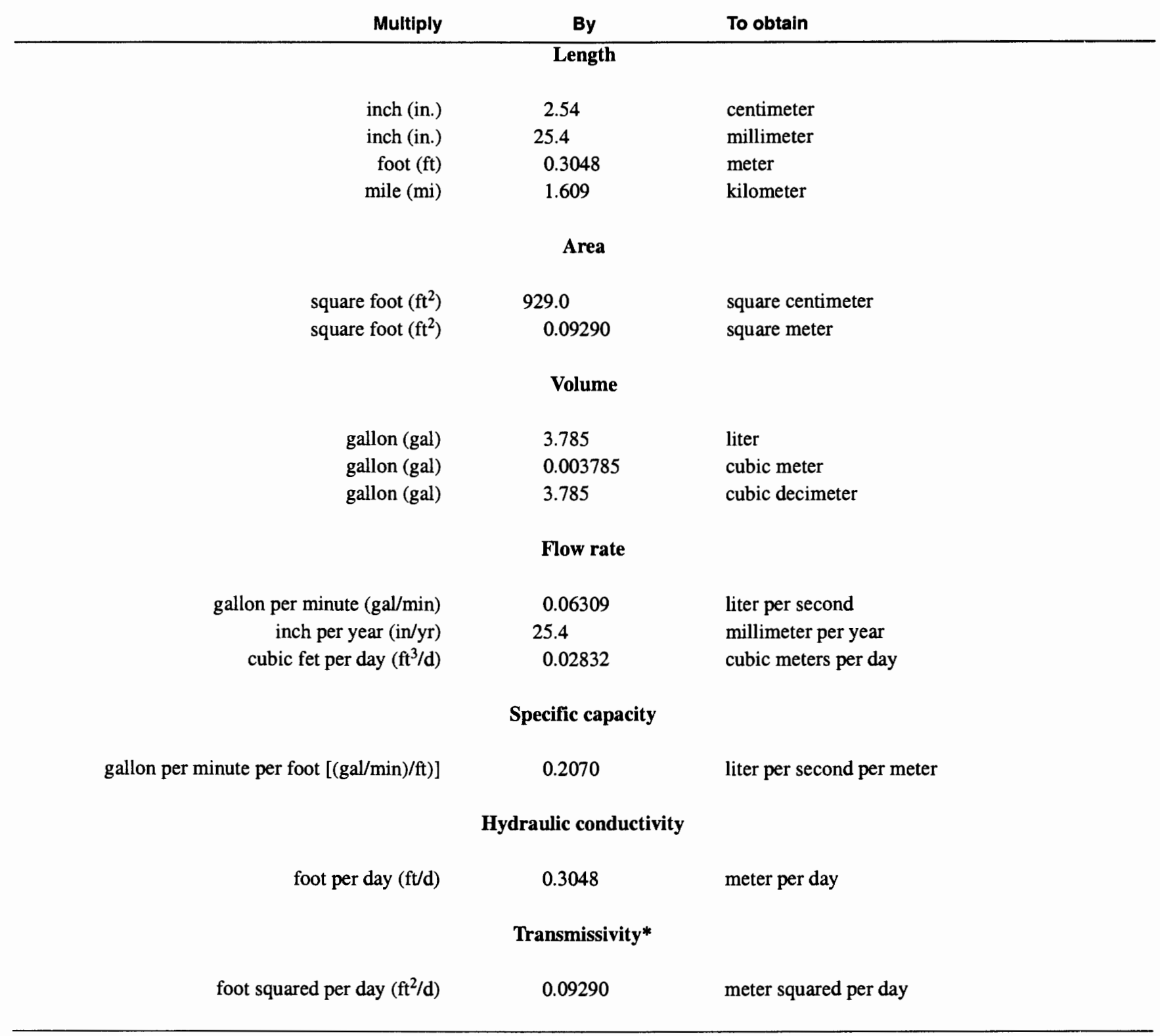

Sea Level: In this report, "sea level" refers to the National Geodetic Vertical Datum of 1929 (NGVD of 1929) - a geodetic datum derived from a general adjustment of the first-order level nets of both the United States and Canada, formerly called Sea Level Datum of 1929.

Altitude: In this report, "altitude" refers to distance above or below the National Geodetic Vertical datum of 1929 (NGVD of 1929)

Latitude, Longitude: In this report, latitude and longitude are referenced to North American Datum of 1927 (NAD of 1927)

*Transmissivity: The standard unit for transmissivity is cubic foot per day per square foot times foot of aquifer thickness $\left[\left(\mathrm{ft}^{3} / \mathrm{d}\right) / \mathrm{ft}^{2}\right] \mathrm{ft}$. In this report, the mathematically reduced form, foot squared per day ( $\left.\mathrm{ft}^{2} / \mathrm{d}\right)$, is used for convenience. 


\title{
Simulated Ground-Water Flow, Naval Air Warfare Center, West Trenton, New Jersey
}

\author{
by Jean C. Lewis-Brown and Donald E. Rice
}

\section{ABSTRACT}

The effectiveness of ground-water pumping by the use of recovery wells in preventing contaminated ground water at the Naval Air Warfare Center (NAWC), West Trenton, N.J., from moving off the NAWC property was evaluated by use of a digital model of ground-water flow. The finite-difference, three-dimensional, porous-medium model uses the MODFLOW code to solve the groundwater-flow equation. Ground-water flowpaths from areas at and near contaminated well sites for a nopumping and two recovery-well-pumping scenarios were computed using the post-processor MODPATH.

The NAWC is underlain by the Lockatong and Stockton Formations, which consist of alternating layers of mudstone, siltstone, and sandstone. A fault transects the area and forms the contact between the Lockatong and Stockton Formations. Water flows through these rocks primarily in fractures that are parallel to the strike of the bedding-N. $65^{\circ}$ E. --and, to a lesser degree, in fractures that are nearly vertical.

The median horizontal hydraulic conductivity measured at 32 shallow wells (less than $25 \mathrm{ft}$ (feet) deep) at the NAWC is $2.6 \mathrm{ft} / \mathrm{d}$ (feet/day). At 17 wells, 26 to $50 \mathrm{ft}$ deep, the median horizontal hydraulic conductivity is $11.3 \mathrm{ft} / \mathrm{d}$. The hydraulic conductivity is lower in the shallow rocks than in the rocks from 26 to $50 \mathrm{ft}$ below land surface because the shallow rocks are weathered, and clay and silt from the weathering process fills many of the fractures. Deeper than $50 \mathrm{ft}$, the horizontal hydraulic conductivity decreases, probably because the interconnection of fractures decreases with depth. The broad range in measured hydraulic conductivity $(0.001$ to $227 \mathrm{ft} / \mathrm{d})$ is a result of the heterogeneity of the fractured-rock aquifer. Wells where high hydraulic conductivities were measured probably are completed where one or more vertical fractures intersect horizontal fractures, whereas wells with low hydraulic conductivities probably are between vertical fractures.

Under no-pumping conditions, simulated ground-water flowpaths from the NAWC are primarily in the direction of the strike of the bedding units, with most water from the NAWC flowing west-southwest to discharge at Gold Run on and adjacent to the NAWC. Under recovery-wellpumping conditions, most flowpaths are diverted toward the pumped wells.

Two different recovery-well networks, each comprising six wells pumping a total of 56.5 gallons per minute, have been used at the NAWC. Modeling results indicate that both networks capture water from the area around 51 of the 55 contaminated wells at the NAWC and that the second network captures more potentially contaminated ground water that would otherwise discharge to Gold Run.

\section{INTRODUCTION}

Volatile organic compounds, predominantly trichloroethylene (TCE) and its degradation products cis-1,2-dichloroethylene (cis-DCE) and vinyl chloride (VC), have been detected in ground water at the Naval Air Warfare Center (NAWC), West Trenton, N.J. (International Technology Corporation, 1994). The U.S. Geological Survey (USGS) provided technical oversight and assistance to the U.S. Department of the Navy to conduct a hydrogeologic investigation of the NAWC. In an earlier phase of the investigation, Lacombe $(2000,2001)$ determined the hydrogeologic framework at the NAWC. The location of the NAWC and surrounding area is shown in figure 1. A more detailed picture of the NAWC site, including the location of buildings and wells, is shown in figure 2. 


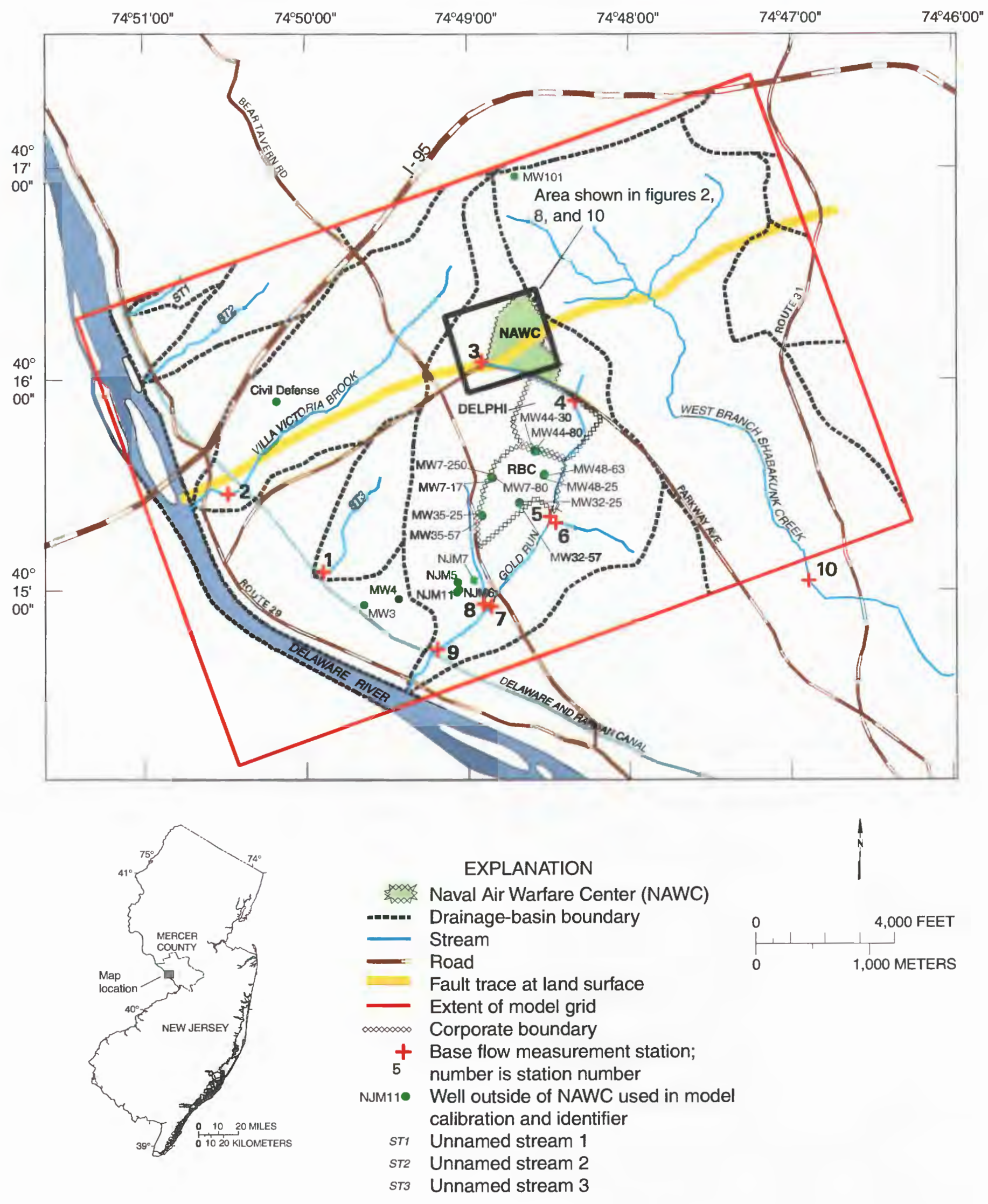

Figure 1. Location of the Naval Air Warfare Center and vicinity, Ewing Township, New Jersey, and extent of the model grid. 


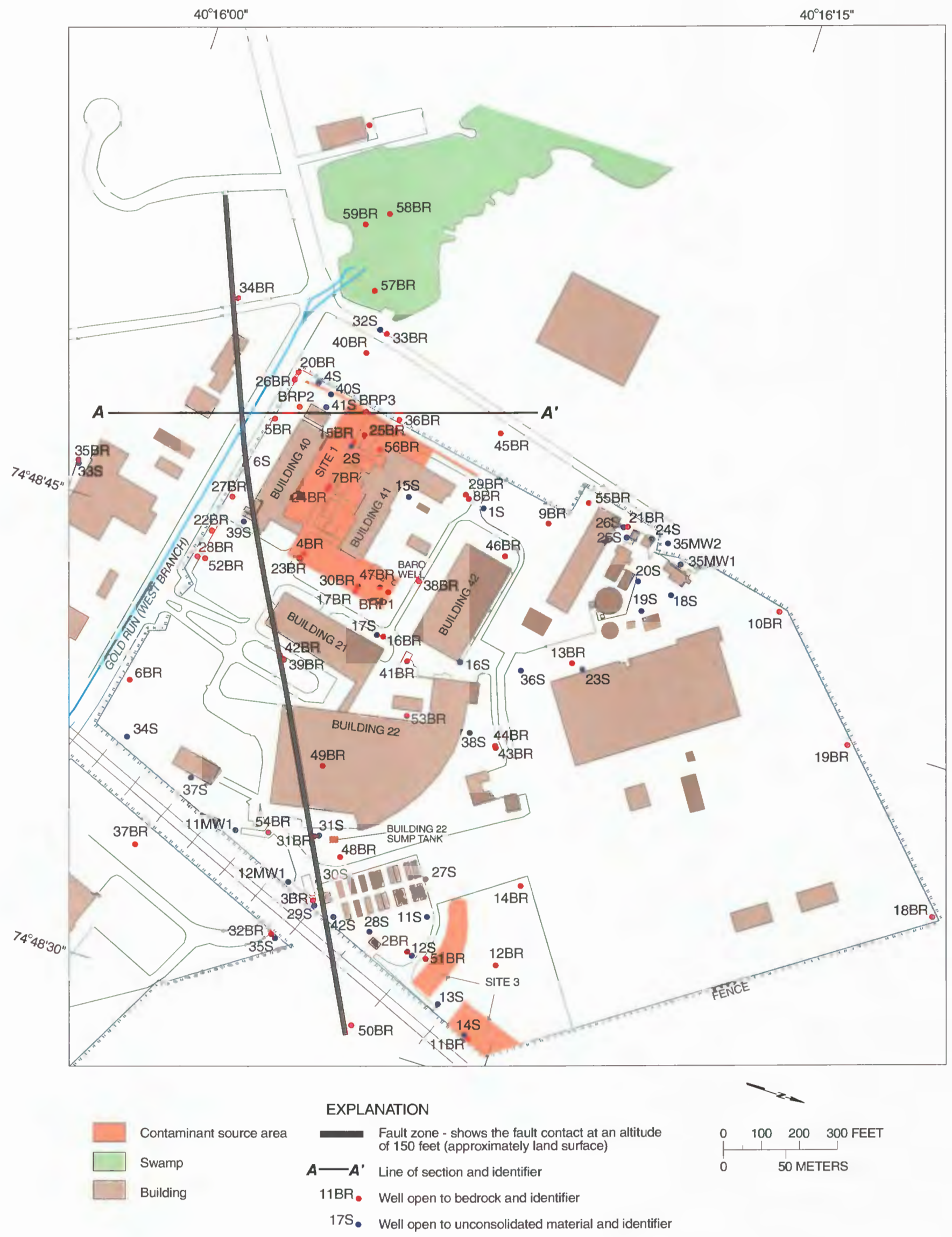

Figure 2. Well locations and line of section $A-A^{\prime}$ at the Naval Air Warfare Center, West Trenton, New Jersey. 
The phase of the investigation reported herein concerns the simulated advective pathways of these contaminants and the effectiveness of two different recovery-well networks in containing the contamination on the NAWC property. The first network (hereinafter referred to as "recovery-well Network I") was in use from January 1998 through November 2000 . The recovery wells and pumping rates used in this network were well $15 \mathrm{BR}$ at 15 $\mathrm{gal} / \mathrm{min}$, well $20 \mathrm{BR}$ at $8.5 \mathrm{gal} / \mathrm{min}$, well $22 \mathrm{BR}$ at $4.4 \mathrm{gal} / \mathrm{min}$, well $41 \mathrm{BR}$ at $10 \mathrm{gal} / \mathrm{min}$, well $45 \mathrm{BR}$ at $5.1 \mathrm{gal} / \mathrm{min}$, and well $48 \mathrm{BR}$ at $13.5 \mathrm{gal} / \mathrm{min}$. Waterlevel data collected in May 2000, after that network was in place for more than 16 months, indicated that some ground water from contaminated areas of the NAWC may have been discharging into the uppermost 500-ft reach of Gold Run (fig. 1) (Lacombe, 2001). Consequently, the network was modified in December 2000 by replacing well 41BR with well BRP2. This second network is hereinafter referred to as "recovery well network II."

\section{Purpose and Scope}

The purpose of this report is to illustrate simulated ground-water flowpaths from well locations where contaminated ground water has been detected at the NAWC. Three scenarios were simulated: no pumping at the NAWC, operation of recovery-well network I, and operation of recovery-well network II. The ground-water-flow model used to make these simulations is described, as are the limitations of using this model to simulate movement of water. Results of aquifer testing at the NAWC also are included, as are data from stream-discharge measurements at and near the NAWC.

\section{Hydrogeologic Framework}

The hydrogeologic framework of the rocks underlying the NAWC is described by Lacombe $(2000,2001)$ and is summarized here. The NAWC is underlain by layers of mudstone, siltstone, and sandstone of the Lockatong and Stockton Formations. The Lockatong Formation consists primarily of mudstone with smaller amounts of siltstone, whereas the Stockton Formation consists primarily of sandstone with smaller amounts of mudstone. The Stockton Formation has some primary porosity in rocks near land surface; the Lockatong Formation, however, has almost no primary porosity, so virtually all ground water in the Lockatong Formation is transmitted through fractures and joints (Kasabach, 1966).

Lacombe (2000) identified 20 bedding units underlying the NAWC. These bedding units generally are 20 to $60 \mathrm{ft}$ thick and are differentiated primarily on the basis of differences in lithology. Each bedding unit is composed of many waterbearing units and semiconfining units. The waterbearing units are areally extensive and have interconnected bedding-plane partings and vertical partings that produce water, whereas the semiconfining units have a limited number of partings or poorly connected partings that produce little water (Lacombe, 2000). The units strike N. $60^{\circ}$ E. to N. $70^{\circ} \mathrm{E}$. and dip 15 to $70^{\circ} \mathrm{NW}$. The steepest dip angles are in rocks near a fault that cuts through the layers in the southern part of the NAWC site, and the gentlest dip angles are in the rocks most distant from the fault. The framework is shown in section view in figure 3 .

Lacombe (2000) also described fractures that are parallel to the strike of the bedding units and two sets of near-vertical fractures. The more predominant of the vertical sets strikes N. $50^{\circ} \mathrm{E}$; the other strikes N. $20^{\circ} \mathrm{W}$. Fractures parallel to the strike of bedding units (bedding-plane fractures) are much more continuous than the vertical fractures. Bedding-plane fractures typically extend the full length of any given outcrop. Vertical fractures typically extend 100 to $200 \mathrm{ft}$ laterally but only 1 to $10 \mathrm{ft}$ vertically. Vertical fractures generally are less than $5 \mathrm{ft}$ high. The density of vertical fractures is about one fracture per $2 \mathrm{ft}$ in sandstone and five fractures per $1 \mathrm{ft}$ in mudstone and siltstone (Lacombe, 2000).

The fault that cuts through the NAWC property is at or near the contact between the Stockton and Lockatong Formations. The location of the fault initially was described by Lacombe (2000). On the basis of additional data, the interpreted location of the eastern part of the fault was moved slightly to the south (Lacombe, 2001). The 


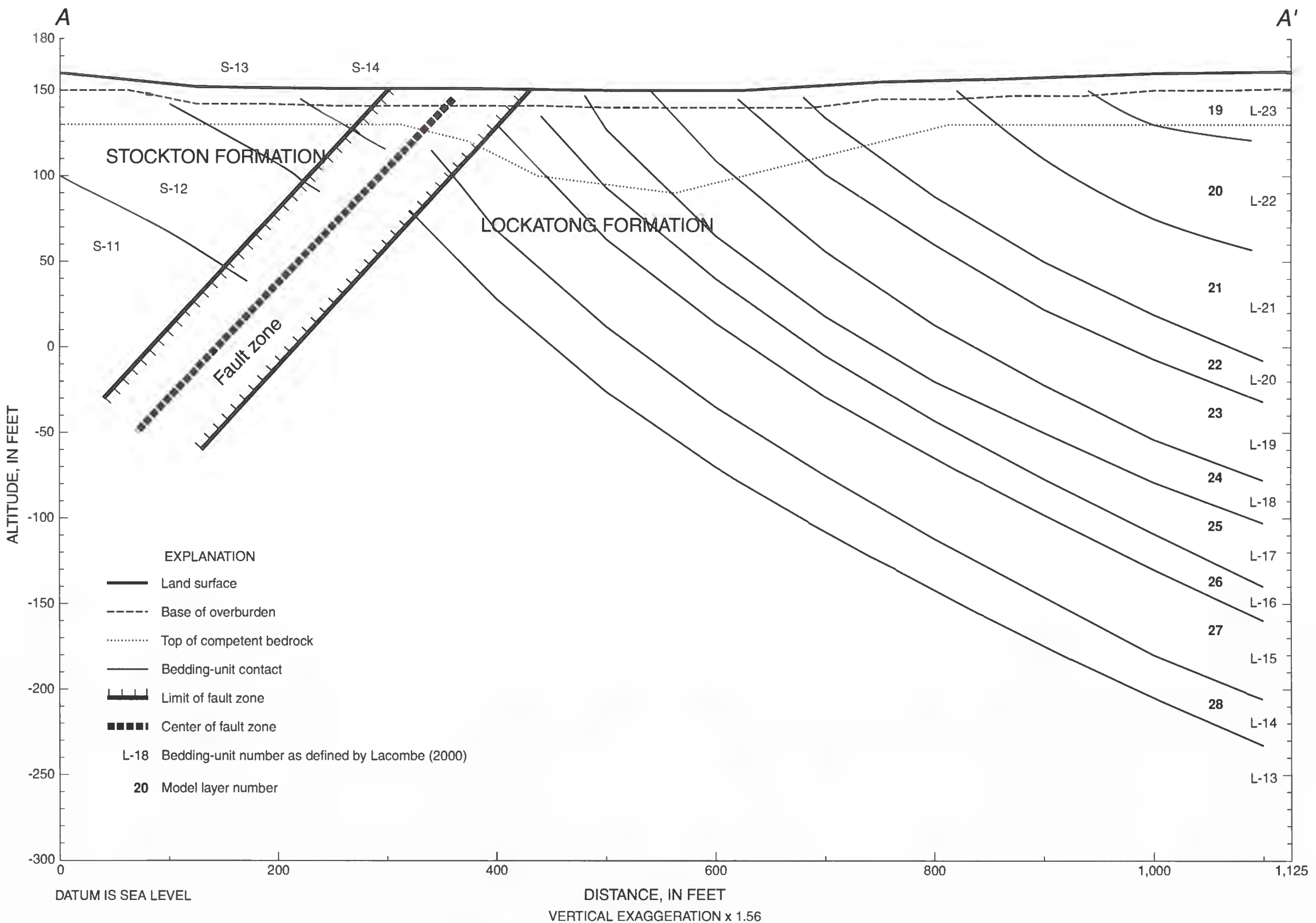

Figure 3. Geologic section $A-A^{\prime}$ showing bedding units and fault zone at the Naval Air Warfare Center, West Trenton, New Jersey. (Line of section in figs. 2 and 5. Modified from Lacombe, 2000, section $H-H^{\prime}$, fig. 17). 
fault strikes about $\mathrm{N} .70^{\circ} \mathrm{E}$. and dips about $40^{\circ} \mathrm{SE}$. In rock cores collected from the fault zone, the rocks are weathered extensively and fractured, and the fractures are completely filled with clay. Dry clay as much as $60 \mathrm{ft}$ thick was found in rock cores from some parts of the fault zone. Abundant clay in the fault zone and the absence of drawdown in wells on the south side of the fault when wells on the north side were pumped indicate that the fault is a boundary for ground-water flow (Lacombe, 2000).

Near land surface, the rocks are weathered. At depths up to about $10 \mathrm{ft}$ below land surface, the rocks have weathered completely into unconsolidated clay, silt, and sand. Beneath the unconsolidated deposits, the rocks are highly weathered and fractured, and many of the fractures are filled with clay and silt derived from the weathering process. This weathered zone extends about 15 to $60 \mathrm{ft}$ below land surface. Beneath these weathered rocks are rocks that appear to be nearly unweathered. These unweathered rocks are less fractured than the overlying rocks. Fractures in the unweathered zone are not filled with weathering products, but some are filled with precipitates.

\section{Acknowledgments}

We thank Jeff Dale, Ed Boyle, and Kenneth Smith of the U.S. Department of the Navy for providing information on wells and culverts, as well as historical information regarding the NAWC site. We also thank Steven Feldmann and Joel Dadepogu of EA Engineering, Science, and Technology, Inc., for providing water-quality data and water-level data. The assistance of Nicholas Smith, Timothy Oden, and Robert Rosman of the U.S. Geological Survey in collecting field data also is appreciated.

\section{DESCRIPTION OF GROUND-WATER-FLOW MODEL}

A three-dimensional, finite-difference ground-water-flow model was used to simulate ground-water flow in and near the NAWC. The model used the MODFLOW (McDonald and Har- baugh, 1988) code for the simulation. A particletracking post-processor--MODPATH (Pollock, 1989)--was used to compute flowpaths for nopumping and recovery-well-pumping scenarios.

Simulations of steady-state and transient ground-water conditions were made. In steadystate conditions, water levels are static and the amount of water stored in the ground-water system remains constant. Steady state is approximated during periods of average precipitation and when other stresses, such as ground-water withdrawals, have remained steady over a long period. Three steady-state simulations were used in this study. One simulation represents a scenario before any recovery-well network was installed and no wells were pumped at the NAWC. The other two steadystate simulations represent scenarios when a recovery-well network has been operating steadily for at least a month. All three simulations include steady pumping at the Roller Bearing Corporation (RBC, fig. 1) of $3,850 \mathrm{ft}^{3} / \mathrm{d}$.

Transient models are appropriate when the amount of water stored in aquifers is changing, as happens when precipitation or pumping rates are changing. Three transient models were used this study for calibration only. Each of these models represented aquifer tests at the NAWC in which single wells were pumped for 3 days or less.

\section{Grid and Boundary Conditions}

The finite-difference model used in this study is discretized into interconnected rectangular cells, each having its own hydrologic characteristics. The cells form a grid comprising 154 rows, 160 columns, and 71 layers. The large number of layers are needed to simulate the effects of the anisotropy caused by the steeply dipping, heterogeneous layers of the ground-water system. Cells within the NAWC property are 25 to $50 \mathrm{ft}$ wide and 25 to $50 \mathrm{ft}$ long. Elsewhere, the cells are larger, with a maximum size of 500 by $650 \mathrm{ft}$ near the model boundaries. The horizontal discretization of the entire model area is shown in figure 4. The horizontal discretization of the model in the vicinity of the NAWC showing locations of wells and streams is shown in figure 5. The model grid is oriented along the approximate strike of the bedding units. 

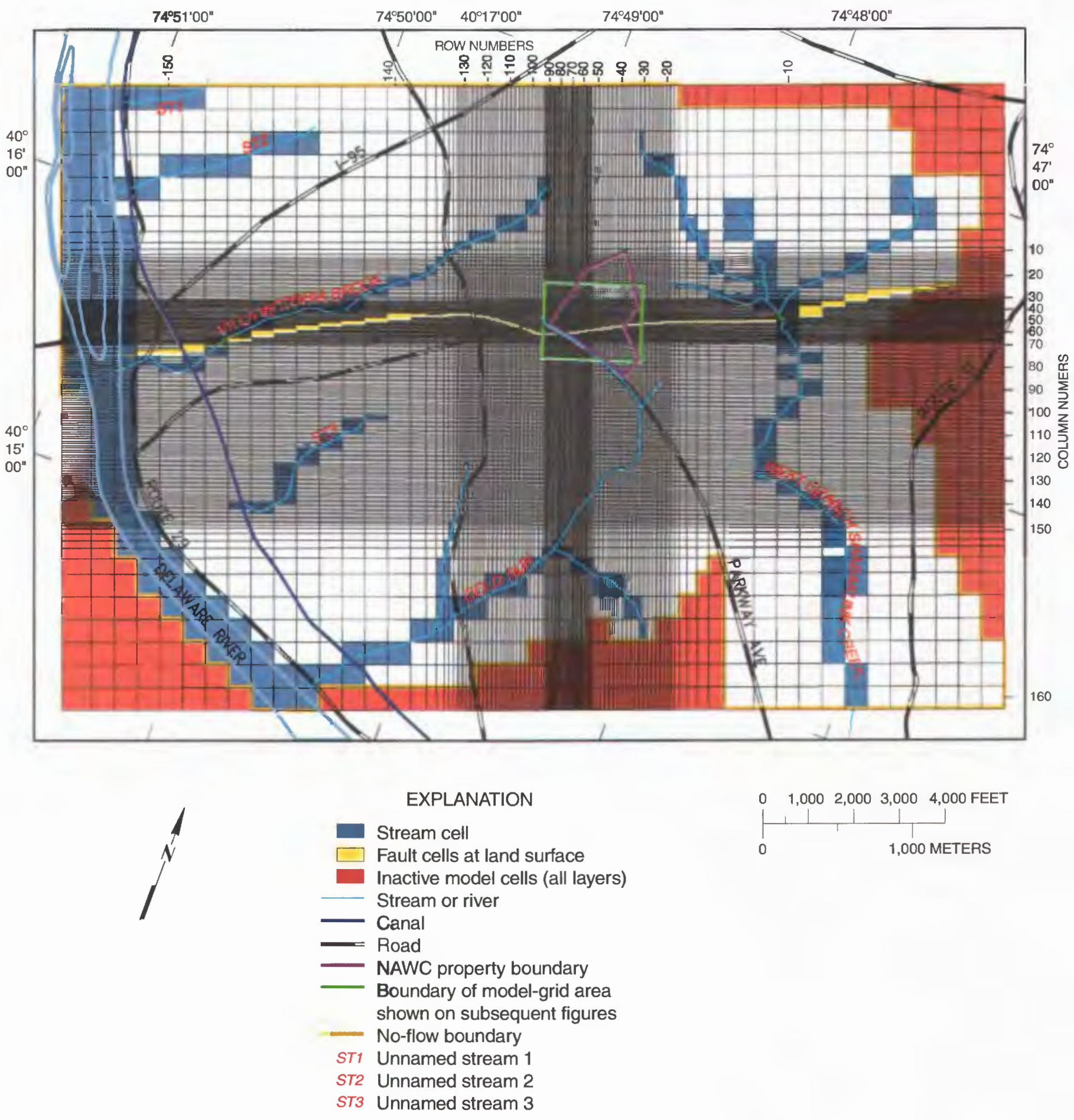

Figure 4. Finite-difference grid for the ground-water-flow model, Naval Air Warfare Center (NAWC), West Trenton, New Jersey, and vicinity. 


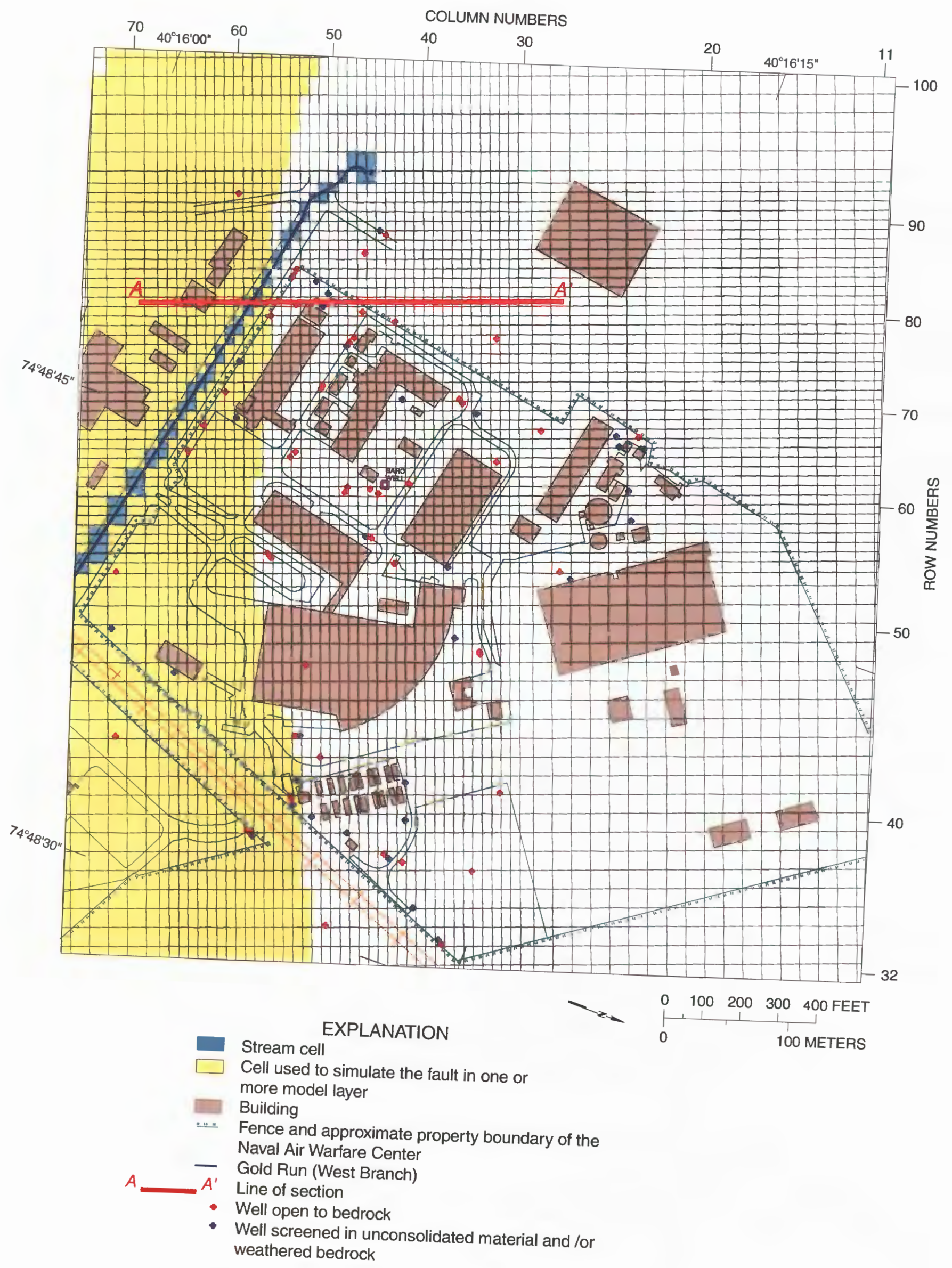

Figure 5. Part of the ground-water-flow model grid showing cells used to simulate Gold Run and the fault in the area of the Naval Air Warfare Center, West Trenton, New Jersey. 
The size of cells in the NAWC area was set at 25 to $50 \mathrm{ft}$ for two reasons. First, cell spacing in the area of interest should be small enough to simulate the shape of cones of depression around pumped wells. During the three short-term pumping periods used in calibrating this model, the smallest cone of depression was about $300 \mathrm{ft}$ wide. Cell spacing of $25 \mathrm{ft}$ is adequate to simulate the changes in head over that 300 -ft interval. The other reason for choosing the 25 - $\mathrm{ft}$ spacing relates to the hydrogeologic framework of the site. When fractured-rock aquifers are simulated as a porous medium, the model simulates the net effect of many fractures rather than the effect of each individual fracture. Instead of simulating the contorted path water actually takes through all the fractures within an individual cell, the model simulates only a straight-line path from the point where water enters a cell to the point where it leaves the cell. Consequently, each model cell must contain enough fractures so that the net cell-to-cell flow is correctly simulated. This model represents rocks in which there are at least 12 fractures across each 25 $\mathrm{ft}$ cell. This large number of fractures per cell helps to insure that the net effect of all of the fractures in a cell is simulated.

The strata described by Lacombe (2000) as bedding units L13 to L23 are represented in the model as layers 19 to 29 ; strata described by Lacombe (2000) as bedding units S11 to S15 are represented in the model as continuations of layers 27 to 31 . To avoid confusion for readers familiar with Lacombe's (2000) bedding-unit numbers, his bedding-unit numbers as well as the model layer numbers representing those units will be used for the remainder of this report. Because the model extends beyond the NAWC area, additional layers were needed updip and downdip of the layers described by Lacombe (2000). These additional model layers are numbers 2 to 18 and 32 to 71 . Thicknesses of these additional layers range from 30 to $60 \mathrm{ft}$, similar to the thicknesses of the bedding units at the NAWC.

Model layer 1 represents the highly weathered zone near land surface. The weathered zone actually is the shallow part of each bedding unit. It is modeled as one continuous layer that is parallel to land surface rather than as part of each bedding unit because its hydrologic properties are different from those of the less weathered material below it. The top of model layer 1 was set at $8.5 \mathrm{ft}$ below land surface on the basis of the mean depth to water in shallow wells. Although the transition between weathered and unweathered rocks is gradational, the bottom of model layer 1 was set at 25 $\mathrm{ft}$ below land surface on the basis of aquifer-test data (discussed in the "Horizontal hydraulic conductivity" section later on). Consequently, model layer 1 was assigned a constant thickness of $16.5 \mathrm{ft}$. Although the weathered zone is unconfined, it is represented in the model as a confined layer. In the model code, the difference between an unconfined and confined layer is that, in unconfined layers, transmissivity varies with the computed thickness of the saturated zone, whereas in confined layers transmissivity is constant. On the basis of measured water levels, the mean thickness of the weathered zone is $16.5 \mathrm{ft}$ and the standard deviation is $3.3 \mathrm{ft}$. This small variation in actual saturated thickness does not appreciably affect computed flowpaths.

The vertical structure of the model is shown in figure 6 . The section shown in this figure is the part of the model that represents the geologic section shown in figure 3 .

Each model layer represents a bedding unit that is composed of numerous water-bearing units and semiconfining units. The impedance to vertical flow caused by the semiconfining units is represented in the model by assigning low vertical hydraulic conductivity to each layer, as described in the later section "Vertical hydraulic conductivity".

Representation of bedding units as model layers is complicated by the dip of the units. Each model layer must be present over the full area of the model even though the bedding units may not be. Each bedding unit terminates updip at the bottom of the weathered zone. Although each bedding unit probably extends thousands of feet downdip, ground-water flow was assumed to be negligible at depths greater than $500 \mathrm{ft}$ below land surface, as explained in the later section "Horizontal hydraulic conductivity." In order to represent these changes in the character of each bedding unit, each model layer is made up of three zones. The most downdip of 


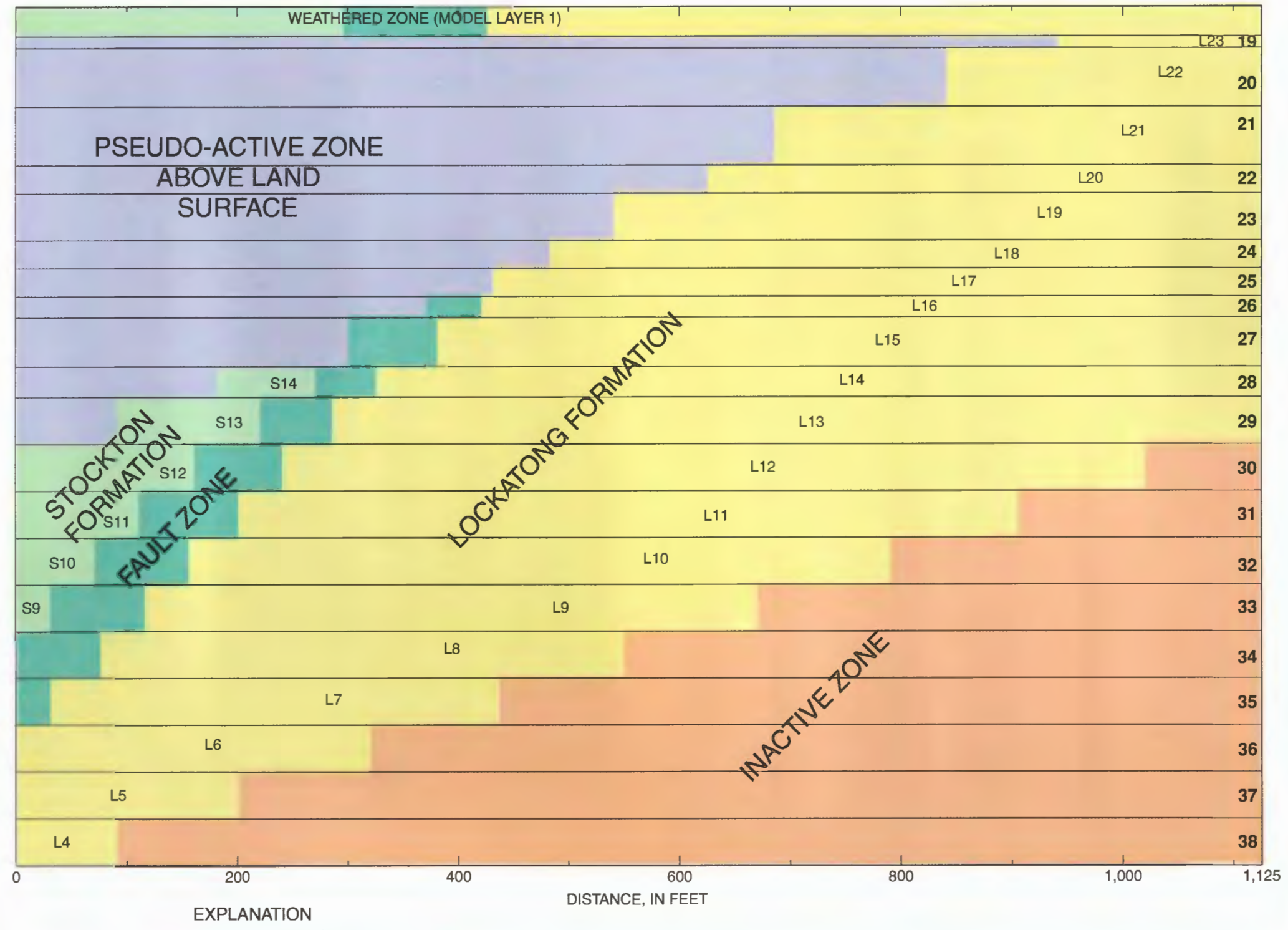

L15 Bedding unit number as 22 Model layer number defined by Lacombe $(2000)$

Figure 6. Section showing vertical discretization of ground-water-flow model along geologic section A-A' at the Naval Air Warfare Center, West Trenton, New Jersey. (Location of section shown on figs. 2 and 5). 
these zones is inactive, representing the part of the bedding unit deeper than $500 \mathrm{ft}$. The next zone is active, representing the part of the bedding unit between the weathered zone and $500 \mathrm{ft}$ below land surface. The next zone is pseudoactive and represents the extension of the bedding unit beyond the outcrop area and above land surface. This zone is composed of pass-through nodes that allow water to flow vertically from the weathered zone (model layer 1) to the bedding unit that actually lies directly under the weathered zone (figs. 3 and 6).

The model extends as far as $1.8 \mathrm{mi}$ beyond the NAWC property. In areas outside of the NAWC, the hydrogeologic framework was assumed to be the same as at the NAWC. No hydrogeologic data outside NAWC were collected by Lacombe; however, Vecchioli and Palmer (1962), in their description of the geology of Mercer County, did not note any variation in the framework.

The model boundaries were chosen to ensure that the entire flowpath of any water that passes through the NAWC property is included in the model. Most of the model boundaries represent natural hydrologic boundaries. The western model boundary is the Delaware River. The north-central, northeastern, eastern, and southern boundaries are defined by the boundaries of the drainage basins of Villa Victoria Brook, West Branch Shabakunk Creek, and Gold Run (fig. 1). The northwestern model boundary is a line extending along strike from the northernmost point of the Villa Victoria Brook drainage basin to the Delaware River. It passes through the drainage basins of the two relatively small streams referred to as unnamed streams 1 and 2 (fig. 1). This line represents the hypothetical flowpath of water that recharges at the northernmost point of the Villa Victoria drainage basin and flows west-southwest to the unnamed streams or the Delaware River.

\section{Hydrologic Parameters}

Hydrologic parameters used in the digital model were based on field measurements made in the model area. Parameters that cannot be measured directly initially were estimated on the basis of values used in digital models of similar areas. During model calibration (discussed later in the
"Calibration" section), all of the parameters were adjusted within reasonable ranges.

\section{Horizontal Hydraulic Conductivity}

Horizontal hydraulic conductivity was measured at 72 well sites at the NAWC between March 15 and July 15, 1997, by means of slug testing. The testing was done by instantaneousl.' placing a solid-body slug into the well to raise the water level and by observing the rate of recovery of the water level. The slug then was instantaneously removed from the well to lower the water level, and the rate of recovery was again observed. The change in water levels was measured with a pressure transducer in the well and recorded with a data logger.

Slug testing is an expeditious method of measuring horizontal hydraulic conductivity in contaminated areas. The minimal disturbance to the flow system in the aquifer minimizes the potential for moving contaminated water into uncontaminated areas. Also, because no water is removed from the well, disposal of contaminated water is unnecessary. Because slug testing displa?es only a small amount of water (less than 1 gallon during the tests at NAWC), however, the area of influence of the test is limited to the area immediately surrounding the well screen or open interval--probably less than a 5-ft radius--and th? value of hydraulic conductivity derived from analyzing the results of a slug test applies only to that small area. Consequently, the purpose of slug testing at the NAWC site was to gain information of the variability of hydraulic conductivity at the site and to determine whether any spatial trends in hydraulic conductivity are present.

The slug tests in the shallow wells (less than $25 \mathrm{ft}$ deep) were analyzed with the method presented by Hvorslev (1951). In this method, it is assumed that the aquifer is unconfined, homogeneous, and infinite in extent. The assumption that the aquifer is unconfined probahly is valid for these shallow wells. The assumptions that the aquifer is homogeneous and infinite probably are valid over the small area influenced by the slug test. 
The analytical method of Cooper and others (1967) was used to calculate horizontal hydraulic conductivity for wells $25 \mathrm{ft}$ deep or deeper. The Cooper method is based on three assumptions: (1) the aquifer is confined and has an infinite areal extent, (2) the aquifer is homogeneous, isotropic, and of uniform thickness over the area influenced by the slug test, and (3) the tested well completely penetrates the aquifer. These assumptions probably are met to an acceptable degree. It is probable that most of the wells greater than $25 \mathrm{ft}$ deep are in parts of the aquifer confined by either the overlying weathered zone or an overlying semiconfining unit. The assumptions that the aquifer has an infinite areal extent and that it is homogeneous, isotropic, and of uniform thickness probably are valid over the small area influenced by each slug test.

The assumption that the well opening fully penetrates the aquifer is probably met for the wells $25 \mathrm{ft}$ deep or deeper. The reason for this requirement in the Cooper method is to minimize the influence of parts of the aquifer that are above or below the well opening. The Lockatong and Stockton Formations consist of many thin waterbearing units alternating with thin semiconfining units. In wells $25 \mathrm{ft}$ deep or deeper, where the rocks are less fractured and less weathered than shallower rocks, is it likely that one of the thin semiconfining units is at or near the top and bottom of each well opening and that the full-penetration assumption is adequately met.

At six wells tested at the NAWC, water levels changed very slowly after the slug was inserted or removed from the well. The water level in these wells changed much more slowly than in the well where the lowest hydraulic conductivity, $0.13 \mathrm{ft} / \mathrm{d}$, was measured. Consequently, the hydraulic conductivity at the six slower-responding wells was probably $0.01 \mathrm{ft} / \mathrm{d}$ or lower; therefore, a value of $0.001 \mathrm{ft} / \mathrm{d}$ for hydraulic conductivity is estimated for these wells.

Two deep wells, well 43BR and well 44BR, were not slug tested. The water level in these wells had not recovered to reasonable static levels during the 2-month period after the wells had been installed and developed. Because of the slow recovery rates in these wells, a hydraulic conductivity of $0.001 \mathrm{ft} / \mathrm{d}$ is estimated for the se wells for purposes of determining trends in hydraulic conductivity over the NAWC area. Six wells at the NAWC site were not slug tested because they were inaccessible, and 12 wells were not tested because they were installed after sling testing was completed. Results of the slug tests and the wells arbitrarily assigned a hydraulic conductivity of $0.001 \mathrm{ft} / \mathrm{d}$ are listed in table 1 .

The slug-test data indicate that hydra'lic conductivity is a function of depth below land surface, as is evident from table 2 . The medien hydraulic conductivity of all wells at depths less than $26 \mathrm{ft}$ is $2.6 \mathrm{ft} / \mathrm{d}$, whereas the median hydraulic conductivity at depths from 26 to $50 \mathrm{ft}$ below land surface is $11.3 \mathrm{ft} / \mathrm{d}$. The lower hydraulic conductivities in the weathered zone probably result from the presence of clay and silt in fractures.

The median hydraulic conductivity $\mathrm{a}^{+}$ depths between 51 and $100 \mathrm{ft}$ below land surface is lower than in the 26- to 50-ft range. Although only six wells are deeper than $100 \mathrm{ft}$, the data from those wells indicate that hydraulic conductivity continues to decrease at depths greater than $100 \mathrm{ft}$. A previous study (Lewis-Brown and Jacobsen, 1995) of specific-capacity data from wells throughout the southern part of the Newark Basin in New Je"sey reported similar results. In the 348 wells open to the Lockatong Formation, specific capacity per foot of open hole decreases by two orders of magnitude (from 0.012 to $0.00011[(\mathrm{gal} / \mathrm{min}) / \mathrm{ft}] / \mathrm{ft}$ ) as well depth increases from $75 \mathrm{ft}$ to greater than $300 \mathrm{ft}$. In 271 wells open to the Stockton Formation, specific capacity per foot of open hole decreases by ahout one order of magnitude (from 0.032 to 0.00393 $[(\mathrm{gal} / \mathrm{min}) / \mathrm{ft}] / \mathrm{ft})$ as well depth increases from $75 \mathrm{ft}$ to over $300 \mathrm{ft}$. This decrease in hydraulic conductivity may indicate the presence of fever fractures and fewer interconnected fractures with depth. Knopman (1990) suggests the alternat've explanation that deep wells may be complete $\mathrm{f}$ in areas where the yield at shallow depths is poor, and holes were drilled deeper in search of better yields. Although Knopman's explanation may be applicable to water-supply wells, it does not explain the decrease in hydraulic conductivity found in observation wells at the NAWC. Therefore, 
Table 1. Horizontal hydraulic conductivity at, and well construction data for, wells at and near the Naval Air Warfare Center, West Trenton, New Jersey

Latitude and longitude are referenced to the North American Datum of 1927; nm, hydraulic conductivity was not measured or estimated at this well site; est .001 , hydraulic conductivity estimated to be 0.001 feet per day at this well site; na, not applicable; --, data not available; WZ, weathered zone;

L, Lockatong Formation; S, Stockton Formation; USGS, U.S. Geological Survey]

\begin{tabular}{|c|c|c|c|c|c|c|c|c|c|c|c|}
\hline \multirow{2}{*}{$\begin{array}{c}\text { Well } \\
\text { name }\end{array}$} & \multirow{2}{*}{$\begin{array}{c}\text { USGS } \\
\text { well } \\
\text { num- } \\
\text { ber }\end{array}$} & \multirow{2}{*}{$\begin{array}{l}\text { New } \\
\text { Jersey } \\
\text { permit } \\
\text { number }\end{array}$} & \multirow{2}{*}{ Latitude } & \multirow{2}{*}{$\begin{array}{l}\text { Longi- } \\
\text { tude }\end{array}$} & \multirow{2}{*}{$\begin{array}{c}\text { Alti- } \\
\text { tude of } \\
\text { land } \\
\text { sur- } \\
\text { face } \\
\text { (feet } \\
\text { above } \\
\text { sea } \\
\text { level) }\end{array}$} & \multicolumn{2}{|c|}{$\begin{array}{c}\text { Depth of open } \\
\text { interval (feet } \\
\text { below land } \\
\text { surface) } \\
\end{array}$} & \multirow{3}{*}{$\begin{array}{c}\text { Primary } \\
\text { bedding } \\
\text { unit } \\
\text { (Lacombe, } \\
2000 \text { ) }\end{array}$} & \multirow{3}{*}{$\begin{array}{l}\text { Model } \\
\text { layer } \\
\text { number }\end{array}$} & \multirow{3}{*}{$\begin{array}{l}\text { For- } \\
\text { ma- } \\
\text { tion }\end{array}$} & \multirow{2}{*}{$\begin{array}{l}\text { Horizontal } \\
\text { hydraulic } \\
\text { cor ductivity } \\
\text { (feet per } \\
\text { day) }\end{array}$} \\
\hline & & & & & & Top & $\begin{array}{l}\text { Bot- } \\
\text { tom }\end{array}$ & & & & \\
\hline \multicolumn{9}{|c|}{ WELLS AT THE NAVAL AIR WARFARE CENTER } & & & \\
\hline $1 \mathrm{~S}$ & 210492 & $27-09892-3$ & 401611 & 744849 & 150.87 & 3 & 13 & WZ & 1 & $\mathbf{L}$ & 0.2 \\
\hline $2 S$ & 210493 & $27-09895-8$ & 401607 & 744849 & 149.82 & 3 & 8 & WZ & 1 & $\mathbf{L}$ & 3.5 \\
\hline $4 S$ & 210494 & $27-09894-0$ & 401605 & 744851 & 150.21 & 3 & 7 & WZ & 1 & $\mathbf{L}$ & $\mathrm{nm}$ \\
\hline $6 S$ & 210495 & 27-09893-1 & 401604 & 744847 & 147.88 & 4 & 10 & WZ & 1 & $\mathbf{S}$ & 10.2 \\
\hline $11 \mathrm{~S}$ & 210496 & $27-09888-5$ & 401612 & 744835 & 159.82 & 8 & 23 & WZ & 1 & $L$ & 1.7 \\
\hline $12 S$ & 210497 & $27-09889-3$ & 401612 & 744833 & 158.82 & 10.5 & 20.5 & WZ & 1 & $L$ & 3.6 \\
\hline $13 S$ & 210498 & $27-09890-7$ & 401613 & 744832 & 159.35 & 10 & 20 & WZ & 1 & $L$ & 35.6 \\
\hline $14 S$ & 210499 & $27-09891-5$ & 401614 & 744831 & 163.89 & 14.5 & 24.5 & WZ & 1 & $\mathbf{L}$ & 2.6 \\
\hline $15 S$ & 210500 & $27-09918-1$ & 401608 & 744848 & 149.72 & 3 & 13 & WZ & 1 & $L$ & 3.7 \\
\hline $16 S$ & 210501 & 27-09919-9 & 401611 & 744844 & 150.25 & 2 & 12 & WZ & 1 & $L$ & 5.9 \\
\hline $17 \mathrm{~S}$ & 210502 & $27-09920-2$ & 401609 & 744843 & 149.99 & 3 & 8 & WZ & 1 & $L$ & 1.6 \\
\hline $18 \mathrm{~S}$ & 210503 & 27-09921-1 & 401616 & 744848 & 169.74 & 6 & 16 & WZ & 1 & $L$ & 8.1 \\
\hline $19 S$ & 210504 & $27-09922-9$ & 401615 & 744847 & 169.88 & 7 & 17 & WZ & 1 & $L$ & 11.1 \\
\hline $20 S$ & 210505 & $27-09923-7$ & 401615 & 744848 & 169.13 & 4 & 19 & WZ & 1 & $L$ & est .001 \\
\hline $24 S$ & 210507 & $27-09927-0$ & 401615 & 744850 & 169.38 & 5.5 & 15.5 & WZ & 1 & $L$ & 2.5 \\
\hline $25 S$ & 210508 & $27-09925-3$ & 401614 & 744849 & 168.80 & 3.5 & 18.5 & WZ & 1 & $L$ & .21 \\
\hline $26 S$ & 210509 & 27-09926-1 & 401614 & 744850 & 169.13 & 6.3 & 16.3 & WZ & 1 & $L$ & 1.4 \\
\hline $27 \mathrm{~S}$ & 210510 & $27-10960-7$ & 401612 & 744836 & 162.26 & 11.2 & 21.2 & WZ & 1 & L & 1.5 \\
\hline $28 \mathrm{~S}$ & 210511 & $27-10962$ & 401611 & 744834 & 157.30 & 10 & 25 & WZ & 1 & $L$ & 11.8 \\
\hline $29 \mathrm{~S}$ & 210512 & $27-10982-8$ & 401609 & 744834 & 156.95 & 10 & 20 & WZ & 1 & $\mathbf{S}$ & 13.9 \\
\hline $30 S$ & 210513 & $27-10964$ & 401609 & 744835 & 158.68 & 7.5 & 17.5 & WZ & 1 & $S$ & 34.3 \\
\hline $31 S$ & 210601 & $27-10963$ & 401609.06 & 744836.38 & 150.72 & 10 & 20 & WZ & 1 & $L$ & 1.6 \\
\hline $32 S$ & 210418 & $27-12423$ & 401606 & 744853 & 153.55 & 5 & 15 & WZ & 1 & $L$ & 3.8 \\
\hline $33 S$ & 210419 & $27-12424$ & 401600 & 744846 & 155.15 & 6 & 16 & WZ & 1 & $\mathbf{S}$ & .3 \\
\hline $34 S$ & 210420 & $27-12425$ & 401603 & 744838 & 144.82 & 8 & 18 & WZ & 1 & $S$ & .13 \\
\hline $35 S$ & 210421 & $27-12426$ & 401609 & 744833 & 156.15 & 5 & 15 & WZ & 1 & $\mathrm{~s}$ & 7.9 \\
\hline $36 S$ & 210549 & $27-12680$ & 401613 & 744844 & 170.61 & 3 & 13 & WZ & 1 & $\mathbf{S}$ & $\mathrm{nm}$ \\
\hline $37 \mathrm{~S}$ & 210528 & $27-12681$ & 401605 & 744837 & 148.34 & 6 & 16 & WZ & 1 & $\mathbf{S}$ & .22 \\
\hline $38 \mathrm{~S}$ & 210600 & $27-12682$ & 401610 & 744834 & 150 & 2 & 7 & WZ & 1 & $L$ & 1.8 \\
\hline $39 \mathrm{~S}$ & 210529 & $27-12683$ & 401605 & 744846 & 150.05 & 3 & 13 & WZ & 1 & $\mathrm{~S}$ & .6 \\
\hline
\end{tabular}


Table 1. Horizontal hydraulic conductivity at, and well construction data for, wells at and near the Naval Air Warfare Center, West Trenton, New Jersey--Continued

Latitude and longitude are referenced to the North American Datum of 1927; nm, hydraulic conductivity was not measured or estimated at this we" site; est .001, hydraulic conductivity estimated to be 0.001 feet per day at this well site; na, not applicable; --, data not available; WZ, weathered zone;

L, Lockatong Formation; S, Stockton Formation; USGS, U.S. Geological Survey]

\begin{tabular}{|c|c|c|c|c|c|c|c|c|c|c|c|}
\hline \multirow{2}{*}{$\begin{array}{c}\text { Well } \\
\text { name }\end{array}$} & \multirow{2}{*}{$\begin{array}{c}\text { USGS } \\
\text { well } \\
\text { num- } \\
\text { ber }\end{array}$} & \multirow{2}{*}{$\begin{array}{c}\text { New } \\
\text { Jersey } \\
\text { permit } \\
\text { number }\end{array}$} & \multirow{2}{*}{ Latitude } & \multirow{2}{*}{$\begin{array}{l}\text { Longi- } \\
\text { tude }\end{array}$} & \multirow{2}{*}{$\begin{array}{c}\text { Alti- } \\
\text { tude of } \\
\text { land } \\
\text { sur- } \\
\text { face } \\
\text { (feet } \\
\text { above } \\
\text { sea } \\
\text { level) }\end{array}$} & \multicolumn{2}{|c|}{$\begin{array}{c}\text { Depth of open } \\
\text { interval (feet } \\
\text { below land } \\
\text { surface) }\end{array}$} & \multirow{2}{*}{$\begin{array}{c}\text { Primary } \\
\text { bedding } \\
\text { unit } \\
\text { (Lacombe, } \\
\text { 2000) }\end{array}$} & \multirow{2}{*}{$\begin{array}{c}\text { Model } \\
\text { layer } \\
\text { number }\end{array}$} & \multirow{2}{*}{$\begin{array}{l}\text { For- } \\
\text { ma- } \\
\text { tion }\end{array}$} & \multirow{2}{*}{$\begin{array}{l}\text { Horizontal } \\
\text { hydraulic } \\
\text { c-nductivity } \\
\text { (feet per } \\
\text { day) }\end{array}$} \\
\hline & & & & & & Top & $\begin{array}{l}\text { Bot- } \\
\text { tom }\end{array}$ & & & & \\
\hline
\end{tabular}

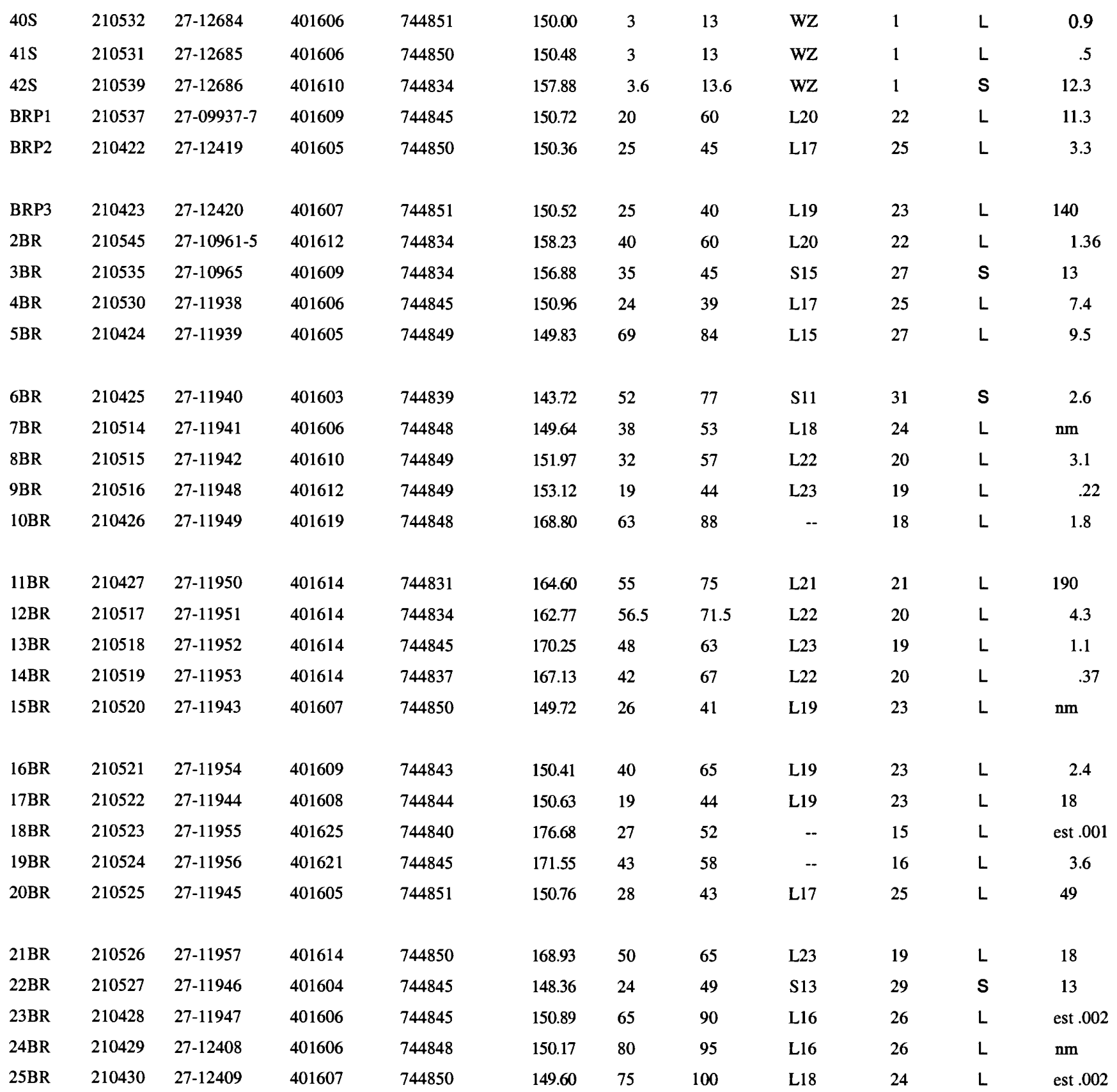


Table 1. Horizontal hydraulic conductivity at, and well construction data for, wells at and near the Naval Air Warfare Center, West Trenton, New Jersey--Continued

Latitude and longitude are referenced to the North American Datum of 1927; nm, hydraulic conductivity was not measured or estimated at this well site; est .001, hydraulic conductivity estimated to be 0.001 feet per day at this well site; na, not applicable; --, data not available; WZ, weathered zone;

$\underline{\text { L, Lockatong Formation; S, Stockton Formation; USGS, U.S. Geological Survey] }}$

\begin{tabular}{|c|c|c|c|c|c|c|c|c|c|c|c|}
\hline \multirow{2}{*}{$\begin{array}{c}\text { Well } \\
\text { name }\end{array}$} & \multirow{2}{*}{$\begin{array}{l}\text { USGS } \\
\text { well } \\
\text { num- } \\
\text { ber }\end{array}$} & \multirow{2}{*}{$\begin{array}{c}\text { New } \\
\text { Jersey } \\
\text { permit } \\
\text { number }\end{array}$} & \multirow{2}{*}{ Latitude } & \multirow{2}{*}{$\begin{array}{l}\text { Longi- } \\
\text { tude }\end{array}$} & \multirow{2}{*}{$\begin{array}{l}\text { Alti- } \\
\text { tude of } \\
\text { land } \\
\text { sur- } \\
\text { face } \\
\text { (feet } \\
\text { above } \\
\text { sea } \\
\text { level) }\end{array}$} & \multicolumn{2}{|c|}{$\begin{array}{c}\text { Depth of open } \\
\text { interval (feet } \\
\text { below land } \\
\text { surface) }\end{array}$} & \multirow{2}{*}{$\begin{array}{c}\text { Primary } \\
\text { bedding } \\
\text { unit } \\
\text { (Lacombe, } \\
2000)\end{array}$} & \multirow{2}{*}{$\begin{array}{l}\text { Model } \\
\text { layer } \\
\text { number }\end{array}$} & \multirow{2}{*}{$\begin{array}{l}\text { For- } \\
\text { ma- } \\
\text { tion }\end{array}$} & \multirow{2}{*}{$\begin{array}{l}\text { Horizontal } \\
\text { r'draulic } \\
\text { conductivity } \\
\text { (feet per } \\
\text { day) }\end{array}$} \\
\hline & & & & & & Top & $\begin{array}{l}\text { Bot- } \\
\text { tom }\end{array}$ & & & & \\
\hline
\end{tabular}

\begin{tabular}{|c|c|c|c|c|c|c|c|c|c|c|c|}
\hline $26 \mathrm{BR}$ & 210431 & $27-12410$ & 401605 & 744851 & 150.7 & 80 & 95 & L15 & 27 & $L$ & .27 \\
\hline $27 \mathrm{BR}$ & 210432 & $27-12412$ & 401604 & 744847 & 147.90 & 65 & 80 & $\mathrm{~L} 13$ & 29 & $L$ & est 0.001 \\
\hline $28 \mathrm{BR}$ & 210433 & $27-12413$ & 401604 & 744844 & 148.20 & 76 & 91 & $\mathrm{~S} 12$ & 30 & $S$ & .13 \\
\hline 30BR & 210434 & $27-12428$ & 401608 & 744845 & 150.40 & 85 & 110 & L18 & 24 & $L$ & .22 \\
\hline $32 \mathrm{BR}$ & 210436 & $27-12430$ & 401609 & 744833 & 156.60 & 40 & 55 & $\mathrm{~S} 14$ & 28 & $\mathrm{~S}$ & 5.4 \\
\hline 33BR & 210437 & $27-12414$ & 401607 & 744853 & 153.72 & 30 & 45 & L21 & 21 & $\mathbf{L}$ & 227 \\
\hline 34BR & 210438 & $27-12415$ & 401603 & 744853 & 151.22 & 35 & 48 & L15 & 27 & $L$ & 34.0 \\
\hline $35 \mathrm{BR}$ & 210439 & $27-12416$ & 401600 & 744846 & 155.50 & 31 & 47 & $S 10$ & 32 & $S$ & .45 \\
\hline $38 \mathrm{BR}$ & 210442 & $27-12411$ & 401609 & 744846 & 150.60 & 100 & 115 & L19 & 23 & $L$ & est .001 \\
\hline 39BR & 210534 & $27-13976$ & 401607 & 744842 & 150.05 & 68 & 88 & L15 & 27 & $L$ & 23 \\
\hline 40BR & 210533 & $27-13977$ & 401606 & 744853 & 154.04 & 95 & 120 & L18 & 24 & $L$ & .84 \\
\hline 41BR & 210541 & $27-13978$ & 401610 & 744843 & 150.29 & 85 & 110 & L19 & 23 & $L$ & 47 \\
\hline 42BR & 210551 & $27-13979$ & 401607 & 744842 & 159.81 & 120 & 140 & L13 & 29 & $L$ & 1.3 \\
\hline 43BR & 210546 & $27-13980$ & 401613 & 744841 & 169.02 & 385 & 410 & L13 & 29 & $L$ & est .001 \\
\hline 44BR & 210547 & $27-13981$ & 401613 & 744841 & 168.38 & 305 & 330 & L15 & 27 & $L$ & est .001 \\
\hline $45 \mathrm{BR}$ & 210542 & $27-13982$ & 401610 & 744851 & 159.50 & 185 & 210 & L19 & 23 & $\mathrm{~L}$ & 12.6 \\
\hline $51 \mathrm{BR}$ & 210548 & $27-14150$ & 401613 & 744833 & 159.47 & 86 & 96 & $\mathrm{~L} 20$ & 22 & $L$ & 140 \\
\hline $52 \mathrm{BR}$ & 210576 & $27-15277$ & 401604.02 & 744845.07 & 148.30 & 155 & 180 & -- & 32 & $\mathrm{~L}$ & $\mathrm{~nm}$ \\
\hline 53BR & 210581 & $27-15279$ & 401609.92 & 744840.96 & 148.42 & 95 & 120 & L19 & 23 & $L$ & $\mathrm{~nm}$ \\
\hline 54BR & 210575 & $27-15278$ & 401607.74 & 744835.87 & 153.94 & 175 & 200 & -- & 30 & $\mathrm{~L}$ & $\mathrm{~nm}$ \\
\hline $55 \mathrm{BR}$ & 210582 & $27-15275$ & 401613.10 & 744850.09 & 164.02 & 135 & 160 & L21 & 21 & $L$ & $\mathrm{~nm}$ \\
\hline
\end{tabular}


Table 1. Horizontal hydraulic conductivity at, and well construction data for, wells at and near the Naval Air Warfare Center, West Trenton, New Jersey--Continued

Latitude and longitude are referenced to the North American Datum of $1927 ; \mathrm{nm}$, hydraulic conductivity was not measured or estimated at this woll site; est .001, hydraulic conductivity estimated to be 0.001 feet per day at this well site; na, not applicable; --, data not available; WZ, weathered zone;

L, Lockatong Formation; S, Stockton Formation; USGS, U.S. Geological Survey]

\begin{tabular}{|c|c|c|c|c|c|c|c|c|c|c|c|}
\hline Well & $\begin{array}{c}\text { USGS } \\
\text { well }\end{array}$ & $\begin{array}{l}\text { New } \\
\text { Jersey }\end{array}$ & & & $\begin{array}{l}\text { Alti- } \\
\text { tude of } \\
\text { land } \\
\text { sur- }\end{array}$ & $\begin{array}{r}\text { Depth } \\
\text { interv } \\
\text { belo } \\
\text { sur } \\
\end{array}$ & $\begin{array}{l}\text { open } \\
\text { (feet } \\
\text { land } \\
\text { ce) } \\
\end{array}$ & $\begin{array}{l}\text { Primary } \\
\text { bedding }\end{array}$ & & For- & $\begin{array}{l}\text { Horizontal } \\
\text { hydraulic }\end{array}$ \\
\hline $56 \mathrm{BR}$ & 210579 & $27-15276$ & 401607.66 & 744849.93 & 150.36 & 140 & 165 & L17 & 25 & $L$ & $\mathrm{~nm}$ \\
\hline $57 \mathrm{BR}$ & 210577 & $27-15269$ & 401605.98 & 744854.68 & 151.42 & 12 & 27 & WZ & 1 & $L$ & $\mathrm{~nm}$ \\
\hline $58 \mathrm{BR}$ & 210578 & $27-15270$ & 401606.24 & 744857.81 & 154.89 & 85 & 110 & L18 & 24 & $L$ & $\mathrm{~nm}$ \\
\hline $59 \mathrm{BR}$ & 210573 & - & 401605.74 & 744855.84 & 152.54 & 56 & 80 & L19 & 23 & $L$ & $\mathrm{~nm}$ \\
\hline 11MW1 & 210570 & $27-14458$ & 401606.89 & 744835.78 & 153.15 & 8 & 22 & WZ & 1 & $S$ & $\mathrm{~nm}$ \\
\hline 12MW1 & 210580 & $27-15414$ & 401608.60 & 744834.65 & 156.52 & 5 & 15 & wZ & 1 & $S$ & $\mathrm{~nm}$ \\
\hline $35 \mathrm{MWl}$ & 210572 & $27-14459$ & 401615.89 & 744848.97 & 169.91 & 7 & 25 & WZ & 1 & $L$ & $\mathrm{~nm}$ \\
\hline $35 \mathrm{MW} 2$ & 210571 & $27-14460$ & 401615.48 & 744849.50 & 157.31 & 6.5 & 22.5 & WZ & 1 & $L$ & $\mathrm{~nm}$ \\
\hline \multicolumn{12}{|c|}{ WELLS OUTSIDE NAVAL AIR WARFARE CENTER PROPERTY } \\
\hline MW101 & 210470 & $27-13573$ & 401658 & 744843 & 201.80 & 2.17 & 22.2 & -- & 1 & $L$ & $\mathrm{~nm}$ \\
\hline MW3 & 210586 & $27-09331-0$ & 401458 & 744925 & 91.0 & 44 & 80 & -- & 54 & S & $\mathrm{nm}$ \\
\hline MW4 & 210583 & 24-09332-8 & 401448 & 744929 & 51.5 & 9 & 19 & -- & 1 & $S$ & $\mathrm{~nm}$ \\
\hline MJM5 & 210584 & -- & 401455 & 744855 & 73.42 & -- & 24.8 & -- & 1 & $s$ & $\mathrm{~nm}$ \\
\hline NJM6 & 210587 & -- & 401501 & 744905 & 96.59 & -- & 29.7 & -- & 1 & $s$ & $\mathrm{~nm}$ \\
\hline NJM7 & 210588 & - & 401504 & 744902 & 95.78 & -- & 30 & -- & 1 & $S$ & $\mathrm{~nm}$ \\
\hline NJM11 & 210585 & -- & 401457 & 744903 & 90.16 & -- & 25.4 & -- & 1 & $S$ & $\mathrm{~nm}$ \\
\hline Civil Defense & 210028 & $27-04214$ & 401553 & 745012 & 122.99 & 33.0 & 300.0 & -- & $18-23$ & $L$ & $\mathrm{~nm}$ \\
\hline MW7-17 & 210595 & $27-11594$ & 401532 & 744851 & 126.8 & 7 & 17 & -- & 1 & $S$ & $\mathrm{~nm}$ \\
\hline MW7-80 & 210594 & $27-14055$ & 401532 & 744851 & 126.5 & 60 & 80 & -- & 45 & $S$ & $\mathrm{~nm}$ \\
\hline MW7-250 & 210593 & $27-11484$ & 401532 & 744851 & 127.0 & 240 & 250 & -- & 49 & $S$ & $\mathrm{~nm}$ \\
\hline MW32-25 & 210592 & $27-11612$ & 401526 & 744841 & 109.9 & 13 & 25 & -- & 1 & $S$ & $\mathrm{~nm}$ \\
\hline MW32-57 & 210591 & $27-11659$ & 401526 & 744841 & 110.0 & 37 & 57 & -- & 48 & $S$ & $\mathrm{~nm}$ \\
\hline MW35-25 & 210589 & $27-11614$ & 401523 & 744856 & 118.8 & 8 & 25 & - & 1 & $S$ & $\mathrm{~nm}$ \\
\hline MW35-57 & 210590 & -- & 401523 & 744856 & 118.7 & 37 & 57 & -- & 48 & $S$ & $\mathrm{~nm}$ \\
\hline MW44-30 & 210598 & -- & 401540 & 744835 & 118.2 & 10 & 30 & -- & 1 & s & $\mathrm{nm}$ \\
\hline MW44-80 & 210599 & -- & 401540 & 744835 & 118.2 & 65 & 80 & -- & 44 & $\mathbf{S}$ & $\mathrm{nm}$ \\
\hline MW48-25 & 210596 & -- & 401534 & 744834 & 112.5 & 10 & 25 & -- & 1 & $\mathbf{s}$ & $\mathrm{nm}$ \\
\hline MW48-63 & 210597 & $27-11705$ & 401534 & 744834 & 112.5 & 38.0 & 63.0 & -- & 46 & $\mathbf{s}$ & $\mathrm{nm}$ \\
\hline
\end{tabular}


Table 2. Horizontal hydraulic conductivity as a function of depth below land surface, Naval Air Warfare Center, West Trenton, New Jersey

[na, not applicable]

\begin{tabular}{|c|c|c|c|c|c|c|}
\hline \multirow{2}{*}{$\begin{array}{l}\text { Depth of cen- } \\
\text { ter of screen or } \\
\text { open interval } \\
\text { (feet below land } \\
\text { surface) }\end{array}$} & \multirow[t]{2}{*}{$\begin{array}{l}\text { Number } \\
\text { of wells }\end{array}$} & \multicolumn{2}{|c|}{$\begin{array}{c}\text { Horizontal hydraulic } \\
\text { conductivity } \\
\text { (feet per day) }\end{array}$} & \multirow{2}{*}{\multicolumn{3}{|c|}{$\begin{array}{l}\text { Horizontal hydraulic conductivity used in model } \\
\text { (feet per day) }\end{array}$}} \\
\hline & & Range & Median & & & \\
\hline \multicolumn{7}{|c|}{ ALL WELLS } \\
\hline $\begin{array}{l}0-25 \text { (weath- } \\
\text { ered zone) }\end{array}$ & 32 & $0.001-35.6$ & 2.6 & & na & \\
\hline $26-50$ & 17 & $.001-227$ & 11.3 & & na & \\
\hline $51-100$ & 24 & $.001-190$ & 3.1 & & na & \\
\hline $101-300$ & 5 & $.001-126$ & .40 & & na & \\
\hline $301-500$ & 2 & $.001-.001$ & .001 & & na & \\
\hline \multicolumn{7}{|c|}{ LOCKATONG FORMATION } \\
\hline $\begin{array}{l}0-25 \text { (weath- } \\
\text { ered zone) }\end{array}$ & 23 & $.001-35.6$ & 2.5 & & 2.5 & \\
\hline $26-50$ & 13 & $.001-227$ & 11.3 & & 11.3 & \\
\hline $51-100$ & 21 & $.001-190$ & 4.3 & & 4.0 & \\
\hline $101-300$ & 5 & $.001-12.6$ & .84 & & .4 & \\
\hline $301-500$ & 2 & $.001-.001$ & .001 & & .001 & \\
\hline All Lockatong & 64 & $.001-227$ & 3.2 & & & \\
\hline \multicolumn{7}{|c|}{ FAULT ZONE } \\
\hline All fault cells & 0 & na & na & & .001 & \\
\hline \multicolumn{7}{|c|}{ STOCKTON FORMATION } \\
\hline & & & & $\begin{array}{l}\text { North } \\
\text { (columns 28-79) }\end{array}$ & $\begin{array}{l}\text { Central } \\
\text { (columns 80-110) }\end{array}$ & $\begin{array}{c}\text { Southern } \\
\text { (columns 111- } \\
160)\end{array}$ \\
\hline $\begin{array}{l}0-25 \text { (weathered } \\
\text { zone) }\end{array}$ & 9 & $.13-34.3$ & 7.9 & 1.25 & 1.75 & 5.0 \\
\hline $26-50$ & 4 & $.45-13$ & 9.2 & 1.13 & 1.75 & 5.0 \\
\hline $51-100$ & 3 & $.13-2.6$ & .32 & .375 & .625 & 1.75 \\
\hline $101-300$ & 0 & -- & -- & .0375 & .0625 & .175 \\
\hline $301-500$ & 0 & -- & -- & .001 & .001 & .001 \\
\hline All Stockton & 16 & $.13-34.3$ & 4.0 & & & \\
\hline
\end{tabular}


for purposes of the ground-water-flow model used in this study, it was assumed that hydraulic conductivity does decrease with depth and that, at depths below $500 \mathrm{ft}$, hydraulic conductivity virtually is zero.

The hydraulic conductivity at depths less than $26 \mathrm{ft}$ is more uniform than in deeper wells. This greater uniformity in the weathered zone probably is a result of more homogeneous conditions because fractures are filled with silt and clay. The higher variability below the weathered zone, where fractures are open, is not surprising, given the nature of the fractured-rock aquifer. Wells in which high hydraulic conductivities (greater than $25 \mathrm{ft} / \mathrm{d}$ ) were measured probably are completed where one or more vertical fractures intersect bedding-plane fractures, whereas wells with low hydraulic conductivities (less than $0.01 \mathrm{ft} / \mathrm{d}$ ) probably are between vertical fractures. This high degree of variability is not represented in the model. Rather, the rock units were modeled as porous media, with fractures being the pores and each cell containing many fractures. The hydraulic conductivity of a given cell is a function of the net effect of all interconnected fractures in the cell.

On the basis of the hydraulic conductivity data, the bottom of the weathered zone was set in the model at $25 \mathrm{ft}$ below land surface. In the model, hydraulic conductivity was initially set at the median values obtained from slug testing. On the basis of calibration, the hydraulic conductivities were adjusted slightly to achieve the optimum match between measured and simulated groundwater levels and base flow in streams. The final values used in simulations and flow-path analyses are listed in table 2. A complete description of the model-calibration process is given later in the "Calibration" section.

Hydraulic conductivity data are sparse for the model area beyond the NAWC. Various regional investigations have reported that, in general, the Stockton Formation is more transmissive than the Lockatong Formation (Vecchioli and Palmer, 1962; Kasabach, 1966; Houghton, 1990). Data from an aquifer test at the General Motors Corporation (now Delphi, fig. 1) indicate that the hydraulic conductivity of the weathered zone in that area ranges from 8.0 to $31.0 \mathrm{ft} / \mathrm{d}$ (Roy F. Weston, Inc., 1989). Data from an aquifer test at the Roller Bearing Corporation (RBC, fig. 1) indicate that the median hydraulic conductivity in that area is about $41.0 \mathrm{ft} / \mathrm{d}$ in the weathered zone and about $20 \mathrm{f} / / \mathrm{d}$ in the 60- to 80-ft-depth range (Rare Earth Envirosciences, Inc., 1992). During each of these aquifer tests, less than $10 \mathrm{gal} / \mathrm{min}$ of water was pumped, so only a small area around the scree $\eta$ of the pumped well was influenced by the pumping. Therefore, the hydraulic conductivity at each of the aquifer-test sites could be estimated by dividing the reported transmissivity by the length of the sceen of the pumped well.

The data for the Stockton Formation indicate that the hydraulic conductivity of the formation is higher in the southern part of the study area than it is in the northern part of the area, near the contact with the Lockatong Formation. The contact between the Stockton and Lockatong Formations is gradational, so a gradual change in hydraulic properties with distance from the contact is not surprising.

The horizontal hydraulic conductivity of the fault zone was assumed to be extremely low on the basis of evidence that only a small amount of ground water moves across the fault, as descrited in the earlier section "Hydrogeologic framework." During model calibration, the best match betv'een measured and simulated heads and base flow was achieved when the horizontal hydraulic conductivity of the fault zone was set to $0.001 \mathrm{ft} / \mathrm{d}$.

During model calibration, the best match between measured and simulated heads and b?se flow was achieved when the Stockton formation was divided into three zones. The northern zone includes the NAWC area and comprises mode ${ }^{1}$ columns 28-79, the central zone includes the General Motors area and comprises model colımns 80 to 110 , and the southern zone includes the R oller Bearing Corporation area and comprises model columns 111 to 160 . The horizontal hydraulic conductivities used in the final calibrated mocel in the Stockton Formation are listed in table 2.

Cells in the pseudoactive zone described in the earlier section "Grid and boundary conditions" 
were assigned a horizontal hydraulic conductivity of zero. Only vertical movement of water is represented in this zone because the purpose of this zone is to allow water to move directly from the weathered zone down to whatever model layer represents the bedding unit directly below the weathered zone.

\section{Vertical Hydraulic Conductivity}

Vertical hydraulic conductivity cannot be readily measured in the field, especially in fractured rock. Vertical hydraulic conductivity is assumed to be lower than horizontal hydraulic conductivity because horizontal fractures are more numerous than vertical fractures per unit volume of aquifer, and the horizontal fractures are more continuous. The vertical hydraulic conductivity of each model cell initially was set at one-tenth the horizontal hydraulic conductivity of the cell. Although the fraction was adjusted during model calibration, the best match between measured and simulated ground-water levels and stream discharge was achieved when the vertical hydraulic conductivity was set at one-tenth the horizontal hydraulic conductivity. Vertical conductance, the parameter actually used in the model, was calculated in a computer program (not part of the model) by dividing the vertical hydraulic conductivity at each cell by the thickness of the bedrock unit represented by the cell.

Cells in the pseudoactive zone were assigned a vertical conductance of $100 \mathrm{ft} / \mathrm{d}$ to insure instantaneous movement of water through the pseudoactive zone from the weathered zone to the model layer that represents the bedding unit directly beneath the weathered zone.

The rate of flow between a stream and the aquifer is affected primarily by the vertical hydraulic conductivity and thickness of the streambed material. Because the vertical hydraulic conductivity of a streambed is difficult to measure in the field, this parameter was estimated on the basis of reasonable hydraulic conductivity values of the streambed material. Streambed material in the study area consists of silty sand and fractured bedrock with silty sand filling the fractures. Heath
(1983) estimated the horizontal hydraulic conductivity of silty sand to range from about 0.1 to $30 \mathrm{ft} / \mathrm{d}$. The bed of the Delaware River consists of about 6 in. of silty sand overlying fractured bedrock. The beds of the tributaries to the Delaware River in the study area typically have no sediments overlying the bedrock. Consequently, the bed thickness is greater and the streambed conduct ${ }^{\top}$ nce (the product of the vertical hydraulic conductivity of the streambed and the area of the stream within a model cell divided by the thickness of the streambed) is lower in the river than in the tributaries. The best model calibration was achieved when the vertical hydraulic conducti'ity of all streambed material was set at $0.5 \mathrm{ft} / \mathrm{d}$, th thickness of the riverbed was set at $1 \mathrm{ft}$, and the thickness of the beds of the tributaries was set at $0.1 \mathrm{ft}$.

\section{Areal Recharge}

Effective areal recharge rates were estimated from stream base-flow data collected in the model area. These data are summarized in table 3. Areal recharge is affected by land use. Agricultural, residential, commercial, and industrial areas may all receive different amourts of recharge because each has different proportiors of impermeable cover and is subject to different irrigation practices. The predominant land use in each model cell and in each drainage basin was determined by means of data in a geographic information system (GIS) coverage. By compering predominant land use and measured base flow in each drainage basin, recharge rates were estimated to be $11 \mathrm{in} / \mathrm{yr}$ in agricultural and undeveloped $\mathrm{c}$ reas and $5 \mathrm{in} / \mathrm{yr}$ in residential, commercial, and industrial areas. As a comparison, average annual precipitation measured at Trenton, New Jersey, is 42 inches.

\section{Specific Yield and Storage Coefficient}

Specific yield is used to define the storage capacity of model layer 1 , the weathered zone. which is assumed to be unconfined. Specific yield is defined as the ratio of the volume of water that 
Table 3. Base flow and ground-water runoff in streams at or near the Naval Air Warfare Center, West Trentcn, New Jersey $\left[\mathrm{mi}^{2}\right.$, square miles; $\mathrm{ft}^{3} / \mathrm{s}$, cubic feet per second; in/yr, inches per year; $\mathrm{nm}$, not measured]

\begin{tabular}{|c|c|c|c|c|c|c|}
\hline $\begin{array}{l}\text { Station } \\
\text { number }\end{array}$ & $\begin{array}{l}\text { Location of station } \\
\text { (see fig. 1) }\end{array}$ & $\begin{array}{l}\text { Predominant } \\
\text { land use(s) }\end{array}$ & $\begin{array}{l}\text { Drainage } \\
\text { area } \\
\left(\mathrm{mi}^{2}\right)\end{array}$ & $\begin{array}{l}\text { Date of } \\
\text { measure- } \\
\text { ment }\end{array}$ & $\begin{array}{l}\text { Base } \\
\text { flow } \\
\left(\mathrm{ft}^{3} / \mathrm{s}\right)\end{array}$ & $\begin{array}{c}\text { Mean } \\
\text { ground- } \\
\text { water } \\
\text { runoff at } \\
\text { station } \\
\text { (in/yr) }\end{array}$ \\
\hline \multirow[t]{5}{*}{1} & \multirow{5}{*}{$\begin{array}{l}\text { Mouth of unnamed tribu- } \\
\text { tary to the Delaware and } \\
\text { Raritan Feeder Canal at } \\
\text { Wilburtha, N.J. (unnamed } \\
\text { stream 3) }\end{array}$} & \multirow{5}{*}{$\begin{array}{l}\text { Agricultural } \\
\text { Residential }\end{array}$} & \multirow[t]{5}{*}{0.29} & $08 / 20 / 96$ & 0.383 & \multirow[t]{5}{*}{10} \\
\hline & & & & $05 / 16 / 97$ & .0027 & \\
\hline & & & & $09 / 04 / 97$ & .00004 & \\
\hline & & & & $09 / 29 / 98$ & 0 & \\
\hline & & & & $05 / 17 / 00$ & .25 & \\
\hline \multirow[t]{5}{*}{2} & \multirow{5}{*}{$\begin{array}{l}\text { Villa Victoria Brook, near } \\
\text { mouth, at Villa Victoria } \\
\text { Academy }\end{array}$} & \multirow{5}{*}{$\begin{array}{l}\text { Agricultural } \\
\text { Residential }\end{array}$} & \multirow[t]{5}{*}{1.10} & $08 / 20 / 96$ & .492 & \multirow[t]{5}{*}{5.6} \\
\hline & & & & $05 / 16 / 97$ & .829 & \\
\hline & & & & $09 / 04 / 97$ & .313 & \\
\hline & & & & $09 / 29 / 98$ & .160 & \\
\hline & & & & $05 / 17 / 00$ & .489 & \\
\hline \multirow[t]{5}{*}{3} & \multirow{5}{*}{$\begin{array}{l}\text { West Branch of Gold Run, } \\
\text { just west of Naval Air } \\
\text { Warfare Center }\end{array}$} & \multirow[t]{5}{*}{ Industrial } & \multirow[t]{5}{*}{.21} & $08 / 20 / 96$ & 0 & \multirow[t]{5}{*}{0} \\
\hline & & & & $05 / 16 / 97$ & 0 & \\
\hline & & & & $09 / 04 / 97$ & 0 & \\
\hline & & & & $09 / 29 / 98$ & 0 & \\
\hline & & & & $05 / 17 / 00$ & 0 & \\
\hline \multirow[t]{5}{*}{4} & \multirow{5}{*}{$\begin{array}{l}\text { Gold Run below culverts } \\
\text { containing west and east } \\
\text { branches of Gold Run just } \\
\text { east of Naval Air Warfare } \\
\text { Center }\end{array}$} & \multirow[t]{5}{*}{ Industrial } & \multirow[t]{5}{*}{.50} & $08 / 20 / 96$ & .09 & \multirow[t]{5}{*}{4.0} \\
\hline & & & & $05 / 16 / 97$ & $\mathrm{~nm}$ & \\
\hline & & & & $09 / 04 / 97$ & .203 & \\
\hline & & & & 09/29/98 & $\mathrm{nm}$ & \\
\hline & & & & $05 / 17 / 00$ & $\mathrm{~nm}$ & \\
\hline \multirow[t]{5}{*}{5} & \multirow{5}{*}{$\begin{array}{l}\text { Gold Run upstream from } \\
\text { Tributary } 1\end{array}$} & \multirow[t]{5}{*}{ Industrial } & \multirow[t]{5}{*}{.93} & $08 / 20 / 96$ & .53 & \multirow[t]{5}{*}{5.2} \\
\hline & & & & $05 / 16 / 97$ & .457 & \\
\hline & & & & $09 / 04 / 97$ & .177 & \\
\hline & & & & $09 / 30 / 98$ & .082 & \\
\hline & & & & $05 / 17 / 00$ & .52 & \\
\hline \multirow[t]{5}{*}{6} & \multirow{5}{*}{$\begin{array}{l}\text { Tributary } 1 \text { to Gold Run at } \\
\text { mouth }\end{array}$} & \multirow{5}{*}{$\begin{array}{l}\text { Residential } \\
\text { Industrial }\end{array}$} & \multirow[t]{5}{*}{.11} & $08 / 20 / 96$ & .117 & \multirow[t]{5}{*}{12} \\
\hline & & & & $05 / 16 / 97$ & .16 & \\
\hline & & & & $09 / 04 / 97$ & .117 & \\
\hline & & & & $09 / 30 / 98$ & .009 & \\
\hline & & & & $05 / 17 / 00$ & $\mathrm{~nm}$ & \\
\hline
\end{tabular}


Table 3. Base flow and ground-water runoff in streams at or near the Naval Air Warfare Center, West Trentın, New Jersey-Continued

$\left[\mathrm{mi}^{2}\right.$, square miles; $\mathrm{ft}^{3} / \mathrm{s}$, cubic feet per second; in/yr, inches per year; $\mathrm{nm}$, not measured]

\begin{tabular}{|c|c|c|c|c|c|c|}
\hline $\begin{array}{l}\text { Station } \\
\text { number }\end{array}$ & $\begin{array}{l}\text { Location of station } \\
\text { (see fig. 1) }\end{array}$ & $\begin{array}{l}\text { Predominant } \\
\text { land use(s) }\end{array}$ & $\begin{array}{l}\text { Drainage } \\
\text { area } \\
\left(\mathbf{m i}^{2}\right)\end{array}$ & $\begin{array}{l}\text { Date of } \\
\text { measure- } \\
\text { ment }\end{array}$ & $\begin{array}{l}\text { Base } \\
\text { flow } \\
\left(\mathrm{ft}^{3} / \mathrm{s}\right)\end{array}$ & $\begin{array}{c}\text { Mean } \\
\text { ground- } \\
\text { water } \\
\text { runoff at } \\
\text { station } \\
\text { (in/yr) } \\
\end{array}$ \\
\hline \multirow[t]{5}{*}{7} & \multirow{5}{*}{$\begin{array}{l}\text { Gold Run upstream from } \\
\text { Tributary } 2\end{array}$} & \multirow{5}{*}{$\begin{array}{l}\text { Industrial } \\
\text { Commercial }\end{array}$} & \multirow[t]{5}{*}{1.36} & $08 / 20 / 96$ & 0.740 & \multirow[t]{5}{*}{5.3} \\
\hline & & & & $05 / 16 / 97$ & .918 & \\
\hline & & & & $09 / 04 / 97$ & .348 & \\
\hline & & & & $09 / 30 / 98$ & .105 & \\
\hline & & & & $05 / 17 / 00$ & $\mathrm{~nm}$ & \\
\hline \multirow[t]{5}{*}{8} & \multirow{5}{*}{$\begin{array}{l}\text { Tributary } 2 \text { to Gold Run at } \\
\text { mouth }\end{array}$} & \multirow[t]{5}{*}{ Commercial } & \multirow[t]{5}{*}{.39} & $08 / 20 / 96$ & .176 & \multirow[t]{5}{*}{3.3} \\
\hline & & & & $05 / 16 / 97$ & .112 & \\
\hline & & & & $09 / 04 / 97$ & .088 & \\
\hline & & & & $09 / 04 / 98$ & 0 & \\
\hline & & & & $05 / 17 / 00$ & $\mathrm{~nm}$ & \\
\hline \multirow[t]{5}{*}{9} & \multirow{5}{*}{$\begin{array}{l}\text { Gold Run just upstream } \\
\text { from Delaware and Rari- } \\
\text { tan Feeder Canal }\end{array}$} & \multirow{5}{*}{$\begin{array}{l}\text { Commercial } \\
\text { Undeveloped } \\
\text { Residential }\end{array}$} & \multirow[t]{5}{*}{1.98} & $08 / 20 / 96$ & 1.44 & \multirow[t]{5}{*}{7.1} \\
\hline & & & & $05 / 16 / 97$ & 1.51 & \\
\hline & & & & $09 / 04 / 97$ & .668 & \\
\hline & & & & $09 / 04 / 98$ & .227 & \\
\hline & & & & $05 / 17 / 00$ & 1.30 & \\
\hline \multirow[t]{5}{*}{10} & \multirow{5}{*}{$\begin{array}{l}\text { West Branch of Sha- } \\
\text { bakunk Creek at Olden } \\
\text { Avenue }\end{array}$} & \multirow{5}{*}{$\begin{array}{l}\text { Residential } \\
\text { Commercial }\end{array}$} & \multirow[t]{5}{*}{2.74} & $08 / 20 / 97$ & $\mathrm{~nm}$ & \multirow[t]{5}{*}{5.5} \\
\hline & & & & $05 / 16 / 97$ & 2.04 & \\
\hline & & & & $09 / 04 / 97$ & 1.28 & \\
\hline & & & & $09 / 29 / 98$ & .153 & \\
\hline & & & & $05 / 17 / 00$ & .978 & \\
\hline
\end{tabular}


can be drained from a rock to the volume of the rock. In the calibrated model, the specific yield of the weathered zone was estimated to be 0.15 .

The storage term used in the model for layers below the weathered zone is storage coefficient, which is specific storage of the aquifer multiplied by the thickness of the aquifer. Specific storage is the amount of water released from or taken into storage per unit volume of aquifer per unit change in head. Rima and others (1962) found that the storage coefficient of the upper part of the Stockton Formation in southeastern Pennsylvania is about $1.37 \times 10^{-4}$. Because the water-bearing properties of the Lockatong Formation are lower than that of the Stockton Formation, the storage coefficient of the Lockatong Formation was assumed to be less than $10^{-4}$. During model calibration, specific storage was set at $4.0 \times 10^{-5}$ in all units below the weathered zone. Specific storage was multiplied by the thickness of each model cell to determine the storage coefficient of each cell.

\section{Simulation of Discharge Features}

Features in the model area that allow water to leave the ground-water system include streams and pumped wells. These features were included in the model by use of the "River" and "Wells" modules of MODFLOW.

\section{Streams}

Streams in the model area include the Delaware River, Villa Victoria Brook, Gold Run, unnamed streams 1,2, and 3, and West Branch Shabakunk Creek (fig. 1). The position of these streams relative to the model grid was determined by overlaying the model grid on topographic maps by means of a GIS. Field inspection of all streams except Gold Run indicate that the start of flow of each stream during base flow actually is about $1,500 \mathrm{ft}$ upstream from the mapped start of flow. The start of visible flow of Gold Run is at a point east of and across Parkway Avenue from the NAWC property at base-flow measurement site 4 (fig. 1). Historical maps (New Jersey Department of Environmental Protection, 1913) show that before the NAWC began operations in the mid-1950's, the start of flow of Gold Run was approximately $2(? \mathrm{ft}$ upstream from base-flow measurement site 3 (fig. 1). Since the early 1940 's, a culvert under Park'vay Avenue accepts ground-water discharge between base-flow measurement sites 3 and 4 (Lacombe, 2000). In the model, stream cells represent the culvert and the 200 - $\mathrm{ft}$ reach above measurement site 3.

The streams were simulated with the "River" module of MODFLOW. This module treats the streams as head-dependent boundaries. McDonald and Harbaugh (1988) define the flo'v between a stream and the aquifer in a given model cell according to the equation

$$
Q=\frac{K_{S} L W}{m_{s}}(h r i v-h a q)
$$

where

$Q$ is the flow between the stream and the aquifer, taken as positive if it is directed into the aquifer, in cubic feet per day;

$K_{\mathrm{s}}$ is the vertical hydraulic conductivity of the streambed material, in feet per day;

$L$ is the length of the reach within th 9 given model cell, in feet;

$W$ is the width of the stream, in feet;

$m_{\mathrm{s}}$ is the thickness of the streambed, in feet;

hriv is the head in the stream, in feet; and

haq is the head in the aquifer, in feet.

The length and width of the Delaware River and the length of the smaller streams within each model cell were estimated by intersecting GIS stream coverages with model-grid coverages. The width of the smaller streams was estimated at $2 \mathrm{ft}$ on the basis of measurements at a few representative sites. The thickness of the streambeds of the river and its tributaries was set at $1.0 \mathrm{ft}$ and $0.1 \mathrm{ft}$, respectively. 
The head in the stream at each model cell containing a stream was determined by (1) using topographic maps to determine the altitude of streams at points where topographic contours cross streams and then (2) interpolating between those points.

\section{Pumped Wells}

Most of the model simulations made as a part of this study included pumpage at one or more well. All of these wells are open to only one bedding unit except for well BRP1, which is open to the bottom $4 \mathrm{ft}$ of the weathered zone (model layer 1; see fig. 6), all $21 \mathrm{ft}$ of bedding unit 20 (model layer 22), and 15 of the $60 \mathrm{ft}$ of bedding unit 19 (model layer 23). In the model run in which well BRP1 was pumped, all of the pumpage was applied to bedding unit 20 because the well is open to only small parts of the adjacent bedding units.

\section{Calibration}

Measured ground-water levels and base flow in Villa Victoria Brook, unnamed stream 3, Gold Run, and West Branch Shabakunk Creek were used in calibrating the steady-state model. Measured drawdown was used to calibrate the transient model. The initial estimates of hydrologic parameters described in previous sections were adjusted within reasonable limits until 65 percent of simulated water levels were within $5 \mathrm{ft}$ of measured water levels; simulated base flow at each measurement site was within 17 percent of the mean measured base flow at the site; and 70 percent of simulated drawdowns were within $1 \mathrm{ft}$ of measured drawdown.

\section{Static Ground-Water Levels}

Ground-water levels measured at the NAWC on May 18,2000 , and at other wells in the model area on May 26, 2000, were used in calibrating the steady-state model. When the measurements at the NAWC were made on May 18, the six wells in recovery-well network I had been pumped almost continuously at about constant rates for at least 1 month preceding the measurements. No rainfall was recorded at a precipitationmeasurement site $9 \mathrm{mi}$ north of the NAWC from
May 15 through 18, 2000 (National Oceanic and Atmospheric Administration, 2000). Althougl 0.88 in. of precipitation was recorded on May 14, waterlevel data from continuous recorders at the NAWC indicate that water levels had returned to static levels. Therefore, water levels were static or rearly so when they were measured on May 18,200 .). In contrast, water levels measured at locations outside of the NAWC on May 26 probably were not static. Rainfall totaled 3.35 in. during May 19 through 25. At eight wells where water levels were measured on May 18 and again on May 26, the water level rose between 1.21 and $9.99 \mathrm{ft}$ during the 8-day period between the two measurements. In order to use the water-level data measured on the later date $\mathrm{fc}^{\text {r }}$ model calibration, the water levels measured on May 26 were decreased by $2.48 \mathrm{ft}$ (the median change in water levels at the eight wells measured both days). The measured and simulated water levels are listed in table 4 . Of the 89 simulated water levels in wells at the NAWC, 86 are within $10 \mathrm{ft}$ of the measured water levels, and 63 are within $5 \mathrm{ft}$. The average absolute difference between simulated and measured water levels at the NAWC is $4.32 \mathrm{ft}$.

At the 19 wells outside the NAWC property, 15 of the simulated water levels are vithin $10 \mathrm{ft}$ of the adjusted measured water levels, and 7 are within $5 \mathrm{ft}$. Most of the hydrogeologicframework data and hydraulic-conductivity data used to determine hydraulic parameters for the entire model area were collected at the NAWC site. If the estimated hydraulic parameters for ares s outside of the NAWC differ greatly from actral values, the result could be poorer agreement between measured and simulated water levels in wells outside of the NAWC, as opposed to wells at the NAWC.

Some of the wells used for calibration are open to more than one bedding unit. In this case, the following guidelines were used to arrive at the simulated head in the well. If the well opening straddles two or more bedding units, then the average water level in all units that are at least half included by the well opening was used as the simulated water level. If the well opening straddles two units but does not include more than half the thickness of either one, then the well was ass'gned to the layer containing the greater part of the open interval of the well. 
Table 4. Measured and simulated water levels in wells at and near the Naval Air Warfare Center, West Trenton, New Jersey, May 18 and 26, 2000

(Water levels are referenced to the National Geodetic Vertical Datum of 1929; --, no data; WZ, Weathered Zone; L, Lockatong; S, Stockt?n; R, water level recorded by continuous water-level recorder on $5 / 26 / 00$ at 1200 noon; $P$, pumped well; adj, adjusted to approximate $5 / 18 / 00$ conditions by subtracting 2.48 feet from water level measured on 5/26/00]

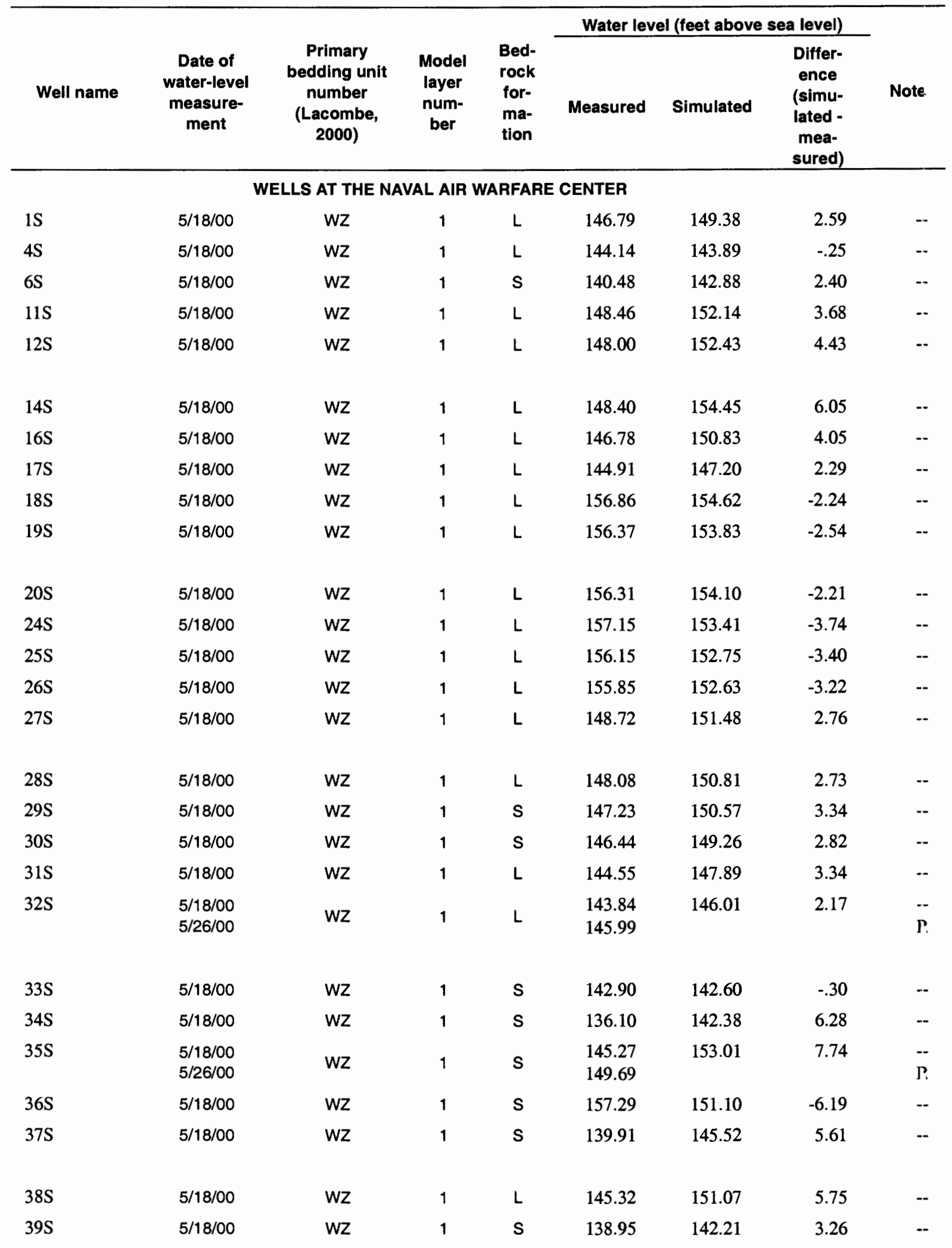


Table 4. Measured and simulated water levels in wells at and near the Naval Air Warfare Center, West Trention, New Jersey, May 18 and 26, 2000--Continued

\{Water levels are referenced to the National Geodetic Vertical Datum of 1929; --, no data; WZ, Weathered Zone; L, Lockatong; S, Stockton; R, water level recorded by continuous water-level recorder on $5 / 26 / 00$ at 1200 noon; $P$, pumped well; adj, adjusted to approximate $5 / 18 / 00$ conditions by subtracting 2.48 feet from water level measured on 5/26/00]

\begin{tabular}{|c|c|c|c|c|c|c|c|c|}
\hline \multirow[b]{2}{*}{ Well name } & \multirow[b]{2}{*}{$\begin{array}{c}\text { Date of } \\
\text { water-level } \\
\text { measure- } \\
\text { ment }\end{array}$} & \multirow[b]{2}{*}{$\begin{array}{c}\text { Primary } \\
\text { bedding unit } \\
\text { number } \\
\text { (Lacombe, } \\
\text { 2000) }\end{array}$} & \multirow[b]{2}{*}{$\begin{array}{l}\text { Model } \\
\text { layer } \\
\text { num- } \\
\text { ber }\end{array}$} & \multirow[b]{2}{*}{$\begin{array}{l}\text { Bed- } \\
\text { rock } \\
\text { for- } \\
\text { ma- } \\
\text { tion }\end{array}$} & \multicolumn{3}{|c|}{ Water level (feet above sea level) } & \multirow[b]{2}{*}{ Note } \\
\hline & & & & & Measured & Simulated & $\begin{array}{c}\text { Differ- } \\
\text { ence } \\
\text { (simu- } \\
\text { lated - } \\
\text { mea- } \\
\text { sured) }\end{array}$ & \\
\hline $40 \mathrm{~S}$ & $5 / 18 / 00$ & WZ & 1 & $L$ & 142.81 & 144.06 & 1.25 & -- \\
\hline $42 S$ & $5 / 18 / 00$ & WZ & 1 & s & 142.89 & 150.24 & 7.35 & -- \\
\hline 11MW1 & $5 / 18 / 00$ & WZ & 1 & s & 145.26 & 148.38 & 3.12 & - \\
\hline $12 \mathrm{MWl}$ & $5 / 18 / 00$ & WZ & 1 & $s$ & 145.95 & 151.20 & 5.25 & - \\
\hline 35MW1 & $5 / 18 / 00$ & Wz & 1 & L & 157.33 & 154.33 & -3.00 & -- \\
\hline $35 \mathrm{MW} 2$ & $5 / 18 / 00$ & wz & 1 & L & 156.79 & 153.93 & -2.86 & -- \\
\hline BRP1 & $5 / 18 / 00$ & L20 & 22 & $L$ & 144.57 & 147.34 & 2.77 & -- \\
\hline BRP2 & $5 / 18 / 00$ & L17 & 25 & $L$ & 141.51 & 143.09 & 1.58 & -- \\
\hline BRP3 & $\begin{array}{l}5 / 18 / 00 \\
5 / 26 / 00\end{array}$ & L19 & 23 & $L$ & $\begin{array}{l}143.90 \\
146.28\end{array}$ & 143.57 & -.33 & $\begin{array}{l}-- \\
R\end{array}$ \\
\hline 2BR & $5 / 18 / 00$ & L20 & 22 & $L$ & 147.69 & 152.17 & 4.48 & -- \\
\hline 3BR & $5 / 18 / 00$ & $S 15$ & 27 & $s$ & 145.18 & 150.24 & 5.05 & -- \\
\hline 4BR & $5 / 18 / 00$ & L17 & 25 & $L$ & 142.28 & 145.73 & 3.45 & -- \\
\hline $5 B R$ & $5 / 18 / 00$ & L15 & 27 & $L$ & 142.20 & 144.18 & 1.98 & - \\
\hline 6BR & $5 / 18 / 00$ & s11 & 31 & $s$ & 140.33 & 141.22 & .89 & -- \\
\hline 7BR & $5 / 18 / 00$ & L18 & 24 & $L$ & 141.74 & 144.68 & 2.94 & -- \\
\hline $8 \mathrm{BR}$ & $5 / 18 / 00$ & L22 & 20 & $L$ & 146.41 & 148.95 & 2.54 & -- \\
\hline 9BR & $5 / 18 / 00$ & L23 & 19 & $L$ & 149.43 & 150.92 & 1.49 & -- \\
\hline 10BR & $5 / 18 / 00$ & -- & 18 & $L$ & 158.21 & 155.98 & -2.23 & -- \\
\hline 11BR & $5 / 18 / 00$ & L21 & 21 & $L$ & 147.78 & 154.23 & 6.45 & -- \\
\hline 12BR & $\begin{array}{l}5 / 18 / 00 \\
5 / 26 / 00\end{array}$ & L22 & 20 & $L$ & $\begin{array}{l}147.43 \\
148.64\end{array}$ & 154.53 & 7.10 & $\bar{R}$ \\
\hline 13BR & $5 / 18 / 00$ & L23 & 19 & $L$ & 149.84 & 152.85 & 3.01 & -- \\
\hline 14BR & $5 / 18 / 00$ & L22 & 20 & $L$ & 149.29 & 153.74 & 4.45 & -- \\
\hline 15BR & $5 / 18 / 00$ & L19 & 23 & $L$ & 131.26 & 138.07 & 6.81 & $\mathbf{P}$ \\
\hline 16BR & $5 / 18 / 00$ & L19 & 23 & $L$ & 140.32 & 146.61 & 6.29 & -- \\
\hline $17 \mathrm{BR}$ & $5 / 18 / 00$ & L19 & 23 & L & 142.77 & 146.62 & 3.85 & -- \\
\hline $18 \mathrm{BR}$ & $5 / 18 / 00$ & -- & 15 & $L$ & 160.29 & 161.72 & 1.43 & - \\
\hline 20BR & $5 / 18 / 00$ & L17 & 25 & $L$ & 137.18 & 139.68 & 2.50 & $\mathbf{P}$ \\
\hline 21BR & $5 / 18 / 00$ & L23 & 19 & $L$ & 155.87 & 152.27 & -3.60 & -- \\
\hline
\end{tabular}


Table 4. Measured and simulated water levels in wells at and near the Naval Air Warfare Center, West Trentcn, New Jersey, May 18 and 26, 2000--Continued

\{Water levels are referenced to the National Geodetic Vertical Datum of $1929 ;--$, no data; WZ, Weathered Zone; L, Lockatong; S, Stockton; R, water level recorded by continuous water-level recorder on 5/26/00 at 1200 noon; $P$, pumped well; adj, adjusted to approximate 5/18/00 conditions ty subtracting 2.48 feet from water level measured on 5/26/00]

\begin{tabular}{|c|c|c|c|c|c|c|c|c|}
\hline \multirow[b]{2}{*}{ Well name } & \multirow[b]{2}{*}{$\begin{array}{c}\text { Date of } \\
\text { water-level } \\
\text { measure- } \\
\text { ment }\end{array}$} & \multirow[b]{2}{*}{$\begin{array}{c}\text { Primary } \\
\text { bedding unit } \\
\text { number } \\
\text { (Lacombe, } \\
\text { 2000) }\end{array}$} & \multirow[b]{2}{*}{$\begin{array}{l}\text { Model } \\
\text { layer } \\
\text { num- } \\
\text { ber }\end{array}$} & \multirow[b]{2}{*}{$\begin{array}{l}\text { Bed- } \\
\text { rock } \\
\text { for- } \\
\text { ma- } \\
\text { tion }\end{array}$} & \multicolumn{3}{|c|}{ Water level (feet above sea level) } & \multirow[b]{2}{*}{ Not: } \\
\hline & & & & & Measured & Simulated & $\begin{array}{l}\text { Differ- } \\
\text { ence } \\
\text { (simu- } \\
\text { lated- } \\
\text { mea- } \\
\text { sured) }\end{array}$ & \\
\hline 22BR & $5 / 18 / 00$ & $S 13$ & 29 & s & 136.80 & 116.97 & -19.83 & $\mathbf{P}$ \\
\hline 23BR & $5 / 18 / 00$ & L16 & 26 & $L$ & 142.01 & 145.64 & 3.63 & -- \\
\hline 24BR & $5 / 18 / 00$ & L16 & 26 & $\mathrm{~L}$ & 141.98 & 144.97 & 2.99 & - \\
\hline 26BR & $5 / 18 / 00$ & L15 & 27 & L & 143.36 & 143.90 & .54 & - \\
\hline 27BR & $5 / 18 / 00$ & L13 & 29 & $\mathbf{L}$ & 140.65 & 144.46 & 3.81 & -- \\
\hline 28BR & $5 / 18 / 00$ & S12 & 30 & s & 141.10 & 137.61 & -3.49 & - \\
\hline 29BR & $5 / 18 / 00$ & L21 & 21 & L & 144.55 & 147.93 & 3.38 & -. \\
\hline 30BR & $5 / 18 / 00$ & L18 & 24 & L & 139.49 & 146.36 & 6.87 & -- \\
\hline 31BR & $5 / 18 / 00$ & L15 & 27 & L & 141.32 & 147.25 & 5.93 & - \\
\hline 32BR & $5 / 18 / 00$ & S14 & 28 & $s$ & 145.08 & 151.92 & 6.84 & -- \\
\hline 33BR & $\begin{array}{l}5 / 18 / 00 \\
5 / 26 / 00\end{array}$ & L21 & 21 & L & $\begin{array}{l}144.32 \\
146.57\end{array}$ & 146.16 & 1.84 & $\bar{R}$ \\
\hline 34BR & $5 / 18 / 00$ & L15 & 27 & L & 143.09 & 144.80 & 1.71 & -- \\
\hline 35BR & $5 / 18 / 00$ & -- & 32 & $s$ & 143.25 & 142.43 & -.82 & -- \\
\hline 36BR & $5 / 18 / 00$ & L19 & 23 & L & 141.26 & 144.63 & 3.37 & -- \\
\hline 37BR & $5 / 18 / 00$ & S11 & 31 & s & 137.84 & 144.90 & 7.06 & -- \\
\hline 38BR & $\begin{array}{l}5 / 18 / 00 \\
5 / 26 / 00\end{array}$ & L19 & 23 & L & $\begin{array}{l}134.59 \\
144.58\end{array}$ & 147.00 & 12.41 & $\overline{\mathrm{R}}$ \\
\hline 39BR & $5 / 18 / 00$ & L15 & 27 & L & 142.06 & 146.45 & 4.39 & \\
\hline 40BR & $\begin{array}{l}5 / 18 / 00 \\
5 / 26 / 00\end{array}$ & L18 & 24 & L & $\begin{array}{l}142.41 \\
145.00\end{array}$ & 144.34 & 1.93 & $\overline{\mathrm{R}}$ \\
\hline $41 \mathrm{BR}$ & $5 / 18 / 00$ & L19 & 23 & $L$ & 135.00 & 144.51 & 9.51 & $P$ \\
\hline 42BR & $5 / 18 / 00$ & L13 & 29 & L & 142.07 & 146.24 & 4.17 & -- \\
\hline 45BR & $5 / 18 / 00$ & L19 & 23 & $\mathrm{~L}$ & 111.98 & 119.44 & 7.46 & $\mathrm{P}$ \\
\hline 47BR & $5 / 18 / 00$ & $w z$ & 1 & L & 144.45 & 147.21 & 2.76 & -- \\
\hline 48BR & $5 / 18 / 00$ & L17 & 25 & L & 103.30 & 137.31 & -34.01 & $P$ \\
\hline 49BR & $5 / 18 / 00$ & L17 & 25 & L & 139.09 & 147.34 & 8.25 & -- \\
\hline 50BR & $5 / 18 / 00$ & L17 & 25 & L & 146.88 & 151.78 & 4.90 & -- \\
\hline 51BR & $\begin{array}{l}5 / 18 / 00 \\
5 / 26 / 00\end{array}$ & L20 & 22 & L & $\begin{array}{l}146.00 \\
148.93\end{array}$ & 152.37 & 6.37 & $\bar{R}$ \\
\hline 52BR & $5 / 18 / 00$ & -- & 32 & L & 141.51 & 139.44 & -2.07 & -- \\
\hline
\end{tabular}


Table 4. Measured and simulated water levels in wells at and near the Naval Air Warfare Center, West Trenton, New Jersey, May 18 and 26, 2000--Continued

(Water levels are referenced to the National Geodetic Vertical Datum of 1929; --, no data; WZ, Weathered Zone; L, Lockatong; S, Stock ton; R, water level recorded by continuous water-level recorder on $5 / 26 / 00$ at 1200 noon; $P$, pumped well; adj, adjusted to approximate $5 / 18 / 00$ conditions by subtracting 2.48 feet from water level measured on $5 / 26 / 00$ ]

\begin{tabular}{|c|c|c|c|c|c|c|c|c|}
\hline \multirow[b]{2}{*}{ Well name } & \multirow[b]{2}{*}{$\begin{array}{c}\text { Date of } \\
\text { water-level } \\
\text { measure- } \\
\text { ment }\end{array}$} & \multirow[b]{2}{*}{$\begin{array}{c}\text { Primary } \\
\text { bedding unit } \\
\text { number } \\
(\text { Lacombe, } \\
2000)\end{array}$} & \multirow[b]{2}{*}{$\begin{array}{l}\text { Model } \\
\text { layer } \\
\text { num- } \\
\text { ber }\end{array}$} & \multirow[b]{2}{*}{$\begin{array}{l}\text { Bed- } \\
\text { rock } \\
\text { for- } \\
\text { ma- } \\
\text { tion }\end{array}$} & \multicolumn{3}{|c|}{ Water level (feet above sea level) } & \multirow[b]{2}{*}{ Not? } \\
\hline & & & & & Measured & Simulated & $\begin{array}{c}\text { Differ- } \\
\text { ence } \\
\text { (simu- } \\
\text { lated - } \\
\text { mea- } \\
\text { sured) }\end{array}$ & \\
\hline 53BR & $5 / 18 / 00$ & L19 & 23 & $\mathbf{L}$ & 140.91 & 147.80 & 6.89 & -- \\
\hline 54BR & $5 / 18 / 00$ & -- & 30 & $\mathbf{L}$ & 140.65 & 147.80 & 7.15 & -- \\
\hline 55BR & $5 / 18 / 00$ & L21 & 21 & $\mathbf{L}$ & 149.93 & 150.20 & .27 & -- \\
\hline $56 \mathrm{BR}$ & $5 / 18 / 00$ & L17 & 25 & $\mathbf{L}$ & 143.03 & 144.15 & 1.12 & - \\
\hline 57BR & $5 / 18 / 00$ & WZ & 1 & $\mathbf{L}$ & 144.06 & 145.85 & 1.79 & -- \\
\hline 58BR & $5 / 18 / 00$ & L18 & 24 & $L$ & 143.78 & 145.42 & 1.64 & -- \\
\hline 59BR & $5 / 18 / 00$ & L19 & 23 & $\mathbf{L}$ & 143.10 & 145.25 & 2.15 & -- \\
\hline \multicolumn{9}{|c|}{ WELLS OUTSIDE NAVAL AIR WARFARE CENTER PROPERTY } \\
\hline MW101 & $5 / 26 / 00$ & -- & 1 & $\mathbf{L}$ & 193.25 & 197.91 & 4.66 & $\operatorname{afj}$ \\
\hline Civil Defense & $5 / 26 / 00$ & -- & $18-24$ & $\mathrm{~L}$ & 104.32 & 70.09 & -34.23 & atj \\
\hline MW3 & $5 / 26 / 00$ & -- & 54 & S & 43.85 & 77.71 & 33.86 & $\mathrm{atj}$ \\
\hline MW4 & $5 / 26 / 00$ & -- & 1 & 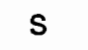 & 38.87 & 51.56 & 12.69 & $\mathrm{adj}$ \\
\hline NJM5 & $5 / 26 / 00$ & -- & 1 & $S$ & 61.57 & 51.03 & -10.54 & $\mathbf{a d j}$ \\
\hline NJM6 & $5 / 26 / 00$ & -- & 1 & $\mathrm{~s}$ & 67.28 & 76.31 & 9.03 & ali \\
\hline NJM7 & $5 / 26 / 00$ & -- & 1 & $S$ & 73.72 & 75.42 & 1.70 & ałj \\
\hline NJM11 & $5 / 26 / 00$ & -- & & $S$ & 67.12 & 63.93 & -3.19 & adi \\
\hline MW7-17 & $5 / 26 / 00$ & -- & 1 & $\mathrm{~S}$ & 117.86 & 114.50 & -3.36 & adi \\
\hline MW7-80 & $5 / 26 / 00$ & -- & 45 & $\mathrm{~s}$ & 115.00 & 113.87 & -1.13 & ali \\
\hline MW7-250 & $5 / 26 / 00$ & -- & 49 & $\mathrm{~s}$ & 103.76 & 111.03 & 7.27 & adi \\
\hline MW32-25 & $5 / 26 / 00$ & -- & 1 & $S$ & 93.10 & 98.56 & 5.46 & adj \\
\hline MW32-57 & $5 / 26 / 00$ & -- & 48 & $\mathrm{~s}$ & 92.57 & 98.23 & 5.66 & adj \\
\hline MW35-25 & $5 / 26 / 00$ & -- & 1 & $s$ & 102.07 & 104.21 & 2.14 & adj \\
\hline MW35-57 & $5 / 26 / 00$ & -- & 48 & $\mathrm{~s}$ & 101.96 & 103.24 & 1.28 & adj \\
\hline MW44-30 & $5 / 26 / 00$ & .. & 1 & $\mathrm{~s}$ & 108.52 & 114.41 & 5.89 & adj \\
\hline MW44-80 & $5 / 26 / 00$ & -- & 44 & $\mathrm{~s}$ & 104.31 & 113.79 & 9.48 & adj \\
\hline MW48-25 & $5 / 26 / 00$ & -- & 1 & $\mathrm{~s}$ & 94.48 & 103.18 & 8.70 & adj \\
\hline MW48-63 & $5 / 26 / 00$ & $-\cdot$ & 49 & $\mathrm{~s}$ & 94.18 & 104.02 & 9.84 & $\mathrm{adj}$ \\
\hline
\end{tabular}




\section{Base Flow to Streams}

The steady-state model simulating the scenario when recovery-well network I was operating also was calibrated to mean measured base flow in streams in the model area. Simulated base flow to streams was calculated by use of the computer program Zonebudget (Harbaugh, 1990). The measured and simulated base flow at the mouth of each stream is listed in table 5. All simulated base flows are within 17 percent of the mean measured base flow. Given the measurement error inherent in base-flow measurements, the model calibration is considered to be acceptable with regard to its simulation of ground-water discharge to streams.

\section{Drawdown During Short-Term Pumping}

The transient model was calibrated to drawdown that resulted from pumping three different wells for short periods of time. Well 1:BR was pumped at a rate of $25 \mathrm{gal} / \mathrm{min}$ for 72 hour: on April 8-11, 1993 (International Technology Corporation, 1994); well BRP1 was pumped at a rate of $12.5 \mathrm{gal} / \mathrm{min}$ for 48 hours on August 9-11, 1995; and well 5BR was pumped at a rate of $25 \mathrm{gal} /$ min for 42 hours on August 15-17, 1995 (EA Engineering, Science, and Technology, Inc., 1976). Measured drawdown in wells near these pumped wells ranges from 0 to $11.50 \mathrm{ft}$. Simulated drawdown at 70 percent of the wells are within $1 \mathrm{ft}$ of the measured drawdown. The difference betw'een measured and simulated drawdown ranges from 0.01 to $3.26 \mathrm{ft}$. The measured and simulated drawdowns for each well are listed in tables 6, 7, and 8.

Table 5. Measured and simulated base flow to streams at and near the Naval Air Warfare Center, West Trenton, New Jersey

$\left[\mathrm{ft}^{3} / \mathrm{s}\right.$, cubic feet per second; $\mathrm{ft}^{3} / \mathrm{d}$, cubic feet per day]

\begin{tabular}{|c|c|c|c|c|c|}
\hline \multirow{2}{*}{$\begin{array}{c}\text { Station } \\
\text { number } \\
\text { (shown on } \\
\text { figure 1) }\end{array}$} & \multirow[b]{2}{*}{$\begin{array}{c}\text { Location of } \\
\text { station }\end{array}$} & \multicolumn{2}{|c|}{ Mean measured base flow } & \multicolumn{2}{|c|}{ Simulated base flow } \\
\hline & & $\mathrm{ft}^{3} / \mathrm{s}$ & $\mathrm{ft}^{3} / \mathrm{d}$ & $\mathrm{ft}^{3} / \mathrm{d}$ & $\begin{array}{l}\text { Percent } \\
\text { of mea- } \\
\text { sured }\end{array}$ \\
\hline 1 & $\begin{array}{l}\text { Mouth of } \\
\text { unnamed tribu- } \\
\text { tary to the Dela- } \\
\text { ware and } \\
\text { Raritan Feeder } \\
\text { Canal at Wil- } \\
\text { burtha,N.J.(un- } \\
\text { named stream 3) }\end{array}$ & 0.103 & 8,900 & 9,010 & 101 \\
\hline 2 & $\begin{array}{l}\text { Villa Victoria } \\
\text { Brook, near } \\
\text { mouth, at Villa } \\
\text { Victoria Acad- } \\
\text { emy }\end{array}$ & .457 & 39,490 & 33,400 & 85 \\
\hline 9 & $\begin{array}{l}\text { Gold Run } 20 \\
\text { feet upstream } \\
\text { from Delaware } \\
\text { and Raritan } \\
\text { Feeder Canal }\end{array}$ & 1.029 & 88,910 & 73,500 & 83 \\
\hline 10 & $\begin{array}{l}\text { West Branch of } \\
\text { Shabakunk } \\
\text { Creek at Olden } \\
\text { Avenue }\end{array}$ & 1.113 & 96,160 & 97,590 & 101 \\
\hline
\end{tabular}


Table 6. Measured and simulated drawdown in wells at the Naval Air Warfare Center, West Trenton, New Jersey, after well 15BR had been pumped at 25 gallons per minute for 72 hours

\begin{tabular}{|c|c|c|c|c|c|c|}
\hline $\begin{array}{l}\text { Well } \\
\text { name }\end{array}$ & $\begin{array}{c}\text { Bedding } \\
\text { unit number } \\
\text { (Lacombe, } \\
\text { 2001) }\end{array}$ & $\begin{array}{c}\text { Model layer } \\
\text { number }\end{array}$ & $\begin{array}{c}\text { Bedrock } \\
\text { formation }\end{array}$ & $\begin{array}{l}\text { Measured } \\
\text { drawdown } \\
\text { (feet) }\end{array}$ & $\begin{array}{c}\text { Simulated } \\
\text { drawdown } \\
\text { (feet) }\end{array}$ & $\begin{array}{c}\text { Difference } \\
\text { between mea- } \\
\text { sured and } \\
\text { simulated } \\
\text { drawdown } \\
\text { (simulated - } \\
\text { measured, in } \\
\text { feet) }\end{array}$ \\
\hline BRP1 & L20 & 22 & Lockatong & 1.50 & 0.45 & -1.05 \\
\hline BRP2 & L17 & 25 & Lockatong & .87 & 1.12 & .25 \\
\hline BRP3 & L19 & 23 & Lockatong & 2.56 & 3.50 & .94 \\
\hline 4BR & L17 & 25 & Lockatong & .74 & .64 & -.10 \\
\hline $5 B R$ & L15 & 27 & Lockatong & .45 & .78 & .33 \\
\hline 6BR & $s 11$ & 31 & Stockton & .00 & .08 & .08 \\
\hline 7BR & L18 & 24 & Lockatong & 1.48 & 1.80 & .32 \\
\hline 8BR & L22 & 20 & Lockatong & .82 & .36 & -.48 \\
\hline 15BR & L19 & 23 & Lockatong & 11.50 & 12.80 & 1.30 \\
\hline 16BR & L19 & 23 & Lockatong & 1.52 & .37 & -1.15 \\
\hline 17BR & L19 & 23 & Lockatong & 1.12 & .52 & -.60 \\
\hline 20BR & L17 & 25 & Lockatong & .97 & .96 & -.01 \\
\hline 22BR & $S 13$ & 29 & Stockton & .00 & .05 & .05 \\
\hline $23 B R$ & L16 & 26 & Lockatong & .63 & .64 & .01 \\
\hline 24BR & L16 & 26 & Lockatong & .72 & 1.04 & .32 \\
\hline $25 \mathrm{BR}$ & L18 & 24 & Lockatong & 1.69 & 3.31 & 1.62 \\
\hline 26BR & L15 & 27 & Lockatong & .51 & .76 & .25 \\
\hline 27BR & L13 & 29 & Lockatong & .12 & .59 & .47 \\
\hline $28 B R$ & $\mathrm{~s} 12$ & 30 & Stockton & .00 & .05 & .05 \\
\hline 29BR & L21 & 21 & Lockatong & 1.99 & .57 & -1.42 \\
\hline 30BR & L18 & 24 & Lockatong & 1.19 & .59 & -.60 \\
\hline 33BR & L21 & 21 & Lockatong & 2.31 & .59 & -1.72 \\
\hline 34BR & L15 & 27 & Lockatong & .49 & .41 & -.08 \\
\hline 36BR & L19 & 23 & Lockatong & 2.95 & 2.54 & -.41 \\
\hline 38BR & 19 & 23 & Lockatong & 3.87 & .61 & -3.26 \\
\hline
\end{tabular}


Table 7. Measured and simulated drawdown in wells at the Naval Air Warfare Center, West Trenton, New Jersey, after well BRP1 had been pumped at 12.5 gallons per minute for $\mathbf{4 8}$ hours

\begin{tabular}{|c|c|c|c|c|c|c|}
\hline $\begin{array}{l}\text { Well } \\
\text { name }\end{array}$ & $\begin{array}{c}\text { Bedding } \\
\text { unit number } \\
\text { (Lacombe, } \\
\text { 2001) }\end{array}$ & $\begin{array}{c}\text { Model layer } \\
\text { number }\end{array}$ & $\begin{array}{l}\text { Bedrock } \\
\text { formation }\end{array}$ & $\begin{array}{c}\text { Measured } \\
\text { drawdown } \\
\text { (feet) }\end{array}$ & $\begin{array}{l}\text { Simulated } \\
\text { drawdown } \\
\text { (feet) }\end{array}$ & $\begin{array}{l}\text { Difference } \\
\text { between } \\
\text { measured } \\
\text { and simu- } \\
\text { lated draw- } \\
\text { down } \\
\text { (feet) }\end{array}$ \\
\hline $17 \mathrm{~S}$ & $\begin{array}{l}\text { Weathered } \\
\text { Zone }\end{array}$ & 1 & Lockatong & 0.67 & 0.56 & -0.11 \\
\hline 4BR & L17 & 25 & Lockatong & .40 & .62 & .22 \\
\hline 8BR & L22 & 20 & Lockatong & .91 & .35 & -.56 \\
\hline $16 \mathrm{BR}$ & L19 & 23 & Lockatong & 1.83 & 1.94 & .11 \\
\hline $17 \mathrm{BR}$ & L19 & 23 & Lockatong & 1.56 & 2.48 & .92 \\
\hline 23BR & L16 & 26 & Lockatong & .15 & .56 & .41 \\
\hline 29BR & L21 & 21 & Lockatong & 1.82 & .52 & -1.30 \\
\hline 30BR & L18 & 24 & Lockatong & .40 & 1.56 & 1.16 \\
\hline 38BR & L19 & 23 & Lockatong & 1.07 & 3.17 & 2.10 \\
\hline
\end{tabular}

Table 8. Measured and simulated drawdown in wells at the Naval Air Warfare Center, West Trenton, New Jersey, after well 5BR had been pumped at 25 gallons per minute for 42 hours

\begin{tabular}{|c|c|c|c|c|c|c|}
\hline $\begin{array}{l}\text { Well } \\
\text { name }\end{array}$ & $\begin{array}{c}\text { Bedding } \\
\text { unit number } \\
\text { (Lacombe, } \\
2001\end{array}$ & $\begin{array}{c}\text { Model layer } \\
\text { number }\end{array}$ & $\begin{array}{l}\text { Bedrock } \\
\text { formation }\end{array}$ & $\begin{array}{c}\text { Measured } \\
\text { drawdown } \\
\text { (feet) }\end{array}$ & $\begin{array}{c}\text { Simulated } \\
\text { drawdown } \\
\text { (feet) }\end{array}$ & $\begin{array}{c}\text { Difference } \\
\text { between } \\
\text { measured } \\
\text { and simu- } \\
\text { lated draw- } \\
\text { down } \\
\text { (feet) }\end{array}$ \\
\hline $32 S$ & $\begin{array}{l}\text { Weathered } \\
\text { Zone }\end{array}$ & 1 & Lockatong & 0.00 & 0.09 & 0.09 \\
\hline $41 S$ & $\begin{array}{l}\text { Weathered } \\
\text { Zone }\end{array}$ & 1 & Lockatong & .00 & .23 & .23 \\
\hline 4BR & L17 & 25 & Lockatong & 1.09 & .80 & -.29 \\
\hline 7BR & L18 & 24 & Lockatong & .73 & .93 & .20 \\
\hline 20BR & 17 & 25 & Lockatong & .91 & 1.95 & 1.04 \\
\hline 22BR & $\mathrm{S} 13$ & 29 & Stockton & .09 & .03 & -.06 \\
\hline 23BR & L16 & 26 & Lockatong & 2.03 & 1.22 & -.81 \\
\hline 24BR & L16 & 26 & Lockatong & 4.03 & 1.66 & -2.37 \\
\hline $25 B R$ & 18 & 24 & Lockatong & .00 & .92 & .92 \\
\hline 26BR & L15 & 27 & Lockatong & 7.05 & 4.22 & -2.83 \\
\hline 27BR & L13 & 29 & Lockatong & .27 & 2.87 & 2.60 \\
\hline 33BR & 21 & 21 & Lockatong & .00 & .25 & .25 \\
\hline
\end{tabular}




\section{Sensitivity Analysis}

The purpose of a sensitivity analysis is to quantify the uncertainty in the calibrated model. In the model used in this study, uncertainty is created by estimation of the horizontal and vertical hydraulic conductivity, storage coefficient, and recharge rates. A sensitivity analysis was performed by systematically changing the values of these parameters within hydrologically reasonable ranges. Although the model results also may be sensitive to boundary conditions, grid discretization, and spatial and temporal variations of parameters, sensitivity testing of these factors is impractical. Results of the sensitivity analysis are reported as the effects of the parameter changes on the simulated water levels, drawdown, and base flow to streams. Because an important use of the model is to test the effectiveness of recovery-well networks in capturing contaminated water (discussed in the section "Simulated ground-water flowpaths" later on), the effects of parameter changes on the number of contaminated well locations captured by recovery wells also were computed.

Results of the sensitivity analysis are shown in figure 7. Changes in hydraulic properties were applied equally to all model cells, and changes in recharge were applied equally to all active cells in layer 1 (fig. 4). These parameters were varied over the range from one-half to two times the calibrated value. The steady-state model was most sensitive to recharge and least sensitive to vertical hydraulic conductivity. The transient model was most sensitive to horizontal hydraulic conductivity and least sensitive to storage coefficient.

\section{Simulated Water Budget}

Simulated water budgets for three steadystate simulations are listed in table 9. One simulation represents a scenario when no wells are pumped at the NAWC; the other two simulations represent a scenario when recovery-well networks I and II are in operation. All three simulations include steady pumping of $3,850 \mathrm{ft}^{3} / \mathrm{d}$ at the Roller Bearing Corporation (RBC, fig. 1). The budgets, which were calculated with the computer program Zonebudget (Harbaugh, 1990), indicate that most of the water pumped at the NAWC recovery wells represents base flow diverted from Gold Run, Villa Victoria Brook, and West Branch Shabakunk Creek in noarly equal amounts. Small amounts of base flow als? are diverted from the Delaware River and unnamed streams 2 and 3.

The budgets also indicate that the change from recovery-well network I to recovery-well network II (a change from pumping well 41BR to pumping well BRP2) achieved the desired result of preventing contaminated ground water in the southwestern part of the NAWC property from discharging to Gold Run. The simulated water budgets indicate that recovery-well network $\mathrm{I}$ diverts about $290 \mathrm{ft}^{3} / \mathrm{d}$ more than network I dnes from Gold Run. Calculations of ground-water discharge to discrete reaches of Gold Run indicate that this additional diversion of $290 \mathrm{ft}^{3} / \mathrm{d}$ represents all of the ground water that was flowing from the southwestern part of the NAWC property into the uppermost 500-ft reach of Gold Run. The change from pumping well 41BR to pumping well BPP2 also causes $290 \mathrm{ft}^{3} / \mathrm{d}$ more ground water from the NAWC property to discharge to West Branch Shabakunk Creek. This additional discharge to West Branch Shabakunk Creek probably is not a threat to the water quality in the creek for two reasons: (1) ground water flowing from the NAWC property to the West Branch of Shabakunk Creek originates in and flows through the eastern part of the NAWC property, which is far less contaminated than the southwestern part of the property (Lacombe, 2000), and (2) the distance from the NAWC property to the nearest point of the West Branch of Shabakunk Creek is approximately 3,000 ft, whereas Gold Run flows through NA WC property.

Most of the water that enters the weatl ored zone (model layer 1) as recharge flows downward into the competent bedrock layers (model layers 2 to 71). When no wells at NAWC are pumped, 78 percent of recharge flows into the competent bedrock; when recovery-well network I or II is pumped, 80 percent of recharge flows into the competent bedrock. Except for water that flows to pumped wells, all the water that flows downward into competent bedrock eventually flows back into the weathered zone and leaves the ground-water system as base flow to streams. 

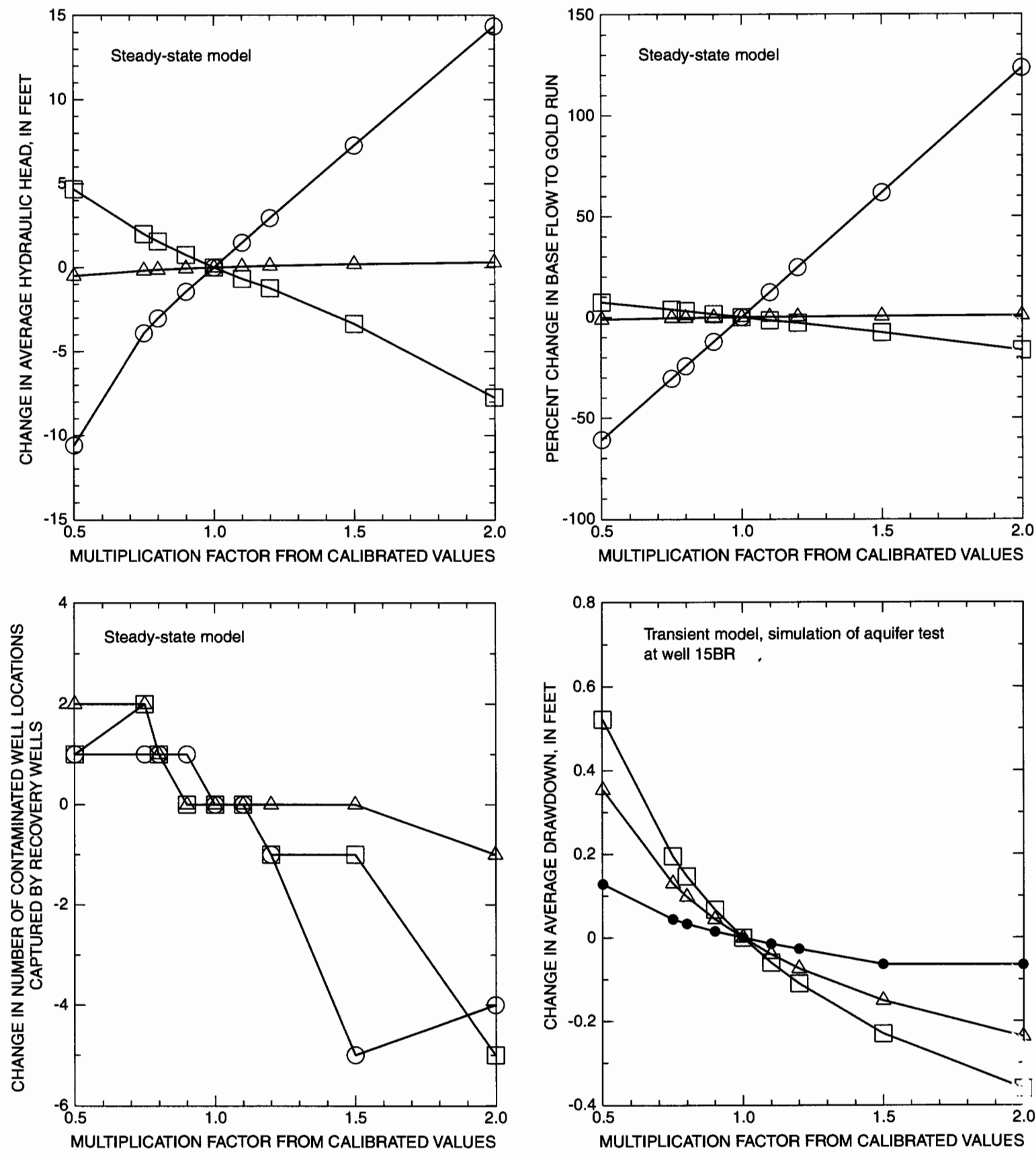

EXPLANATION

Aquifer horizontal hydraulic conductivity

$\triangle$ Aquifer vertical hydraulic conductivity

Recharge

- Storage coefficient

Figure 7. Sensitivity of simulated ground-water levels, base flow, drawdown, and capture of contaminated water by recovery wells to variations in the values of model parameters, Naval Air Warfare Center, West Trenton, New Jersey. 
Table 9. Simulated water budget at and near the Naval Air Warfare Center, West Trenton, New Jersey [ft $\mathrm{ft}^{3} / \mathrm{d}$, cubic feet per day; na, not applicable]

\begin{tabular}{|c|c|c|c|c|c|}
\hline & \multirow[b]{2}{*}{$\begin{array}{l}\text { Budget with no } \\
\text { recovery wells } \\
\text { pumping } \\
\left(\mathrm{ft}^{3} / \mathrm{d}\right)\end{array}$} & \multicolumn{2}{|c|}{$\begin{array}{c}\text { Budget with recovery well } \\
\text { network I operating }\end{array}$} & \multicolumn{2}{|c|}{$\begin{array}{l}\text { Budget with recovery-wr"l } \\
\text { network II operating }\end{array}$} \\
\hline & & $\begin{array}{l}\text { Flow } \\
\left(\mathrm{ft}^{3} / \mathrm{d}\right)\end{array}$ & $\begin{array}{l}\text { Base flow to } \\
\text { streams } \\
\text { diverted by } \\
\text { pumping at } \\
\text { NAWC } \\
\text { recovery- } \\
\text { wellnetwork } \\
\left(\mathrm{ft}^{3} / \mathrm{d}\right) \\
\end{array}$ & $\begin{array}{l}\text { Flow } \\
\left(\mathbf{f t}^{3} / \mathrm{d}\right)\end{array}$ & $\begin{array}{c}\text { Base flow to } \\
\text { streamr } \\
\text { diverted by } \\
\text { pumping at } \\
\text { NAWC } \\
\text { recover). } \\
\text { well network } \\
\left(\mathrm{ft}^{3} / \mathrm{d}\right) \\
\end{array}$ \\
\hline \multicolumn{6}{|l|}{$\begin{array}{l}\text { WATER INTO GROUND-WATER } \\
\text { SYSTEM }\end{array}$} \\
\hline Recharge & 348,320 & 348,320 & na & 348,320 & rq \\
\hline $\begin{array}{l}\text { Total into ground-water system } \\
\text { WATER OUT OF GROUND- } \\
\text { WATER SYSTEM }\end{array}$ & 348,320 & 348,320 & na & 348,320 & ra \\
\hline Base flow to Delaware River & 111,340 & 111,150 & 190 & 111,160 & $1 \varepsilon 0$ \\
\hline Base flow to Villa Victoria Brook & 36,710 & 33,400 & 3,310 & 33,390 & $3,3 \hat{2} \cap$ \\
\hline Base flow to Gold Run & 76,870 & 73,470 & 3,400 & 73,180 & 3,657 \\
\hline $\begin{array}{l}\text { Base flow to West Branch Sha- } \\
\text { bakunk Creek }\end{array}$ & 101,500 & 97,580 & 3,920 & 97,870 & $3,6 \cong ?$ \\
\hline Base flow to unnamed stream 1 & 1,180 & 1,180 & 0 & 1,180 & 0 \\
\hline Base flow to unnamed stream 2 & 7,790 & 7,780 & 10 & 7,780 & 10 \\
\hline Base flow to unnamed stream 3 & 9,040 & 9,000 & 40 & 9,000 & 10 \\
\hline $\begin{array}{l}\text { Flow to pumped wells (includes } \\
3,850 \mathrm{ft}^{3} / \mathrm{d} \text { at Roller Bearing Corpo- } \\
\text { ration) }\end{array}$ & 3,850 & 14,730 & na & 14,730 & rag \\
\hline Total out of ground-water system & 348,280 & 348,300 & 10,870 & 348,290 & $10,8 \%$ \\
\hline Difference (in - out) & 0 & 20 & na & 30 & ra \\
\hline
\end{tabular}

\section{Model Limitations}

Ground-water flow in fractured rocks is too complex to be succinctly simulated in a digital model. By making various necessary simplifying assumptions, however, one can construct a model that is capable of approximating flow through the fractured rock units.

The model used in this study is based on the assumption that the fault found at the NAWC property extends from the western to eastern model boundaries and that the fault permeability is very low throughout the entire fault zone. No data are available that indicate whether the lowpermeability assumption is valid throughout the entire model area. As a result of this assumption, the model simulates little ground-water flow across the fault anywhere in the modeled area. It is possible, however, that, if the fault is more permeable o* not present outside the NAWC area, large amount- of water could flow across the fault.

Another important assumption incorporated into the model is that ground-water flow is negligible at depths below $500 \mathrm{ft}$. If this assumption is flawed, then some of the simuleted flowpaths--especially paths beginning at deep wells--could be shallower than actual flowpat's, and the discharge points of these flowpaths could be inaccurately simulated by the model. 
The primary intended use of this model is to simulate ground-water flow and to estimate the effectiveness of two recovery-well networks at the NAWC. Most of the data used to calibrate the model are from the NAWC property. Therefore, the model should not be used to obtain detailed simulations of ground-water conditions outside of the NAWC area.

The model represents a porous medium, and the pores in this hydrogeologic setting consist of a network of fractures in three orientations that are at right angles to and interconnected with each other. Each model cell represents a volume of space that contains many fractures. The model calculates the flowpath from one cell to the next by simulating the net effect of all of the fractures in that interval. Therefore, the model cannot simulate flowpaths on a scale smaller than the cell size. In the NAWC area, this limitation means that flowpaths within areas smaller than a $25-\mathrm{ft}$ by $25-\mathrm{ft}$ square cannot be simulated.

Only advective movement of contaminants can be simulated by the model. The model does not simulate density-driven movement, dispersion, diffusion, dilution, or biological or chemical degradation of contaminants. The effects of densitydriven movement, dispersion, diffusion, and dilution widen the contaminant flowpaths, whereas degradation shortens them.

\section{Simulated Ground-Water}

\section{Flowpaths}

The flowpaths of contaminated water at the NAWC property under conditions when no recovery wells are pumped at the NAWC and when recovery-well networks I and II are used were estimated by means of the steady-state, groundwater-flow model. The purpose of the no-pumping simulation is to estimate the paths of contaminated ground water before either recovery-well network was installed. The purpose of the pumping simulations is to assess the effectiveness of the networks. The flowpaths and discharge points simulated by the model are approximate because of the limitations of modeling flow in fractured rock, as described in the preceding section; however, digital modeling provides a closer approximation to actual flowpaths than simpler methods, such ar water-level gradients, because many of the complexities caused by the fractured-rock hydrogeologic framework are incorporated into the digital model.

\section{Simulation 1--No Pumping}

This simulation depicts ground-water flow at times when no wells are pumping and reflects conditions before the recovery-well network I was installed. The results of this simulation are list $\gg d$ in table 10 and shown in figure 8 . The discharge point of water at and near each contaminated well at the NAWC is listed in table 10. For purposes of th is report, a well is contaminated if any sample collected from the well contained TCE, DCE, or vinyl chloride in concentrations above the $\mathrm{Ne}^{\mathrm{v}}$ Jersey maximum contaminant level, which is 1,10 , and 2 micrograms per liter, respectively.

In simulation 1 , ground water at and near most of the contaminated well sites in the southern part of the NAWC flows into the west branch of Gold Run. This water does not cross the fault; water from wells on the north side of the fault flows westsouthwest or southwest and discharges to the reach of Gold Run that is on the north side, whereas water at and near the nine wells on the south side of the fault remains on the south side and discharges into the Gold Run reach carried in the culvert beneath Parkway Avenue. Water at and near the three easternmost contaminated wells (14S, 11BR and 50 BR) flows generally east and discharges to West Branch Shabakunk Creek, also without crossirg the fault. Water at and near five contaminated we'ls in the northwesternmost part of the NAWC and one relatively deep well (56BR, $165 \mathrm{ft}$ deep) farther southwest flows generally southwest to Villa Victoria Brook, also remaining on the north side of the fault. Water at and near seven contaminated wells on the north side of the fault remains or the north side and discharges into the Delaware Fiver. Two of these wells (45BR and 46BR) are more than $200 \mathrm{ft}$ deep; their flowpaths remain deep and pass under Villa Victoria Brook before discharging into the river. The other five wells whose flowpaths discharge into the river (11S, 2BR, 49BR, 51BR, 
Table 10. Simulated discharge points of ground water at and near contaminated wells at the Naval Air Warfare Center (NAWC), West Trenton, New Jersey

[WZ, weathered zone; gal/min, gallons per minute; L, Lockatong; S, Stockton; --, no data)]

\begin{tabular}{|c|c|c|c|c|c|c|}
\hline \multirow[b]{2}{*}{ Well name } & \multirow[b]{2}{*}{$\begin{array}{c}\text { Primary } \\
\text { bedding } \\
\text { unit } \\
\text { (Lacombe, } \\
\text { 2000b) }\end{array}$} & \multirow[b]{2}{*}{$\begin{array}{l}\text { Model } \\
\text { layer } \\
\text { num- } \\
\text { ber }\end{array}$} & \multirow[b]{2}{*}{$\begin{array}{l}\text { Bed- } \\
\text { rock } \\
\text { for- } \\
\text { mat- } \\
\text { lon }\end{array}$} & \multicolumn{3}{|c|}{ Stream or pumped well where water discharges from ground-water syatem } \\
\hline & & & & $\begin{array}{c}\text { Simulation } 1 \\
\text { (no pumpage at the } \\
\text { NAWC) }\end{array}$ & $\begin{array}{c}\text { Simulation 2, } \\
\text { recovery-well } \\
\text { network I } \\
\text { (well 15BR pumped at } \\
15 \mathrm{gal} / \mathrm{min} ; 20 \mathrm{BR} \text { at } 8.5 \\
\mathrm{gal} / \mathrm{min} ; 22 \mathrm{BR} \text { at } 4.4 \\
\mathrm{gal} / \mathrm{min} ; 41 \mathrm{BR} \text { at } 10 \\
\mathrm{gal} / \mathrm{min} ; 45 \mathrm{BR} \text { at } 5.1 \\
\mathrm{gal} / \mathrm{min} ; 48 \mathrm{BR} \text { at } 13.5 \\
\mathrm{gal} / \mathrm{min} \text { ) }\end{array}$ & $\begin{array}{c}\text { Simulation 3, } \\
\text { recovery-wel } \\
\text { network II } \\
\text { (well 15BR pumped at } \\
15 \mathrm{gal} / \mathrm{min} \text {; 20BR } \$ \mathbf{8 . 5} \\
\mathrm{gal} / \mathrm{min} ; 22 \mathrm{BR} \text { at } 4.4 \\
\mathrm{gal} / \mathrm{min} \text {; 45BR at } 5.1 \\
\mathrm{gal} / \mathrm{min} \text {;8BR at } 13.5 \\
\mathrm{gal} / \mathrm{min} ; \mathrm{BRP2} \text { a* } 10 \\
\mathrm{gal} / \mathrm{min} \text { ) } \\
\end{array}$ \\
\hline $2 S$ & WZ & 1 & $\mathrm{~L}$ & Gold Run (west branch) & Well 15BR & Well 15BR \\
\hline $6 S$ & WZ & 1 & $\mathrm{~S}$ & Gold Run (west branch) & Well 22BR & Well 22BR \\
\hline $11 S$ & WZ & 1 & $\mathrm{~L}$ & Delaware River & Well 48BR & Well 48BR \\
\hline $12 S$ & WZ & 1 & $\mathrm{~L}$ & Gold Run (main stem) & Well 48BR & Well 48BR \\
\hline $13 S$ & WZ & 1 & $\mathrm{~L}$ & Gold Run (main stem) & Well 48BR & Well 48BR \\
\hline $14 S$ & WZ & 1 & $\mathrm{~L}$ & $\begin{array}{l}\text { West Branch Sha- } \\
\text { bakunk Creek }\end{array}$ & Well 48BR & Well 48BR \\
\hline $15 S$ & WZ & 1 & $\mathrm{~L}$ & Gold Run (west branch) & Well 15BR & Well 15BR \\
\hline $24 S$ & WZ & 1 & $\mathbf{L}$ & Villa Victoria Brook & Well 15BR & Well 15BR \\
\hline $25 S$ & $\mathrm{WZ}$ & 1 & $\mathrm{~L}$ & Villa Victoria Brook & Well 15BR & Well 15BR \\
\hline $28 S$ & WZ & 1 & $\mathrm{~L}$ & Gold Run (west branch) & Well 48BR & Well 48BR \\
\hline $31 S$ & WZ & 1 & $\mathrm{~L}$ & Gold Run (west branch) & Well 48BR & Well 48BR \\
\hline $37 \mathrm{~S}$ & WZ & 1 & $S$ & Gold Run (west branch) & Gold Run (west branch) & Gold Run (west branch) \\
\hline $39 S$ & WZ & 1 & $\mathbf{S}$ & Gold Run (west branch) & Well 22BR & Well 22BR \\
\hline $41 S$ & WZ & 1 & L. & Gold Run (west branch) & Well 20BR & Well BRP2 \\
\hline 11MW1 & WZ & 1 & $S$ & Gold Run (west branch) & Gold Run (west branch) & Gold Run (west branch) \\
\hline 12MW1 & WZ & 1 & $\mathrm{~S}$ & Gold Run (west branch) & Gold Run (west branch) & Gold Run (west br?nch) \\
\hline 35MW 1 & WZ & 1 & $\mathrm{~L}$ & Villa Victoria Brook & Well 15BR & Well 15BR \\
\hline BRP1 & $\mathrm{L} 20$ & 22 & $\mathrm{~L}$ & Gold Run (west branch) & Well 15BR & Well 15BR \\
\hline BRP2 & L17 & 25 & $\mathrm{~L}$ & Gold Run (west branch) & Well 20BR & Well BRP2 \\
\hline BRP3 & L19 & 23 & $\mathrm{~L}$ & Gold Run (west branch) & Well 15BR & Well 15BR \\
\hline 2BR & $\mathrm{L} 20$ & 22 & $\mathrm{~L}$ & Delaware River & Well 48BR & Well 48BR \\
\hline 4BR & L17 & 25 & $\mathrm{~L}$ & Gold Run (west branch) & Well 20BR & Well BRP2 \\
\hline $5 \mathrm{BR}$ & L15 & 27 & $L$ & Gold Run (west branch) & Well 20BR & Well BRP2 \\
\hline 7BR & L18 & 24 & $\mathrm{~L}$ & Gold Run (west branch) & Well 15BR & Well 15BR \\
\hline 8BR & $\mathrm{L} 22$ & 20 & $\mathrm{~L}$ & Gold Run (west branch) & Well 15BR & Well 15BR \\
\hline 9BR & $\mathrm{L} 23$ & 19 & $\mathrm{~L}$ & Villa Victoria Brook & Well 15BR & Well 15BR \\
\hline $11 \mathrm{BR}$ & L21 & 21 & $\mathrm{~L}$ & $\begin{array}{l}\text { West Branch Sha- } \\
\text { bakunk Creek }\end{array}$ & Well 48BR & Well 48BR \\
\hline
\end{tabular}


Table 10. Simulated discharge points of ground water at and near contaminated wells at the Naval Air Warfare Center (NAWC), West Trenton, New Jersey--Continued

[WZ, weathered zone; gal/min, gallons per minute; L, Lockatong; S, Stockton; --, no data)]

\begin{tabular}{|c|c|c|c|c|c|c|}
\hline \multirow[b]{2}{*}{ Well name } & \multirow[b]{2}{*}{$\begin{array}{c}\text { Primary } \\
\text { bedding } \\
\text { unit } \\
\text { (Lacombe, } \\
\text { 2000b) }\end{array}$} & \multirow[b]{2}{*}{$\begin{array}{c}\text { Model } \\
\text { layer } \\
\text { num- } \\
\text { ber }\end{array}$} & \multirow[b]{2}{*}{$\begin{array}{c}\text { Bed- } \\
\text { rock } \\
\text { for- } \\
\text { mat- } \\
\text { ion }\end{array}$} & \multicolumn{3}{|c|}{ Stream or pumped well where water discharges from ground-water sy:tem } \\
\hline & & & & $\begin{array}{c}\text { Simulation } 1 \\
\text { (no pumpage at the } \\
\text { NAWC) }\end{array}$ & $\begin{array}{c}\text { Simulation 2, } \\
\text { recovery-well } \\
\text { network I } \\
\text { (well 15BR pumped at } \\
15 \mathrm{gal} / \mathrm{min} ; 20 \mathrm{BR} \text { at } 8.5 \\
\mathrm{gal} / \mathrm{min} ; 22 \mathrm{BR} \text { at } 4.4 \\
\mathrm{gal} / \mathrm{min} ; 41 \mathrm{BR} \text { at } 10 \\
\mathrm{gal} / \mathrm{min} ; 45 \mathrm{BR} \text { at } 5.1 \\
\mathrm{gal} / \mathrm{min} ; 48 \mathrm{BR} \text { at } 13.5 \\
\mathrm{gal} / \mathrm{min} \text { ) }\end{array}$ & $\begin{array}{c}\text { Simulation 3, } \\
\text { recovery-well } \\
\text { network II } \\
\text { (well 15BR pumpe } 1 \text { at } \\
15 \mathrm{gal} / \mathrm{min} \text {; 20BR at } 8.5 \\
\mathrm{gal} / \mathrm{min} ; 22 \mathrm{BR} \text { at } 4.4 \\
\mathrm{gal} / \mathrm{min} ; 45 \mathrm{BR} \text { at } 5.1 \\
\mathrm{gal} / \mathrm{min} ; 48 \mathrm{BR} \text { at } 13.5 \\
\mathrm{gal} / \mathrm{min} \text {; BRP2 at } 10 \\
\mathrm{gal} / \mathrm{min} \text { ) } \\
\end{array}$ \\
\hline 12BR & $\mathrm{L} 22$ & 20 & $\mathrm{~L}$ & Delaware River & Well 48BR & Well 48BR \\
\hline $15 \mathrm{BR}$ & L19 & 23 & $\mathrm{~L}$ & Gold Run (west branch) & Well 15BR & Well 15BR \\
\hline 16BR & L19 & 23 & $\mathrm{~L}$ & Gold Run (west branch) & Well 41BR & Well BRP2 \\
\hline 17BR & L19 & 23 & $\mathrm{~L}$ & Gold Run (west branch) & Well 15BR & Well BRP2 \\
\hline 20BR & L17 & 25 & $\mathrm{~L}$ & Gold Run (west branch) & Well 20BR & Well 20BR \\
\hline 21BR & L23 & 19 & L & Villa Victoria Brook & Well 45BR & Well 45BR \\
\hline 22BR & S13 & 29 & $s$ & Gold Run (west branch) & Well 22BR & Well 22BR \\
\hline $23 \mathrm{BR}$ & L16 & 26 & $\mathrm{~L}$ & Gold Run (west branch) & Well 20BR & Well BRP2 \\
\hline 24BR & L16 & 26 & $\mathrm{~L}$ & Gold Run (west branch) & Well 20BR & Well BRP2 \\
\hline $25 \mathrm{BR}$ & L18 & 24 & $\mathrm{~L}$ & Gold Run (west branch) & Well 15BR & Well 15BR \\
\hline 27BR & L13 & 29 & $\mathrm{~L}$ & Gold Run (west branch) & Well 22BR & Well 22BR \\
\hline 29BR & L21 & 21 & $\mathrm{~L}$ & Gold Run (west branch) & Well 15BR & Well 15BR \\
\hline 30BR & L18 & 24 & $\mathrm{~L}$ & Gold Run (west branch) & Well 15BR & Well BRP2 \\
\hline $31 \mathrm{BR}$ & L15 & 27 & $\mathrm{~L}$ & Gold Run (west branch) & Well 48BR & Well 48BR \\
\hline 36BR & L19 & 23 & $\mathrm{~L}$ & Gold Run (west branch) & Well 15BR & Well 15BR \\
\hline 37BR & S11 & 31 & S & Gold Run (west branch) & Gold Run (west branch) & Gold Run (west branch) \\
\hline 38BR & L19 & 23 & $\mathbf{L}$ & Gold Run (west branch) & Well 15BR & Well 15BR \\
\hline $40 \mathrm{BR}$ & L18 & 24 & $\mathrm{~L}$ & Gold Run (west branch) & Well 20BR & Well 20BR \\
\hline $41 B R$ & L19 & 23 & $\mathrm{~L}$ & Gold Run (west branch) & Well 41BR & Well BRP2 \\
\hline $45 \mathrm{BR}$ & L19 & 23 & $\mathrm{~L}$ & Delaware River & Well 45BR & Well 45BR \\
\hline 46BR & L19 & 23 & $\mathrm{~L}$ & Delaware River & Well 45BR & Well 45BR \\
\hline $47 \mathrm{BR}$ & WZ & 1 & $\mathrm{~L}$ & Gold Run (west branch) & Well 15BR & Well BRP2 \\
\hline $48 \mathrm{BR}$ & L17 & 25 & $\mathrm{~L}$ & Gold Run (west branch) & Well 48BR & Well 48BR \\
\hline 49BR & L17 & 25 & $\mathrm{~L}$ & Delaware River & Well 48BR & Well BRP2 \\
\hline $50 \mathrm{BR}$ & L17 & 25 & $\mathrm{~L}$ & $\begin{array}{l}\text { West Branch Sha- } \\
\text { bakunk Creek }\end{array}$ & Well 48BR & Well 48BR \\
\hline 51BR & $\mathrm{L} 20$ & 22 & $\mathbf{L}$ & Delaware River & Well 48BR & Well 48BR \\
\hline 54BR & -- & 30 & $\mathrm{~L}$ & Gold Run (west branch) & Well 22BR & Well 22BR \\
\hline $56 \mathrm{BR}$ & L17 & 25 & $\mathrm{~L}$ & Villa Victoria Brook & Well 15BR & Well 15BR \\
\hline
\end{tabular}




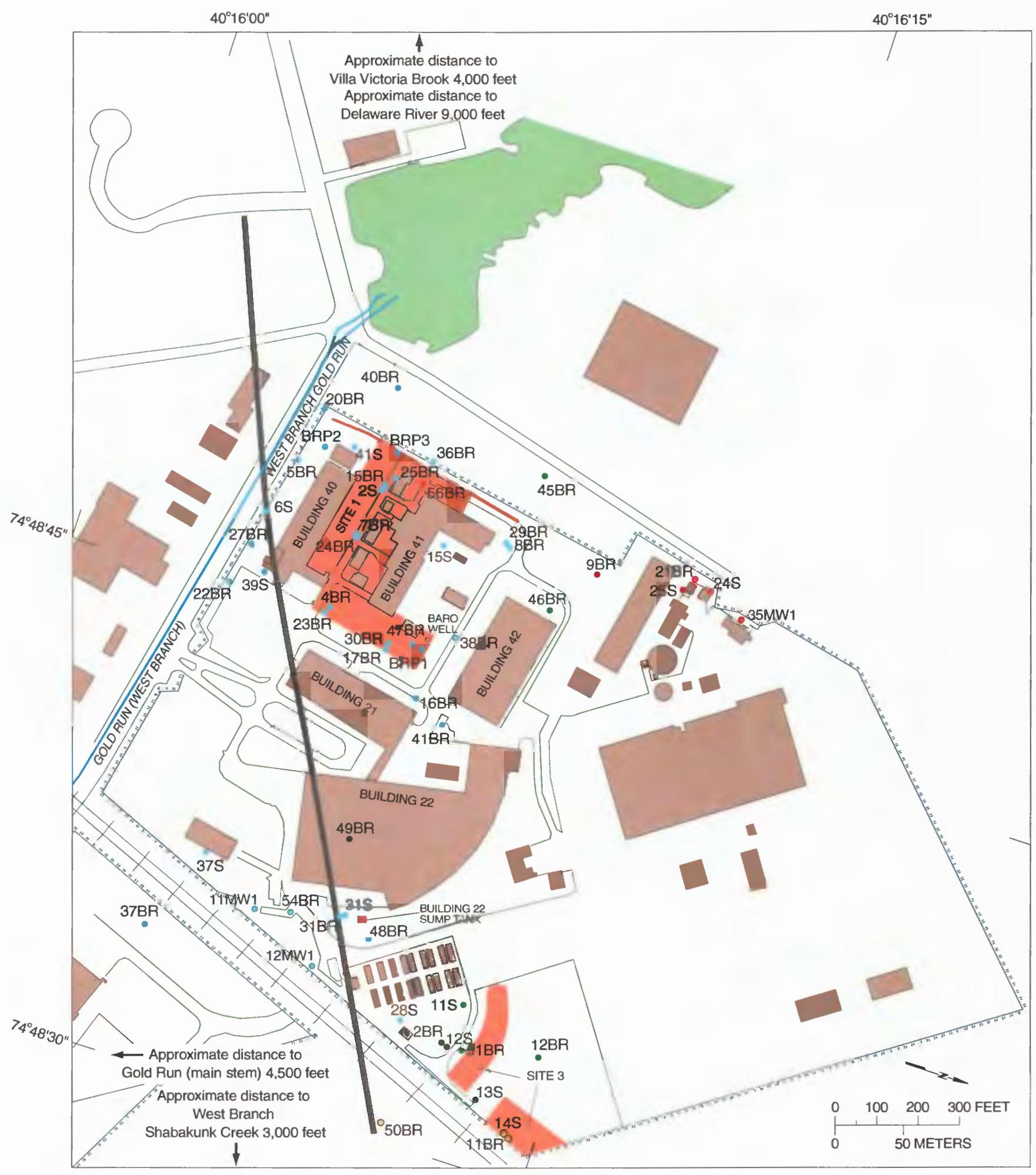

\section{EXPLANATION}

Contaminant source area

Swamp

Building
Fault zone - shows the fault contact at an altitude of 150 feet (approximately land surface)

$25 S^{*}$ Contaminated well and identifier; flow at and near this site simulated to discharge at Villa Victoria Brook

31BR* Contaminated well and identifier; flow at and near this site simulated to discharge at Gold run (West Branch)

12S Contaminated well and idenitifer; flow at and near this site simulated to discharge at Gold run (main stem)

115 Contaminated well and identifier; flow at and near this site simulated to discharge at Delaware River

$11 \mathrm{BR}^{\circ}$ Contaminated well and identifier; flow at and near this site simulated to discharge at West Branch of Shabakunk Creek

Figure 8. Simulated discharge locations of ground water at and near contaminated wells at the Naval Air Warfare Center, West Trenton, New Jersey (no recovery wells simulated as pumping). 
and 12BR) are at the eastern part of the NAWC. Water originating at these locations flows toward the fault in confined layers below the weathered zone, then alongside the fault, and then upward into the river. Water that originates at or near two contaminated wells (12S and 13S) flows in much the same pattern until it reaches the fault, but it then crosses the fault and eventually discharges into the Gold Run main stem.

Ground-water flowpaths in the no-pumping simulation are strongly affected by the hydrogeologic framework. When water from precipitation recharges the ground-water system, it first enters the weathered zone. If the precipitation falls near a stream, the water remains in the weathered zone and discharges to the stream. Precipitation that falls farther from a stream, however, flows through the weathered zone and then into the underlying bedrock, where the fractures are more open and hydraulic conductivity is higher than in the weathered zone. In the bedrock, water probably flows mostly in bedding-plane fractures because these fractures are much more continuous than the near-vertical fractures. Water does move from one bedding-plane fracture to the next, however, through the relatively short vertical fractures. Overall movement of ground water, then, probably is in an elongated stairstep-like pattern, with the near-horizontal (bedding-plane) fractures analogous to long steps and the near-vertical fractures analogous to short risers. In the absence of pumping or other artificial stresses, ground-water flows in this stairstep-like path until it reaches a stream (fig. 9).

The overall direction of flow is nearly parallel to the strike direction of the bedding units (west-northwest and east-southeast). This flow direction results because (1) numerous thin waterbearing units and confining units are present within each bedding unit, and (2) all of the units dip, so that the water-bearing units are extensive in the strike direction but not in the dip direction. Each waterbearing unit terminates updip at the weathered zone and downdip at a depth of about $500 \mathrm{ft}$, where fractures are sparse. Consequently, in each waterbearing unit, flow is constrained in both the updip direction (south-southeast) and the downdip direction (north-northwest), so flow is predominantly to the west-southwest or eastnortheast.

\section{Simulation 2--Recovery-Well Network I}

This simulation represents water levels and flowpaths at times when recovery-well Network I had been pumping for at least 1 month. Under these conditions, ground-water flowpaths near the pumped well are diverted from the ambient strike direction. Under these conditions, one of the recovery wells captures water from areas around 51 of the 55 contaminated wells. The other four contaminated wells (37S, MW11-1, MW12-1, and 37BR) are all on the south side of the fault and more than $650 \mathrm{ft}$ from the only recovery well south of the fault (22BR). Details of the results of this scenario are shown in figure 10 and listed in table 10.

\section{Simulation 3--Recovery-Well Network II}

This simulation represents water levels and flowpaths at times when recovery-well network II had been pumping for at least 1 month. In this network, well BRP2 is pumped instead of well 41BR. In this simulation, water from areas around the same 51 contaminated wells captured by network I is captured by network II although, in many cases, water is captured by a different recovery well. As explained in the earlier section on simulated water budgets, the primary difference between the effects of recovery-well networks I and II is that network II prevents any water from the southwestern part of the NAWC property from flowing into Gold Run. Details of the results of this simulation are shown in figure 10 and listed in table 10. 


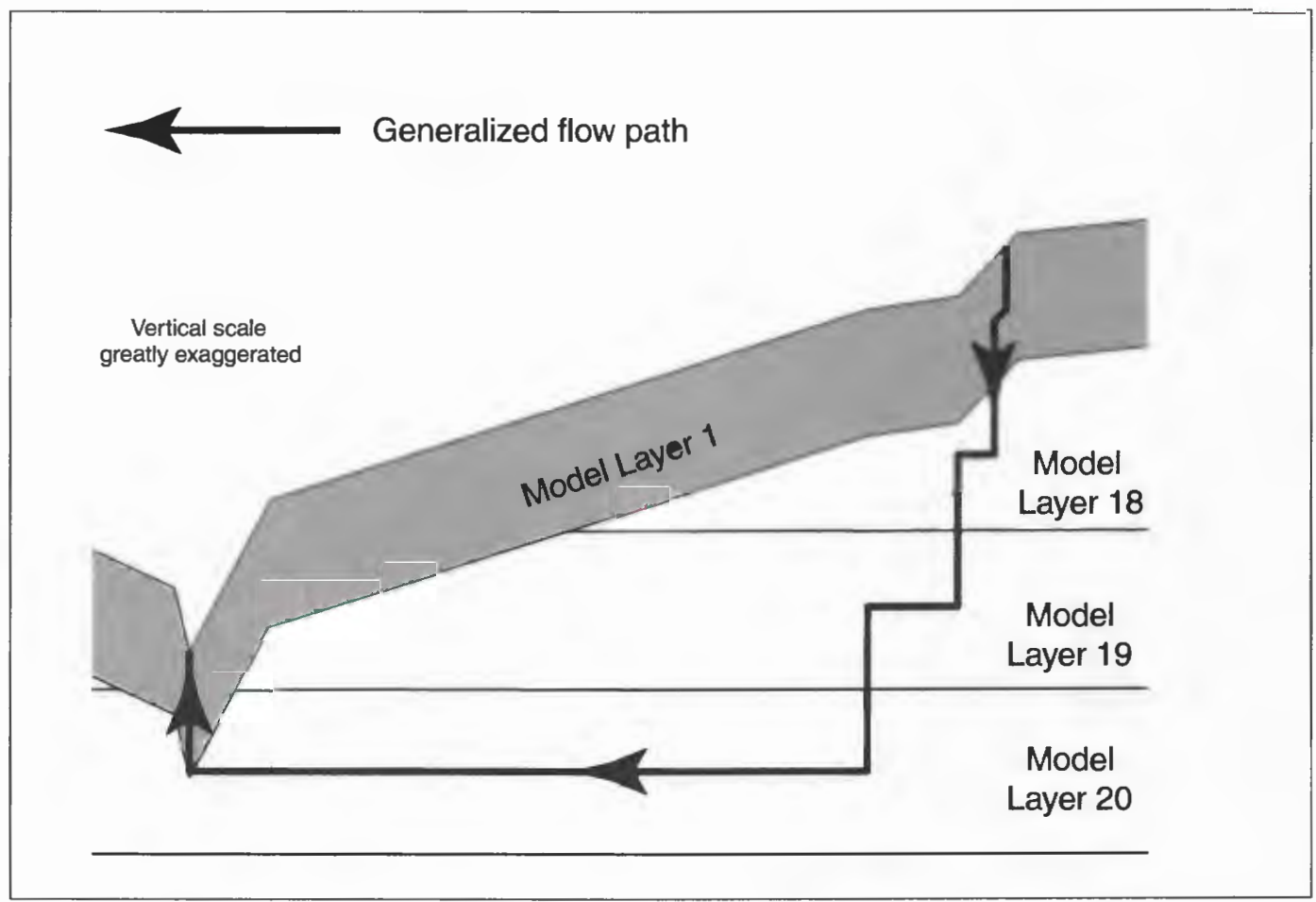

Figure 9. Generalized ground-water-flow path. (Section is oriented paralllel to the strike of bedding.) 


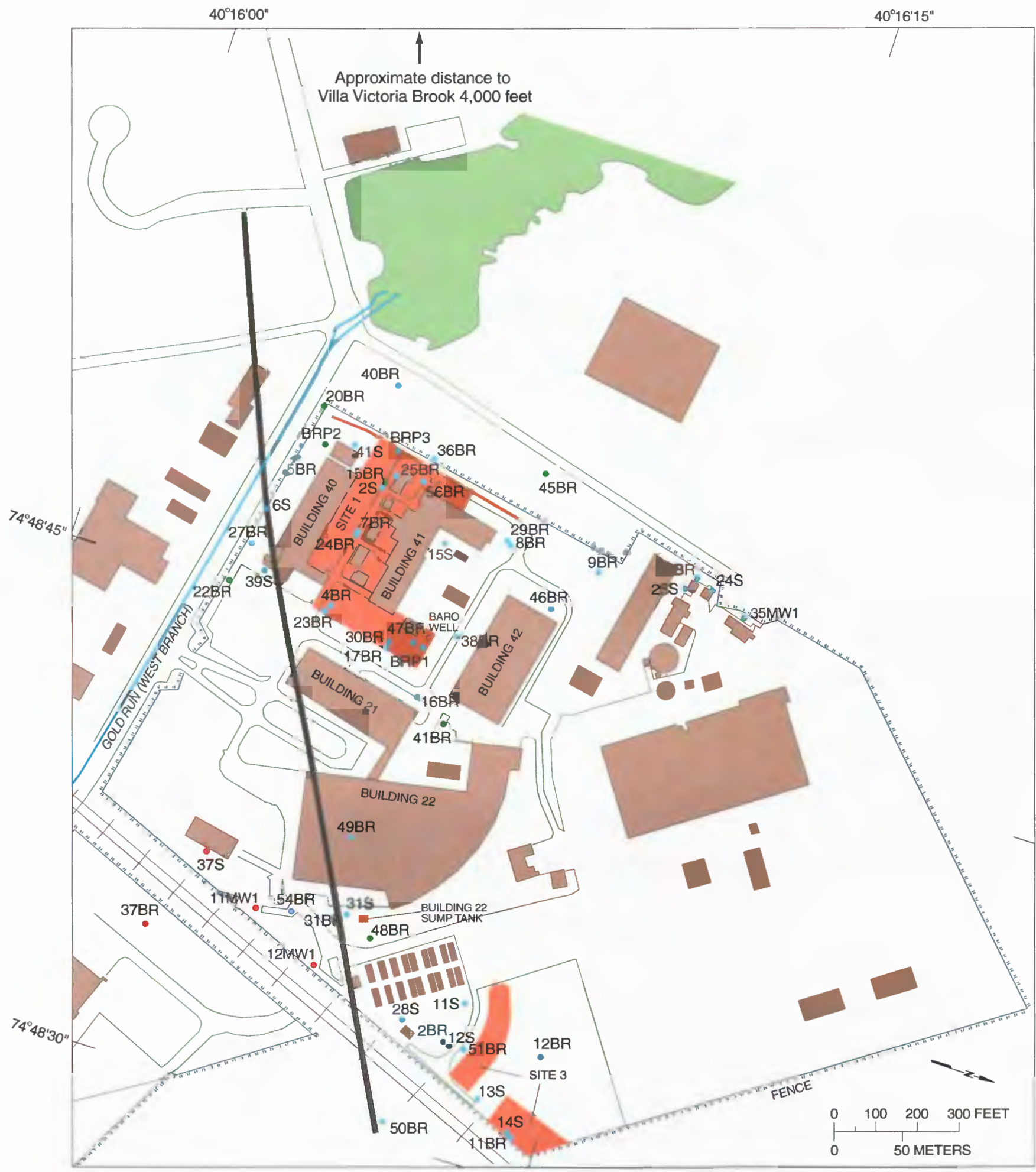

\section{EXPLANATION}

$\begin{array}{lrl}\begin{array}{l}\text { Contaminant } \\ \text { source area }\end{array} & & \begin{array}{l}\text { Fault zone - shows the fault contact at an altitude } \\ \text { of } 150 \text { feet (approximately land surface) }\end{array} \\ \text { Swamp } & 12 \mathrm{MW1} & \begin{array}{l}\text { Contaminated well and identifier; flow at and near this site simulated to discharge at Gold Run (West Branch) } \\ \text { the Naval Air Warfare Center }\end{array} \\ \text { Building } & 11 \mathrm{BR} * & \text { Contaminated well and identifier; flow at and near this site simulated to discharge at recovery well } \\ & 22 \mathrm{BR} & \text { - }\end{array}$

Figure 10. Simulated discharge locations of ground water at and near contaminated wells at the Naval Air Warfare Center, West Trenton, New Jersey (Recovery-well Network I or II in operation). 


\section{SUMMARY AND}

\section{CONCLUSIONS}

Volatile organic compounds, predominantly trichloroethylene (TCE) and its degradation products cis-1,2-dichloroethylene (cisDCE) and vinyl chloride (VC), have been detected in ground water at the Naval Air Warfare Center (NAWC), West Trenton, N.J. (International Technology Corporation, 1994). A study by the U.S. Geological Survey, in cooperation with the U.S. Department of the Navy, was done to determine (1) the directions that contaminated water at the NAWC would move when no pumping occurs at the NAWC and (2) the efficiency of the two alternative recovery-well networks that have been used at the NAWC in containing ground-water contamination on the NAWC property. The primary means of investigation was a digital model of ground-water flow at and near the NAWC. The model area is bounded by natural hydrologic boundaries, including streams, drainage-basin boundaries, and ground-water flowlines parallel to the boundary that don't cross the boundary.

The Lockatong and Stockton Formations form the bedrock in the study area. Both formations consist of interlayered mudstone, siltstone, and sandstone. The rock layers dip north-northwest. Ground water flows mostly through three sets of fractures that are nearly perpendicular to each other. Bedding-plane fractures extend for much longer distances than vertical fractures, and most of the water probably flows in bedding-plane fractures. Vertical fractures carry water from one beddingplane fracture to the next. Near land surface, the rocks are weathered, and fractures are filled with clay and silt from the weathering process. A nearly impermeable fault cuts through the southern part of the NAWC property. The fault is at or near the contact of the Stockton Formation to the south and the Lockatong Formation to the north.

The model used in this study is a finitedifference, three-dimensional, porous-medium representation of the ground-water-flow system. It is composed of 154 rows, 160 columns, and 71 layers. Cells within the NAWC property are 25 to $50 \mathrm{ft}$ wide and 25 to $50 \mathrm{ft}$ long. Elsewhere, the cells are larger, with a maximum size of $500 \mathrm{ft}$ by $6 \Xi ? \mathrm{ft}$ near the model boundaries. Model layer 1 represents the highly weathered part of each dipping bedding unit near land surface. The otl or 70 layers represent the deeper, less weathered part of each dipping bedding unit.

Horizontal hydraulic conductivity was measured using slug testing at 72 wells and estimated at 8 wells on the NAWC property. Tie range of hydraulic conductivity at these well sites was 0.001 to $227 \mathrm{ft} / \mathrm{d}$. The slug-test data indicate that the median hydraulic conductivity in the weathered zone is $2.6 \mathrm{ft} / \mathrm{d}$, whereas the median hydraulic conductivity in wells 26 to $50 \mathrm{ft}$ belc $\mathrm{N}$ land surface is $11.3 \mathrm{ft} / \mathrm{d}$. Conductivity in the weathered zone is lower probably because the fractures are partly filled by clay and silt derived from the weathering process. Deeper than $50 \mathrm{f}$. below land surface, hydraulic conductivity decreases and becomes negligible deeper than $\mathbf{5 0 0}$ $\mathrm{ft}$ below land surface. The decrease in horizontal hydraulic conductivity at depths greater than $57 \mathrm{ft}$ probably is caused by a decrease in the density of interconnected fractures at increasing depths. Ir the calibrated model, the hydraulic conductivity of the weathered zone was set at values ranging from 1.25 to $5.0 \mathrm{ft} / \mathrm{d}$, depending on location. The confined bedding units at depths less than $300 \mathrm{ft}$ below land surface were assigned depth- and location-baset horizontal hydraulic conductivity values ranging from 0.0375 to $11.3 \mathrm{ft} / \mathrm{d}$. The horizontal hydraulic conductivity at all points $300 \mathrm{ft}$ deep or deeper below land surface and all points in the fault zone was set at $0.001 \mathrm{ft} / \mathrm{d}$.

In the model, the vertical hydraulic conductivity of each model cell was set at one-tenth the horizontal hydraulic conductivity. The vertisal hydraulic conductivity of all streambeds in the study area was set at $0.5 \mathrm{ft} / \mathrm{d}$. Because the bed of the Delaware River is thicker than the beds of its tributaries, the conductance of the bed of the river is lower than that of the tributaries. Effective areal recharge rates used in the model are $11 \mathrm{in} / \mathrm{yr}$ in agricultural and undeveloped areas and $5 \mathrm{in} / \mathrm{yr}$ in residential, commercial, and industrial areas. Specific yield of the weathered zone was set at C.15, and the specific storage of the confined layers was set at $4.0 \times 10^{-5}$. 
The steady-state model was calibrated by obtaining acceptably close agreement between measured and simulated ground-water levels at and near the NAWC property and base flow in nearby streams. The transient model was calibrated by obtaining an acceptable agreement between measured and simulated drawdown in wells during three different periods of short-term pumping (72 hours or less) at the NAWC.

Most of the data used in calibrating the model were collected at the NAWC property. Consequently, it would not be appropriate to use this model to obtain detailed simulations of groundwater flow outside of the NAWC area. Another limitation of the model is that only advective movement of contaminants is simulated; dispersion, diffusion, dilution, and biological and chemical degradation of contaminants are not simulated.

To test the effectiveness of the two alternative recovery-well networks in preventing movement of contaminated water to areas beyond the NAWC property, the directions of movement of contaminated water with and without recovery-well networks in operation were estimated by use of the ground-water-flow model. Because the model is based on various necessary simplifying assumptions, the simulated flowpaths and discharge points are approximate. For both simulations, the discharge point of water from the area around each contaminated well at the NAWC was determined. Model results indicate that when no wells at NAWC were pumped, most of the ground water at the
NAWC flowed west-southwest to the reach of Gold Run that is adjacent to and on the NAWC prc nerty. Some contaminated water flowed to Villa Victoria Brook, West Branch Shabakunk Creek, and the Delaware River. In this no-pumping scenaric, ground water flows primarily in the direction of the strike of the bedding units (west-northwest and east-southeast) and probably mostly in fractures parallel to bedding.

Both recovery-well networks that have been used at the NAWC consist of five wells on the north (Lockatong) side of the fault pumping a total of $52.1 \mathrm{gal} / \mathrm{min}$ and one well on the south (Stockton) side of the fault pumping $4.4 \mathrm{gal} / \mathrm{min}$. Network I was used during January 1998 through November 2000. The network was modified in December 2000 because static water-level measurements indicated that contaminated water may have been discharging into the upper 50?-ft reach of Gold Run. In network II, which beg $n$ operating in December 2000, well BRP2 replaced well 41BR because it is closer to Gold Run than well 41BR and more likely to capture water flowing to Gold Run. Water budgets based on simulations representing each of the recovery-well networks indicate that network II prevents any ground water from flowing to the upper $500 \mathrm{ft}$ of Gold Run. Flowpaths computed for each simulation ind'sate that, in both networks, water at and near 51 of the 55 contaminated well sites at the NAWC is cartured by a recovery well. The four well sites from which contaminated water is not captured by a recovery well are on the south side of the fault and more than $650 \mathrm{ft}$ from the nearest recovery well. 


\section{REFERENCES}

Cooper, H.H., Jr., Bredehoeft, J.D., and Papadopoulos, I.S., 1967, Response of a finite-diameter well to an instantaneous charge of water: Water Resources Research, v. 3, no. 1, p. 263-269.

EA Engineering, Science, and Technology, Inc., 1996, Aquifer test report for Naval Air Warfare Center, Trenton (Ewing Township), New Jersey: Berkeley Heights, N.J., v. 1, unpaginated. Harbaugh, A.W., 1990, A computer program for calculating subregional water budgets using results from the U.S. Geological Survey modular three-dimensional finite-difference groundwater flow model: U.S. Geological Survey Open-File Report 90-392, 46 p.

Heath, R.C., 1983, Basic ground-water hydrology: U.S. Geological Survey Water-Supply Paper $2220,84 \mathrm{p}$.

Houghton, H.F., 1990, Hydrogeology of the Early Mesozoic rocks of the Newark Basin, New Jersey, in Kroll, R.L., and Brown, J.O., compilers, Aspects of groundwater in New Jersey, Field guide and proceedings of the seventh annual meeting of the Geological Association of New Jersey: Union, N.J., Geological Association of New Jersey, p. E1-E36.

Hvorslev, M.J., 1951, Time lag and soil permeability in ground-water observations: Corps of Engineers, U.S. Army Waterways Experiment Station Bulletin No. 36, 50 p.

International Technology Corporation, 1994, Remedial investigation report, installation restoration program, Naval Air Warfare Center, Trenton, New Jersey: Contract N624-86-c1041, Project number 529538: Edison, N.J., IT Corporation, $6 \mathrm{v}$.

Kasabach, H.F., 1966, Geology and ground water resources of Hunterdon County, N.J.: New Jersey Bureau of Geology, Bureau of Geology and Topography, Division of Resource Development, Special Report No. 24, 128 p.

Knopman, D.S., 1990, Factors related to the wateryielding potential of rocks in the Piedmont and Valley and Ridge Provinces of Pennsylvania: U.S. Geological Survey Water-Resources Investigations Report 90-4174, 52 p.

Lacombe, P.J., 2000, Hydrogeologic framework, water levels, and trichloroethylene contamina- tion, Naval Air Warfare Center, West Trenton, New Jersey, 1993-97: U.S. Geological Survey Water-Resources Investigations Report 98-4167, 139 p.

Lacombe, P.J., 2001, Water levels and potentiometric surfaces, Naval Air Warfare Center, Trenton, New Jersey, 2000: U.S. Geological Sur'rey Water-Resources Investigations Report 01-4197, $46 \mathrm{p}$.

Lewis-Brown, J.C., and Jacobsen, Eric, 1995, Hydrogeology and ground-water flow, fractured Mesozoic structural-basin rocks, Stony Brook, Beden Brook, and Jacobs Creek drc inage basins, west-central New Jersey: U.S. Geological Survey Water-Resources Investigations Report 94-4147, 83 p.

McDonald, M.G., and Harbaugh, A.W., 1988, A modular three-dimensional finite-differenc? ground-water flow model: U.S. Geological Survey Techniques of Water-Resources Investigations, book 6, chap. A1, 528 p.

National Oceanic and Atmospheric Administration, 2000, Climatological Data, New Jersey, May 2000: Asheville, N.C., v. 105, no. 05, ISSN 0364-5614.

New Jersey Department of Environmental Protection, 1913, New Jersey Atlas Sheet 27, Original Survey 1885 , principal features revised 1913: Trenton, N.J., New Jersey Department of Environmental Protection, 1 sheet.

Pollock, D.W., 1989, Documentation of computer programs to compute and display pathlines using results from the U.S. Geological Sur 'ey modular three-dimensional finite-differenc: ground-water flow model: U.S. Geological Survey Open-File Report 89-381, 188 p.

Rare Earth Envirosciences, Inc., 1992, Environmental Cleanup Responsibility Act proposed ground water cleanup plan for Roller Bearing Company of America, Sullivan Way, West Trenton, Mercer County, New Jersey: ECR A Case \# 85396: Conshohocken, Pa., 170 p.

Rima, D.R., Meisler, Harold, and Longwill, Stanley, 1962, Geology and hydrology of the Stockton Formation in southeastern Pennsylvania A study of the effect of lithology upon the yield of wells: Pennsylvania Geological Survey, Fourth series, Bulletin W14, 46 p. 


\section{REFERENCES--Continued}

Roy F. Weston, Inc., 1989, Pumping test and aquifer analysis, underground storage tank investigation prepared for General Motors Corporation Fisher Guide Division, Trenton, New Jersey:

Bannockburn, Ill., Roy F. Weston, Inc., 38 p.

Vecchioli, John, and Palmer, M.M., 1962, Groundwater resources of Mercer County, New Jersey; New Jersey Department of Conservation and Economic Development Special Report 10, $71 \mathrm{p}$. 